\title{
A dimension-breaking phenomenon for water waves with weak surface tension
}

\author{
M. D. Groves* \\ S. M. Sun ${ }^{\dagger}$ \\ E. Wahlén
}

\begin{abstract}
It is well known that the water-wave problem with weak surface tension has smallamplitude line solitary-wave solutions which to leading order are described by the nonlinear Schrödinger equation. The present paper contains an existence theory for three-dimensional periodically modulated solitary-wave solutions which have a solitary-wave profile in the direction of propagation and are periodic in the transverse direction; they emanate from the line solitary waves in a dimension-breaking bifurcation. In addition, it is shown that the line solitary waves are linearly unstable to long-wavelength transverse perturbations. The key to these results is a formulation of the water wave problem as an evolutionary system in which the transverse horizontal variable plays the role of time, a careful study of the purely imaginary spectrum of the operator obtained by linearising the evolutionary system at a line solitary wave, and an application of an infinite-dimensional version of the classical Lyapunov centre theorem.
\end{abstract}

\section{Introduction}

In this article we consider the three-dimensional irrotational flow of a perfect fluid of unit density subject to the forces of gravity and surface tension. The fluid is bounded below by a rigid horizontal bottom $\{y=0\}$ and above by a free surface $\{y=h+\eta(x, z, t)\}$. Our primary interest is in travelling waves moving without change of shape and with constant speed $c>0$ from left to right in the $x$-direction. In a moving frame of reference in which such waves are stationary the equations of motion are Laplace's equation

$$
\phi_{x x}+\phi_{y y}+\phi_{z z}=0, \quad 0<y<1+\eta,
$$

for the Eulerian velocity potential $\phi$ describing the flow, with boundary conditions

$$
\begin{aligned}
\phi_{y} & =0, & y & =0, \\
\phi_{y} & =\eta_{t}-\eta_{x}+\eta_{x} \phi_{x}+\eta_{z} \phi_{z}, & & y=1+\eta,
\end{aligned}
$$

*Fachrichtung 6.1 - Mathematik, Universität des Saarlandes, Postfach 151150, 66041 Saarbrücken, Germany; Department of Mathematical Sciences, Loughborough University, Loughborough, LE11 3TU, UK

${ }^{\dagger}$ Department of Mathematics, Virginia Polytechnic Institute and State University, Blacksburg, VA 24061, USA

$\ddagger$ Centre for Mathematical Sciences, Lund University, PO Box 118, 22100 Lund, Sweden 
and

$$
\phi_{t}-\phi_{x}+\frac{1}{2}\left(\phi_{x}^{2}+\phi_{y}^{2}+\phi_{z}^{2}\right)+\alpha \eta-\beta\left[\frac{\eta_{x}}{\sqrt{1+\eta_{x}^{2}+\eta_{z}^{2}}}\right]_{x}-\beta\left[\frac{\eta_{z}}{\sqrt{1+\eta_{x}^{2}+\eta_{z}^{2}}}\right]_{z}=0, \quad y=1+\eta .
$$

Here we have introduced dimensionless variables, choosing $h$ as length scale and $h / c$ as time scale; the parameters $\alpha$ and $\beta$ are defined in terms of the Froude and Bond numbers $F=c / \sqrt{g h}$ and $\tau=\sigma / g h^{2}$ by the formulae $\alpha=1 / F^{2}$ and $\beta=\tau / F^{2}$, in which $g$ is the acceleration due to gravity and $\sigma$ is the coefficient of surface tension.

Before describing the present contribution, let us briefly review the classical weakly nonlinear theory with weak surface tension (that is, $0<\tau<1 / 3$ ) for stationary wave packets consisting of a slowly varying envelope modulating a periodic wave train. Figure 1 shows the linear dispersion relation for a sinusoidal travelling wave train with wave number $\mu$; for each fixed value $\tau_{0} \in(0,1 / 3)$ of $\tau$ the dispersion curve has a unique minimum at $\left(\mu, \alpha^{-1}\right)=\left(\mu_{0}, \alpha_{0}^{-1}\right)$. (The relationship between $\alpha_{0}, \beta_{0}=\tau_{0} \alpha_{0}$ and $\mu_{0}$ can be expressed in the form

$$
\alpha_{0}=\frac{\mu_{0}^{2}}{2} \operatorname{cosech}^{2} \mu_{0}+\frac{\mu_{0}}{2} \operatorname{coth} \mu_{0}, \quad \beta_{0}=-\frac{1}{2} \operatorname{cosech}^{2} \mu_{0}+\frac{1}{2 \mu_{0}} \operatorname{coth} \mu_{0},
$$

which defines a curve in the $(\alpha, \beta)$-plane parameterised by $\mu \in(0, \infty)$.) Substituting

$$
\alpha=\alpha_{0}+\varepsilon^{2}, \quad \beta=\beta_{0}, \quad \eta(x, z)=\frac{1}{2} \varepsilon \zeta(\varepsilon x, \varepsilon z) \mathrm{e}^{\mathrm{i} \mu_{0} x}+\frac{1}{2} \varepsilon \overline{\zeta(\varepsilon x, \varepsilon z)} \mathrm{e}^{-\mathrm{i} \mu_{0} x}
$$

into equations (1)-(4), one finds that to leading order $\zeta$ satisfies the elliptic-elliptic DaveyStewartson system

$$
\begin{aligned}
\zeta-A_{1} \zeta_{X X}-A_{2} \zeta_{Z Z}-A_{3}|\zeta|^{2} \zeta+4 A_{4} \zeta \psi_{X} & =0 \\
-\left(1-\alpha_{0}^{-1}\right) \psi_{X X}-\psi_{Z Z}-A_{4}\left(|\zeta|^{2}\right)_{X} & =0
\end{aligned}
$$

where $X=\varepsilon x, Z=\varepsilon z$ (see Djordjevic \& Redekopp [8], Ablowitz \& Segur [1], noting the misprint in equation (2.24d), and Sulem \& Sulem [27, \$11.1.1]); formulae for the coefficients $A_{1}, A_{3}$ and $A_{4}$ are given in the Appendix, while $A_{2}=\mu_{0}^{-1} \operatorname{coth} \mu_{0}$. In the special case of $z$-independent waves these equations reduce to the cubic nonlinear Schrödinger equation

$$
\zeta-A_{1} \partial_{X}^{2} \zeta-A_{5}|\zeta|^{2} \zeta=0
$$

where $A_{5}=A_{3}+4\left(1-\alpha_{0}^{-1}\right)^{-1} A_{4}^{2}$.

The water-wave problem (1)-(4) with weak surface tension has two families of $z$-independent, small-amplitude, symmetric solitary-wave solutions $\left(\eta_{\varepsilon}^{\star}, \phi_{\varepsilon}^{\star}\right)$. We refer to these waves, which were discovered by Iooss and Kirchgässner [19] (see also Dias \& Iooss [7] and Iooss \& Pérouème [20]), as line solitary waves; they are stationary solutions of (1)-(4) with

$$
\alpha=\alpha_{0}+\varepsilon^{2}, \quad \beta=\beta_{0}, \quad \eta_{\varepsilon}^{\star}(x)=\varepsilon \zeta^{\star}(\varepsilon x) \cos \left(\mu_{0} x\right)+O\left(\varepsilon^{2}\right),
$$

where the function

$$
\zeta^{\star}(X)= \pm\left(\frac{2}{A_{5}}\right)^{1 / 2} \operatorname{sech}\left(\frac{X}{A_{1}^{1 / 2}}\right)
$$




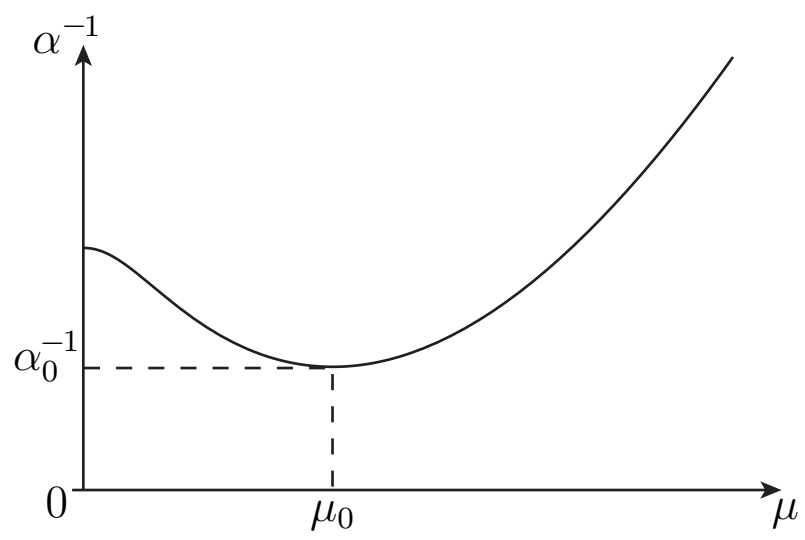

Figure 1: The linear dispersion relation for a sinusoidal travelling wave train with wave number $\mu$ and $\tau=\tau_{0}$, where $\tau_{0} \in(0,1 / 3)$.

is a symmetric solution of the cubic nonlinear Schrödinger equation (and of course a symmetric line solitary-wave solution of the Davey-Stewartson system). The line solitary waves are wave packets consisting of a decaying envelope which modulates an underlying periodic wave train with wave number $\mu_{0}$ (see Figure 2).

In this paper we again consider weak surface tension $(0<\tau<1 / 3)$ and prove the existence of three-dimensional periodically modulated solitary waves which have a solitary-wave profile in the $x$-direction and are periodic in the transverse direction $z$. These waves emanate from the Iooss-Kirchgässner line solitary waves in a dimension-breaking bifurcation - a phenomenon in which a spatially inhomogeneous solution of a partial differential equation emerges from a solution which is homogeneous in one or more spatial dimensions (Haragus \& Kirchgässner [17]) — and are sketched in Figure 2, Periodically modulated solitary waves have also been computed numerically, albeit for water of infinite depth, by Milewski \& Zhang [24].

Our existence proof is based upon spatial dynamics, a framework for studying stationary boundary-value problems by treating them as evolution equations in which an unbounded spatial variable plays the role of time. The method was pioneered by Kirchgässner [22] and used extensively in the study of two-dimensional travelling water waves (see e.g. Groves [11] and the references therein). It was extended to the three-dimensional water-wave setting by Groves \& Mielke [15] and has been used to construct a wide variety of three-dimensional gravity-capillary waves (see Groves \& Haragus [12]). We use the method by formulating the hydrodynamic problem for travelling waves as a reversible evolutionary equation

$$
u_{z}=f(u) \text {, }
$$

where $u$ belongs to a space $\mathcal{X}$ of functions which converge to zero as $x \rightarrow \pm \infty$ and $f: \mathcal{D}(f) \subseteq$ $\mathcal{X} \rightarrow \mathcal{X}$ is an unbounded, densely defined vector field. Observe that the nonzero equilibria of equation (5) are precisely the line solitary-wave solutions of the water-wave problem; of particular interest in this respect are the equilibria $u_{\varepsilon}^{\star}$ corresponding to the Iooss-Kirchgässner line solitary waves. A solution to equation (5) of the form

$$
u(x, z)=u_{\varepsilon}^{\star}(x)+u^{\prime}(x, z),
$$

where $u^{\prime}$ has small amplitude and is periodic in $z$, corresponds to a periodically modulated solitary wave which is generated by a dimension-breaking bifurcation from a Iooss-Kirchgässner 

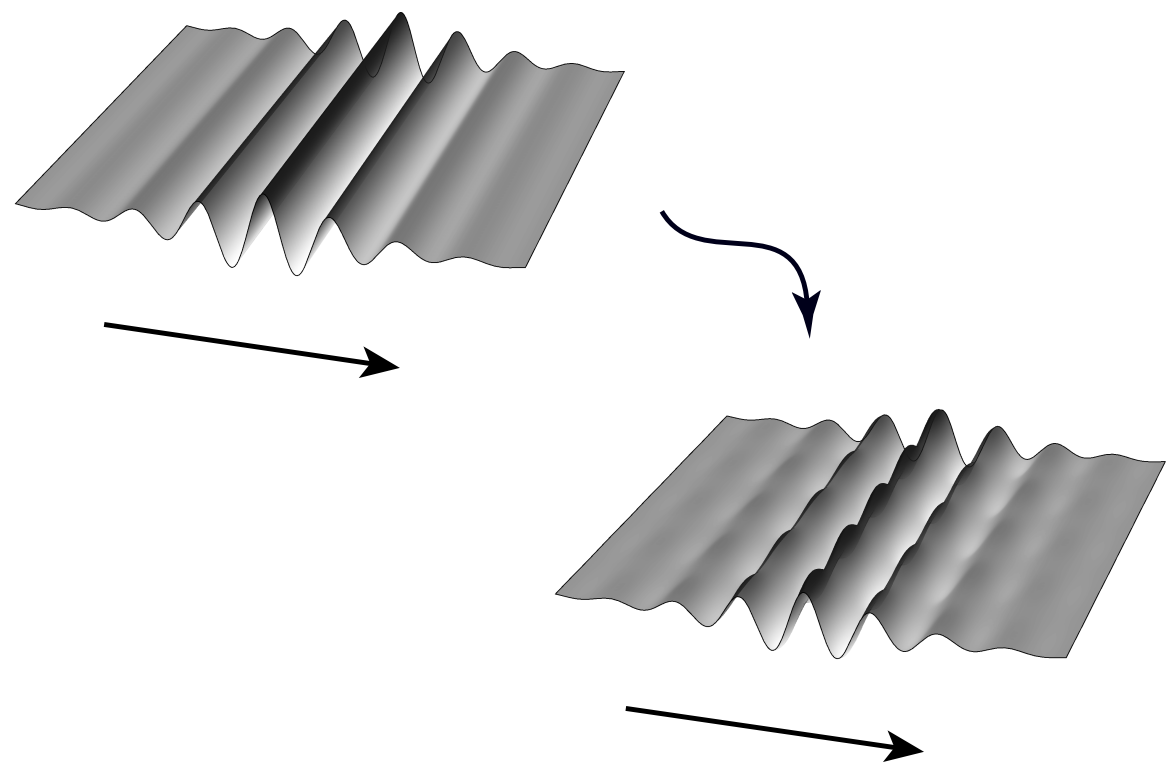

Figure 2: The periodically modulated solitary wave on the right emerges from the line solitary wave on the left in a dimension breaking bifurcation.

line solitary wave. Accordingly, we construct a family of small-amplitude periodic solutions to the equation

$$
u_{z}^{\prime}=f\left(u_{\varepsilon}^{\star}+u^{\prime}\right)
$$

for each sufficiently small value of $\varepsilon$. Explicit formulae for equations (5) and (7) (together with the definitions of suitable function spaces in which to study them) are presented in Section 2.1 below.

Small-amplitude periodic solutions of reversible evolutionary equations are classically found using the Lyapunov centre theorem, which asserts the existence of a family of these solutions with frequency near $\omega_{0}$ provided that $\pm \mathrm{i} \omega_{0}$ are nonresonant imaginary eigenvalues of the corresponding linearised system. The theorem remains true for infinite-dimensional systems with finite-dimensional linear central subspaces under additional hypotheses on the decay rate of the resolvent operator along the imaginary axis (Devaney [6]). In the present setting the central subspace is however infinite-dimensional due to the presence of essential spectrum at the origin (a feature typical of spatial dynamics formulations for problems in unbounded domains). It was pointed out by Iooss [18] that this difficulty does not automatically rule out an application of the reversible Lyapunov centre theorem. Denoting the linear and nonlinear parts of the reversible vector field in question by respectively $L$ and $N$, one finds upon examining the proof of the Lyapunov centre theorem that it is not required that $L$ is invertible on the whole of phase space, rather merely on the range of $N$ ('the Iooss condition at the origin'). We thus arrive at the following generalisation of the reversible Lyapunov centre theorem, which we refer to as the Lyapunov-Iooss theorem (see also Bagri \& Groves [3] for an application to doubly periodic surface waves). 
Theorem 1.1 Consider the differential equation

$$
v_{\tau}=L(v)+N(v),
$$

in which $v(\tau)$ belongs to a Banach space $\mathcal{X}$. Suppose that $\mathcal{Y}, \mathcal{Z}$ are further Banach spaces with the properties that

(i) $\mathcal{Z}$ is continuously embedded in $\mathcal{Y}$ and continuously and densely embedded in $\mathcal{X}$,

(ii) $L: \mathcal{Z} \subseteq \mathcal{X} \rightarrow \mathcal{X}$ is a closed linear operator,

(iii) there is an open neighbourhood $\mathcal{U}$ of the origin in $\mathcal{Y}$ such that $L \in \mathcal{L}(\mathcal{Y}, \mathcal{X})$ and $N \in C_{\mathrm{b}, \mathrm{u}}^{3}(\mathcal{U}, \mathcal{X})$ (and hence $N \in C_{\mathrm{b}, \mathrm{u}}^{3}(\mathcal{U} \cap \mathcal{Z}, \mathcal{X})$ ) with $N(0)=0, \mathrm{~d} N[0]=0$.

Suppose further that

(iv) equation (8) is reversible: there exists an involution $S \in \mathcal{L}(\mathcal{X}, \mathcal{X})$ with $S L v=-L S v$ and $S N(v)=-N(S v)$ for all $v \in \mathcal{U}$,

and that the following spectral hypotheses are satisfied.

(v) $\pm \mathrm{i} \omega_{0}$ are nonzero simple eigenvalues of $L$;

(vi) in $\omega_{0} \in \rho(L)$ for $n \in \mathbb{Z} \backslash\{-1,0,1\}$;

(vii) $\left\|\left(L-\mathrm{i} n \omega_{0} I\right)^{-1}\right\|_{\mathcal{X} \rightarrow \mathcal{X}}=o(1)$ and $\left\|\left(L-\mathrm{i} n \omega_{0} I\right)^{-1}\right\|_{\mathcal{X} \rightarrow \mathcal{Z}}=O(1)$ as $n \rightarrow \infty$;

(viii) For each $v^{\dagger} \in \mathcal{U}$ the equation

$$
L v=-N\left(v^{\dagger}\right)
$$

has a unique solution $v \in \mathcal{Y}$ and the mapping $v^{\dagger} \mapsto u$ belongs to $C_{\mathrm{b}, \mathrm{u}}^{3}(\mathcal{U}, \mathcal{Y})$.

Under these hypotheses there exist an open neighbourhood $\mathcal{N}$ of the origin in $\mathbb{R}$ and a continuously differentiable branch $\{(v(s), \omega(s))\}_{s \in S}$ of reversible, $2 \pi / \omega(s)$-periodic solutions in $C_{\mathrm{per}}^{1}(\mathbb{R}, \mathcal{Y} \oplus \mathcal{X}) \cap C_{\mathrm{per}}(\mathbb{R}, \mathcal{Y} \oplus \mathcal{Z})$ to $(8)$ with amplitude $O(|s|)$. Here the direct sum refers to the decomposition of a function into its mode 0 and higher-order Fourier components, the subscript 'per' indicates a $2 \pi / \omega(s)$-periodic function and $\omega(s)=\omega_{0}+O\left(|s|^{2}\right)$.

Our main task is now to study the purely imaginary spectrum of the linearisation $L$ of the vector field on the right-hand side of (7) (see Sections 3 and 4). Motivated by the weakly nonlinear scaling for modulated wave packets, we use the decomposition $\eta=\eta_{1}+\eta_{2}$, where the supports of the Fourier transforms of $\eta_{1}$ and $\eta_{2}$ lie respectively in $S=\left[-\mu_{0}-\delta,-\mu_{0}+\delta\right] \cup\left[\mu_{0}-\delta, \mu_{0}+\delta\right]$ and $\mathbb{R} \backslash S$, and write

$$
\eta_{1}(x)=\frac{1}{2} \varepsilon \zeta(\varepsilon x) \mathrm{e}^{\mathrm{i} \mu_{0} x}+\frac{1}{2} \varepsilon \overline{\zeta(\varepsilon x)} \mathrm{e}^{-\mathrm{i} \mu_{0} x} .
$$

We find, after a lengthy calculation, that the pair $\pm \mathrm{i} \varepsilon k, k>0$, are eigenvalues of $L$ if and only if the operator

$$
\begin{aligned}
& \mathcal{B}_{\varepsilon, k}(\zeta, \psi):= \\
& \left(\begin{array}{c}
A_{2}^{-1} \zeta-A_{2}^{-1} A_{1} \zeta_{x x}+k^{2} \zeta-A_{2}^{-1}\left(A_{3}+A_{5}\right) \zeta^{\star 2} \zeta-A_{2}^{-1} A_{3} \zeta^{\star 2} \bar{\zeta}+4 A_{2}^{-1} A_{4} \zeta^{\star} \psi_{x}-\mathcal{R}_{\varepsilon, k}(\zeta) \\
-\left(1-\alpha_{0}^{-1}\right) \psi_{x x}+k^{2} \psi-2 A_{4} \operatorname{Re}\left(\zeta^{\star} \zeta\right)_{x}
\end{array}\right)
\end{aligned}
$$


has a zero eigenvalue, where $\mathcal{R}_{\varepsilon, k}(\zeta)$ is a remainder term which is $O(\varepsilon)$ in an appropriate sense; note that $\mathcal{B}_{0, k}(\zeta, \psi)$ is precisely the operator obtained by linearising the Davey-Stewartson system at its line solitary wave and taking the Fourier transform with respect to $z$. A comparison with well-studied operators in mathematical physics shows that $\mathcal{B}_{0,0}=\mathcal{B}_{0, k}-k^{2} I$ has precisely one simple negative eigenvalue $-k_{0}^{2}$, so that $\mathcal{B}_{0, k}$ has a simple zero eigenvalue precisely when $k=k_{0}$, and we deduce from a spectral perturbation argument that $\mathcal{B}_{\varepsilon, k}$ is invertible for $k \in$ $\left[k_{\text {min }}, \infty\right) \backslash\left\{k_{\varepsilon}\right\}$, where $k_{\text {min }}$ is a fixed small, positive number and $\left|k_{0}-k_{\varepsilon}\right|=O(\varepsilon)$, while $\mathcal{B}_{\varepsilon, k_{\varepsilon}}$ has a simple zero eigenvalue; the corresponding eigenvector is invariant under the symmetry $\tilde{R}:(\zeta(x), \psi(x)) \mapsto(\overline{\zeta(-x)},-\psi(-x))$, thus corresponding to a linear water wave whose surface profile is symmetric in $x$.

A similar argument is used to verify the Iooss condition at the origin. Applying the above reduction to the equation

$$
L v=-N\left(v^{\dagger}\right)
$$

where $N$ is the nonlinear part of the vector field on the right-hand side of (7), we obtain the reduced equation

$$
\mathcal{C}_{\varepsilon}(\zeta)=\zeta^{\dagger}
$$

where $\mathcal{C}_{0}$ is the operator obtained by linearising the cubic nonlinear Schrödinger equation at its solitary wave and $\mathcal{C}_{\varepsilon}-\mathcal{C}_{0}$ is $O(\varepsilon)$ in an appropriate sense. The operator $\mathcal{C}_{0}$ is invertible in the class of functions which are invariant under the symmetry $\zeta(x) \mapsto \overline{\zeta(-x)}$, and it follows that the reduced equation is solvable in this class. We also show that $\left.\mathcal{B}_{\varepsilon, k}\right|_{\mathrm{Fix} \tilde{R}}$ has no zero eigenvalue in the interval $\left(0, k_{\min }\right)$. Working in function spaces corresponding to symmetric surface waves, we therefore obtain the following existence result for periodically modulated solitary waves from Theorem 1.1.

Theorem 1.2 There exist an open neighbourhood $\mathcal{N}_{\epsilon}$ of the origin in $\mathbb{R}$ and a family of periodically modulated solitary waves $\left\{\left(\eta_{s}(x, z), \phi_{s}(x, y, z)\right)\right\}_{s \in \mathcal{N}_{\epsilon}}$ which emerges from the IoossKirchgässner line solitary wave in a dimension-breaking bifurcation. Here

$$
\eta_{s}(x, z)=\eta_{\varepsilon}^{\star}(x)+\eta_{s}^{\star}(x, z),
$$

in which $\eta_{s}^{\star}(\cdot, \cdot)$ has amplitude $O(|s|)$ and is even in both arguments and periodic in its second with frequency $\varepsilon k_{\varepsilon}+O\left(|s|^{2}\right)$; the positive number $k_{\varepsilon}$ satisfies $\left|k_{\varepsilon}-k_{0}\right|=O(\epsilon)$.

The spectral information necessary for the Lyapunov-Iooss theorem can also be used to study the stability properties of line solitary waves. The spatial dynamics formulation (5) can be generalised to time-dependent waves; the result is an equation of the form

$$
u_{z}=d u_{t}+f(u)
$$

where $d: \mathcal{D}(f) \subset \mathcal{X} \rightarrow \mathcal{X}$ is a linear operator (see Section 2.1). We say that a line solitary wave $u^{\star}$ (that is, a solution of (9) which is independent of $x$ and $t$ ) is 'transversely linearly unstable' if the linear equation

$$
u_{z}=d u_{t}+\mathrm{d} f\left[u^{\star}\right](u)
$$

has a solution of the form $\mathrm{e}^{\sigma t} u_{\sigma}(z)$, where $\operatorname{Re} \sigma>0$ and $u_{\sigma}$ is bounded (see Section 2.1 for a precise definition). The following theorem, which is due to Godey [10], shows that the existence of a pair of simple purely imaginary eigenvalues of $L=\mathrm{d} f\left[u^{\star}\right]$ implies the transverse linear instability of $u^{\star}$. 
Theorem 1.3 Consider the differential equation

$$
v_{\tau}=d_{1} v_{t}+d_{2} v_{t t}+L v
$$

in which $v(\tau, t)$ belongs to a Banach space $\mathcal{X}$. Suppose that $\mathcal{Z}$ is a further Banach space with the properties that

(i) $\mathcal{Z}$ is continuously and densely embedded in $\mathcal{X}$,

(ii) $L: \mathcal{Z} \subseteq \mathcal{X} \rightarrow \mathcal{X}$ and $d_{j}: \mathcal{Z} \subseteq \mathcal{X} \rightarrow \mathcal{X}$ are closed linear operators,

(iii) the equation is reversible: there exists an involution $S \in \mathcal{L}(\mathcal{X}, \mathcal{X})$ with $L S v=-S L v$ and $d_{j} S v=-S d_{j} v$ for all $v \in \mathcal{Z}$,

(iv) L has a pair $\pm \mathrm{i} \omega_{0}$ of isolated purely imaginary eigenvalues with odd algebraic multiplicity.

Under these hypotheses equation (10) has a solution of the form $\mathrm{e}^{\sigma t} v_{\sigma}(\tau)$, where $v_{\sigma} \in C^{1}(\mathbb{R}, \mathcal{X}) \cap$ $C(\mathbb{R}, \mathcal{Z})$ is periodic, for each sufficiently small positive value of $\sigma$; its period tends to $2 \pi / \omega_{0}$ as $\sigma \rightarrow 0$.

This result applies to the Iooss-Kirchgässner line solitary waves and confirms a prediction made by Ablowitz \& Segur [1, p. 704] on the basis of the Davey-Stewartson approximation; note that it is not necessary to restrict to symmetric surface waves to apply Theorem 1.3 .

Theorem 1.4 The Iooss-Kirchgässner line solitary wave $\left(\eta_{\varepsilon}^{\star}, \phi_{\varepsilon}^{\star}\right)$ is transversely linearly unstable with respect to periodic perturbations with sufficiently large period.

The stability properties of line solitary waves with weak surface tension with respect to twodimensional perturbations were studied by Buffoni [4, 5] and Groves \& Wahlén [16]. They proved the conditional energetic stability of the set of minimisers of the energy subject to the constraint of fixed momentum. The precise relation between the set of minimisers and the IoossKirchgässner waves still remains unclear, however.

The case of strong surface tension (that is, $\tau>1 / 3$ ) is rather different. The linear dispersion curve for a sinusoidal travelling wave train has its minimum at wavenumber $\mu=0$ (and $\alpha=1$ ), and the appropriate weakly nonlinear approximation is the Kadomtsev-Petviashvili (KP-I) equation, which reduces to the Korteweg-deVries equation in the special case of $z$-independent waves. This equation admits explicit line and periodically modulated solitary-wave solutions (Tajiri \& Murakami [28]); the line solitary waves are symmetric waves of depression with monotonically and exponentially decaying tails. An existence theory for line solitary waves in the parameter regime $\alpha=1+\varepsilon^{2}$ and $\beta=\beta_{0}$, where $\beta_{0}>1 / 3$, was given by Kirchgässner [23] and Amick \& Kirchgässner [2], while their dimension breaking to periodically modulated solitary waves was established by Groves, Haragus \& Sun [14] (see also Groves, Haragus \& Sun [13] and Pego \& Sun [25] for a discussion of their linear transverse instability and Rousset and Tzvetkov [26] for a proof of their nonlinear instability with respect to three-dimensional perturbations). The present treatment of the weak surface tension case follows the strategy of Groves, Haragus \& Sun for strong surface tension, and we indeed refer to their paper for the derivation of our spatial dynamics formulation and some a priori linear estimates. Our mathematics, however, differs from theirs in several respects: we use simpler function spaces for the spatial dynamics formulation and work directly with the variable $\eta$, an approach which leads to conceptually simpler calculations and illuminates the connection with the weakly nonlinear model more clearly. 


\section{Dimension-breaking phenomena}

\subsection{Spatial dynamics}

In this section we formulate equations (1)-(4) as an evolutionary equation in which the unbounded horizontal direction $z$ plays the role of time. The first step is to use the change of variable

$$
\phi(x, y, z, t)=\Phi\left(x, y^{\prime}, z, t\right), \quad y=y^{\prime}(1+\eta(x, z, t))
$$

to map the variable fluid domain $\{(x, y, z): x, z \in \mathbb{R}, 0<y<1+\eta(x, z, t)\}$ into the fixed strip $\left\{\left(x, y^{\prime}, z\right) \in \mathbb{R} \times(0,1) \times \mathbb{R}\right\}$; we drop the primes for notational convenience. Defining

$$
\begin{aligned}
\omega & =-\int_{0}^{1}\left(\Phi_{z}-\frac{y \eta_{z} \Phi_{y}}{1+\eta}\right) y \Phi_{y} \mathrm{~d} y+\frac{\beta \eta_{z}}{\sqrt{1+\eta_{x}^{2}+\eta_{z}^{2}}}, \\
\xi & =\left(\Phi_{z}-\frac{y \eta_{z} \Phi_{y}}{1+\eta}\right)(1+\eta)
\end{aligned}
$$

one finds that equations (1)-(4) can be formulated as

$$
u_{z}=d u_{t}+f(u)
$$

for $u=(\eta, \omega, \Phi, \xi)$ with boundary conditions

$$
\Phi_{y}=y \eta_{t}+B(u) \quad \text { on } y=0,1 .
$$

Here

$$
d u=\left(0,\left.\Phi\right|_{y=1}, 0,0\right),
$$

$f(u)=\left(f_{1}(u), f_{2}(u), f_{3}(u), f_{4}(u)\right)$ with

$$
\begin{aligned}
f_{1}(u)=W & \left(\frac{1+\eta_{x}^{2}}{\beta^{2}-W^{2}}\right)^{1 / 2}, \\
f_{2}(u)= & \frac{W}{(1+\eta)^{2}}\left(\frac{1+\eta_{x}^{2}}{\beta^{2}-W^{2}}\right)^{1 / 2} \int_{0}^{1} y \Phi_{y} \xi \mathrm{d} y-\left[\eta_{x}\left(\frac{\beta^{2}-W^{2}}{1+\eta_{x}^{2}}\right)^{1 / 2}\right]_{x}+\alpha \eta-\left.\Phi_{x}\right|_{y=1} \\
& +\int_{0}^{1}\left\{\frac{\xi^{2}-\Phi_{y}^{2}}{2(1+\eta)^{2}}+\frac{1}{2}\left(\Phi_{x}-\frac{y \eta_{x} \Phi_{y}}{1+\eta}\right)^{2}+\left[\left(\Phi_{x}-\frac{y \eta_{x} \Phi_{y}}{1+\eta}\right) y \Phi_{y}\right]_{x}\right. \\
f_{3}(u)= & \left.\left.\frac{\xi}{1+\eta}+\frac{W y \Phi_{y}}{1+\eta}\left(\frac{1+\Phi_{x}}{\beta^{2}-W^{2}}\right)^{1 / 2}, \frac{y \eta_{x} \Phi_{y}}{1+\eta}\right) \frac{y \eta_{x}}{1+\eta}\right\} \mathrm{d} y, \\
f_{4}(u)=- & \frac{\Phi_{y y}}{1+\eta}-\left[(1+\eta)\left(\Phi_{x}-\frac{y \eta_{x} \Phi_{y}}{1+\eta}\right)\right]_{x}+\left[\left(\Phi_{x}-\frac{y \eta_{x} \Phi_{y}}{1+\eta}\right) y \eta_{x}\right]_{y} \\
& +\frac{W(y \xi)_{y}}{1+\eta}\left(\frac{1+\eta_{x}^{2}}{\beta^{2}-W^{2}}\right)^{1 / 2},
\end{aligned}
$$

and

$$
B(u)=-y \eta_{x}+y \eta_{x} \Phi_{x}+\frac{\eta \Phi_{y}}{1+\eta}-\frac{y^{2} \eta_{x}^{2} \Phi_{y}}{1+\eta}+\frac{W y \xi}{1+\eta}\left(\frac{1+\eta_{x}^{2}}{\beta^{2}-W^{2}}\right)^{1 / 2}
$$


where

$$
W=\omega+\frac{1}{1+\eta} \int_{0}^{1} y \Phi_{y} \xi \mathrm{d} y
$$

note the helpful identity

$$
f_{4}(u)=-\left[(1+\eta) \Phi_{x}-y \eta_{x} \Phi_{y}\right]_{x}+\left[-\Phi_{y}+y \eta_{x}+B(\eta, \omega, \Phi, \xi)\right]_{y} .
$$

In keeping with our study of the Iooss-Kirchgässner line solitary waves we henceforth work in the parameter regime

$$
\alpha=\alpha_{0}+\varepsilon^{2}, \quad \beta=\beta_{0} .
$$

To identify an appropriate functional-analytic setting for these equations, let us first specialise to stationary solutions, which satisfy

$$
u_{z}=f(u)
$$

with boundary conditions

$$
\Phi_{y}=B(u) \quad \text { on } y=0,1 .
$$

Define

$$
X^{s}=H^{s+1}(\mathbb{R}) \times H^{s}(\mathbb{R}) \times H^{s+1}(\Sigma) \times H^{s}(\Sigma), \quad s \geq 0,
$$

where $\Sigma$ is the strip $\mathbb{R} \times(0,1)$, and let $M$ be an open neighbourhood of the origin in $X^{1}$ contained in the set

$$
\left\{u \in X^{1}:|W(x)|<\beta, \eta(x)>-1 \text { for all } x \in \mathbb{R}\right\}
$$

(note that $M$ is a manifold domain of $X^{0}$ ). The results presented by Bagri \& Groves [3, Proposition 2.1]) show that $B, f: M \rightarrow H^{1}(\Sigma)$ are analytic mappings. Equations (14), (15) therefore constitute an evolutionary equation in the infinite-dimensional phase space $X^{0}$ with

$$
\mathcal{D}(f)=\left\{u \in M: \Phi_{y}=B(u) \text { on } y=0,1\right\} .
$$

Furthermore, $f$ is the Hamiltonian vector field for the Hamiltonian system $\left(X^{0}, \Omega, H\right)$, where the position-independent symplectic form $\Omega$ on $X^{0}$ is given by

$$
\Omega\left(u_{1}, u_{2}\right)=\int_{\mathbb{R}}\left(\omega_{2} \eta_{1}-\eta_{2} \omega_{1}\right) \mathrm{d} x+\int_{\Sigma}\left(\xi_{2} \Phi_{1}-\Phi_{2} \xi_{1}\right) \mathrm{d} y \mathrm{~d} x
$$

and $H: M \rightarrow \mathbb{R}$ is the analytic function defined by

$$
\begin{gathered}
H(u)=\int_{\Sigma}\left\{(1+\eta) \Phi_{x}-y \eta_{x} \Phi_{y}+\frac{\xi^{2}-\Phi_{y}^{2}}{2(1+\eta)}-\frac{(1+\eta)}{2}\left(\Phi_{x}-\frac{y \eta_{x} \Phi_{y}}{1+\eta}\right)^{2}\right\} \mathrm{d} y \mathrm{~d} x \\
+\int_{\mathbb{R}}\left\{-\frac{1}{2}\left(\alpha_{0}+\varepsilon^{2}\right) \eta^{2}+\beta_{0}-\left(\beta_{0}^{2}-W^{2}\right)^{1 / 2}\left(1+\eta_{x}^{2}\right)^{1 / 2}\right\} \mathrm{d} x
\end{gathered}
$$

(see Groves, Haragus \& Sun [14, §2]). This Hamiltonian system is reversible, that is, Hamilton's equations are invariant under the transformation

$$
z \mapsto-z, \quad u \mapsto S(u)
$$

where the reverser $S: X^{s} \rightarrow X^{s}$ is defined by $S(\eta, \omega, \Phi, \xi)=(\eta,-\omega, \Phi,-\xi)$. 
Equation (14) is also invariant under the reflection $R: X^{s} \rightarrow X^{s}$ given by

$$
R(\eta(x), \omega(x), \Phi(x, y), \xi(x, y))=(\eta(-x), \omega(-x),-\Phi(-x, y),-\xi(-x, y))
$$

and one may seek solutions which are invariant under this symmetry by replacing $X^{s}$ by

$$
\begin{aligned}
X_{\mathrm{r}}^{s} & :=X^{s} \cap \operatorname{Fix} R \\
& =H_{\mathrm{e}}^{s+1}(\mathbb{R}) \times H_{\mathrm{e}}^{s}(\mathbb{R}) \times H_{\mathrm{o}}^{s+1}(\Sigma) \times H_{\mathrm{o}}^{s}(\Sigma),
\end{aligned}
$$

where

$$
\begin{aligned}
& H_{\mathrm{e}}^{s}(\mathbb{R})=\left\{w \in H^{s}(\mathbb{R}): w(x)=w(-x) \text { for all } x \in \mathbb{R}\right\} \\
& H_{\mathrm{o}}^{s}(\Sigma)=\left\{w \in H^{s}(\Sigma): w(x, y)=-w(-x, y) \text { for all }(x, y) \in \Sigma\right\}
\end{aligned}
$$

(with corresponding definitions for $H_{\mathrm{o}}^{s}(\mathbb{R})$ and $H_{\mathrm{e}}^{s}(\Sigma)$ ) and $M$ by $M_{\mathrm{r}}:=M \cap$ Fix $R$. It is also possible to extend $M_{\mathrm{r}}$ by replacing $X_{\mathrm{r}}^{1}$ in its definition with the extended function space $X_{\star}^{1}$, where

$$
X_{\star}^{s}=H_{\mathrm{e}}^{s+1}(\mathbb{R}) \times H_{\mathrm{e}}^{s}(\mathbb{R}) \times H_{\star, \mathrm{o}}^{s+1}(\Sigma) \times H_{\mathrm{o}}^{s}(\Sigma)
$$

and

$$
H_{\star, \mathrm{o}}^{s+1}(\Sigma)=\left\{w \in L_{\mathrm{loc}}^{2}(\Sigma): w_{x}, w_{y} \in H^{s}(\Sigma), w(x, y)=-w(-x, y) \text { for all }(x, y) \in \Sigma\right\}
$$

(a Banach space with norm $\|u\|_{\star, s+1}:=\left(\left\|u_{x}\right\|_{s}^{2}+\left\|u_{y}\right\|_{s}^{2}\right)^{1 / 2}$ ); note the relationship $M_{\mathrm{r}}=M_{\star} \cap X_{\mathrm{r}}^{1}$ between $M_{\mathrm{r}}$ and its extension $M_{\star}$. This feature allows one to consider solutions to (14), (15) whose $\Phi$-component is unbounded; in particular line solitary waves fall into this category.

The line solitary waves found by Iooss $\&$ Kirchgässner are equilibrium solutions of equation [14, , 215) of the form $u^{\star}=\left(\eta^{\star}, 0, \Phi^{\star}, 0\right) \in M_{\star}$, where $\eta^{\star}: \mathbb{R} \rightarrow \mathbb{R}$ and $\Phi^{\star}: \bar{\Sigma} \rightarrow \mathbb{R}$ are smooth functions whose derivatives are all $O(\varepsilon)$ (see the Appendix), and we seek solutions of the form

$$
u=u^{\star}+u^{\prime}, \quad u^{\prime} \in M^{\prime},
$$

where $M^{\prime}$ is an open neighbourhood of the origin in $X^{1}$ chosen small enough so that $u^{\star}+M^{\prime} \subseteq$ $M$; note that we can here replace $M$ with $M_{\mathrm{r}}$ or $M_{\star}$. Substituting this Ansatz into (14), (15) and again dropping the prime for notational convenience, we find that

$$
u_{z}=f\left(u^{\star}+u\right)
$$

with boundary conditions

$$
\Phi_{y}=B_{1}(\eta, \Phi)+B_{\mathrm{nl}}(\eta, \omega, \Phi, \xi) \quad \text { on } y=0,1
$$

where

$$
\begin{aligned}
B_{1}(\eta, \Phi)=\mathrm{d} B\left[\eta^{\star}, 0, \Phi^{\star}, 0\right](\eta, \omega, \Phi, \xi) & \\
=y( & \left.-\eta_{x}+\eta_{x}^{\star} \Phi_{x}+\Phi_{x}^{\star} \eta_{x}\right) \\
& \quad+\frac{\eta^{\star} \Phi_{y}}{1+\eta^{\star}}+\frac{\Phi_{y}^{\star} \eta}{\left(1+\eta^{\star}\right)^{2}}+\frac{y^{2}\left(\eta_{x}^{\star}\right)^{2} \Phi_{y}^{\star} \eta}{\left(1+\eta^{\star}\right)^{2}}-\frac{y^{2}\left(\eta_{x}^{\star}\right)^{2} \Phi_{y}}{1+\eta^{\star}}-\frac{2 y^{2} \eta_{x}^{\star} \Phi_{y}^{\star} \eta_{x}}{1+\eta^{\star}}
\end{aligned}
$$


and

$$
B_{\mathrm{nl}}(\eta, \omega, \Phi, \xi)=B\left(\eta^{\star}+\eta, \omega, \Phi^{\star}+\Phi, \xi\right)-B\left(\eta^{\star}, 0, \Phi^{\star}, 0\right)-B_{\mathrm{l}}(\eta, \Phi) .
$$

A small-amplitude periodic solution of (16), (17) corresponds to a periodically modulated solitary wave in the form of a three-dimensional perturbation of a line solitary wave.

Let us now return to $(11),(12)$. Observing that $d \in \mathcal{L}\left(X_{0}, X_{0}\right)$, one finds that these equations constitute a reversible evolutionary equation with phase space $H^{1}\left((-T, T), X_{0}\right)$; the domain of its vector field is

$$
\left\{u \in H^{2}\left((-T, T), X_{0}\right) \cap H^{1}((-T, T), M): \Phi_{y}=y \eta_{t}+B(u) \text { on } y=0,1\right\}
$$

and its reverser is given by the pointwise extension of $S: X_{0} \rightarrow X_{0}$ to $H^{1}\left((-T, T), X_{0}\right)$. Seeking solutions of the form $u=u^{\star}+u^{\prime}$, where $u^{\prime}$ has pointwise values in $M^{\prime}$, we find that

$$
u_{z}=d u_{t}+f\left(u^{\star}+u\right)
$$

with boundary conditions

$$
\Phi_{y}=y \eta_{t}+B_{\mathrm{l}}(\eta, \Phi)+B_{\mathrm{nl}}(\eta, \omega, \Phi, \xi) \quad \text { on } y=0,1,
$$

where we have again dropped the primes. We say that the solution $u^{\star}$ of (11), (12) is transversely linearly unstable if the linear equation

$$
u_{z}=d u_{t}+\mathrm{d} f\left[u^{\star}\right](u)
$$

with linear boundary conditions

$$
\Phi_{y}=y \eta_{t}+B_{1}(\eta, \Phi) \quad \text { on } y=0,1
$$

has a solution of the form $\mathrm{e}^{\sigma t} u_{\sigma}(z)$, with $u_{\sigma} \in C_{\mathrm{b}}^{1}\left(\mathbb{R}, X^{0}\right) \cap C_{\mathrm{b}}\left(\mathbb{R}, X^{1}\right)$ and $\operatorname{Re} \sigma>0$.

\subsection{Boundary conditions}

Equations (16), (17) cannot be studied using standard methods for evolutionary equations because of the nonlinear boundary conditions. This difficulty is handled using a change of variable which leads to an equivalent problem in a linear space. For $(\eta, \omega, \Phi, \xi)$ in $M, M_{\mathrm{r}}$ or $M_{\star}$ define $Q(\eta, \omega, \Phi, \xi)=(\eta, \omega, \Gamma, \xi)$, where

$$
\Gamma=\Phi+\Theta_{y}
$$

and $\Theta \in H^{3}(\Sigma)$ is the unique solution of the boundary-value problem

$$
\begin{aligned}
-\Delta \Theta+B_{1}\left(0, \Theta_{y}\right) & =B_{\mathrm{nl}}(\eta, \omega, \Phi, \xi) & & \text { in } \Sigma, \\
\Theta & =0 & & \text { on } y=0,1
\end{aligned}
$$

(the linear operator on the left-hand side of this boundary-value problem is uniformly strongly elliptic with smooth coefficients); note in particular that

$$
-\Phi_{y}+B_{1}(\eta, \Phi)+B_{\mathrm{nl}}(\eta, \omega, \Phi, \xi)=-\Gamma_{y}+B_{\mathrm{l}}(\eta, \Gamma)-\Theta_{x x}
$$

The following result is established by the method used by Groves, Haragus \& Sun [14, Lemma 2.1]. 


\section{Lemma 2.1}

(i) The mapping $Q$ is a near-identity, analytic diffeomorphism from a neighbourhood $M$ of the origin in $X^{1}$ onto a neighbourhood $\tilde{M}$ of the origin in $X^{1}$.

(ii) For each $u \in M$ the operator $\mathrm{d} Q[u]$ also defines an isomorphism $\widehat{\mathrm{d} Q}[u]: X^{0} \rightarrow X^{0}$, and the operators $\widehat{\mathrm{d} Q}[u], \widehat{\mathrm{d} Q}[u]^{-1} \in \mathcal{L}\left(X^{0}, X^{0}\right)$ depend analytically on $u \in M$.

(iii) Statements (i) and (ii) also hold when $M, \tilde{M}$ are replaced by $M_{\mathrm{r}}, \tilde{M}_{\mathrm{r}}$ or $M_{\star}, \tilde{M}_{\star}$, where $M_{\mathrm{r}}=M \cap \operatorname{Fix} R=M_{\star} \cap X_{\mathrm{r}}^{1}$ and $\tilde{M}_{\mathrm{r}}=\tilde{M} \cap \operatorname{Fix} R=\tilde{M}_{\star} \cap X_{\mathrm{r}}^{1}$.

The above change of variable transforms $(16)$ into the equation

$$
v_{z}=L v+N(v)
$$

with linear boundary conditions

$$
\Gamma_{y}=B_{1}(\eta, \Gamma) \quad \text { on } y=0,1
$$

for the variable $v=Q(u)$, in which $L:=\mathrm{d} \tilde{f}[0]=\mathrm{d} f\left[u^{\star}\right]$ and $N:=\tilde{f}-L$ are the linear and nonlinear parts of the transformed vector field

$$
\tilde{f}(v):=\widehat{\mathrm{d} Q}\left[Q^{-1}(v)\right]\left(f\left(u^{\star}+Q^{-1}(v)\right)\right) .
$$

Here

$$
\mathcal{D}(L)=Y_{1}:=\left\{(\eta, \omega, \Gamma, \xi) \in X^{1}: \Gamma_{y}=B_{1}(\eta, \Gamma) \text { on } y=0,1\right\}
$$

and $\mathcal{D}(N)$ is the neighbourhood $U:=\tilde{M} \cap Y^{1}$ of the origin in $Y^{1}$, and we may replace $\tilde{M}, Y^{1}, U$ by $\tilde{M}_{\mathrm{r}}, Y_{\mathrm{r}}^{1}, U_{\mathrm{r}}$ or $\tilde{M}_{\star}, Y_{\star}^{1}, U_{\star}$ in the usual fashion. The linear operator $L$ is given by the explicit formula

$$
L\left(\begin{array}{l}
\eta \\
\omega \\
\Gamma \\
\xi
\end{array}\right)=\left(\begin{array}{c}
\frac{\omega}{\beta_{0}}+h_{1}(\omega, \xi) \\
\left(\alpha_{0}+\varepsilon^{2}\right) \eta-\left.\Gamma_{x}\right|_{y=1}-\beta_{0} \eta_{x x}+h_{2}(\eta, \Gamma) \\
\xi+H_{1}(\omega, \xi) \\
-\Gamma_{x x}-\Gamma_{y y}+H_{2}(\eta, \Gamma),
\end{array}\right),
$$

where

$$
\begin{aligned}
& h_{1}(\omega, \xi)=\frac{\left(1+\left(\eta_{x}^{\star}\right)^{2}\right)^{1 / 2}}{\beta_{0}}\left(\omega+\frac{1}{1+\eta^{\star}} \int_{0}^{1} y \Phi_{y}^{\star} \xi \mathrm{d} y\right)-\frac{\omega}{\beta_{0}}, \\
& h_{2}(\eta, \Gamma)=\beta_{0} \eta_{x x}-\beta_{0}\left[\frac{\eta_{x}}{\left(1+\left(\eta_{x}^{\star}\right)^{2}\right)^{3 / 2}}\right]_{x} \\
& +\int_{0}^{1}\left\{\Phi_{x}^{\star} \Gamma_{x}-\frac{\Phi_{y}^{\star} \Gamma_{y}}{\left(1+\eta^{\star}\right)^{2}}+\frac{\left(\Phi_{y}^{\star}\right)^{2} \eta}{\left(1+\eta^{\star}\right)^{3}}-\frac{y^{2}\left(\eta_{x}^{\star}\right)^{2} \Phi_{y}^{\star} \Gamma_{y}}{\left(1+\eta^{\star}\right)^{2}}-\frac{y^{2} \eta_{x}^{\star}\left(\Phi_{y}^{\star}\right)^{2} \eta_{x}}{\left(1+\eta^{\star}\right)^{2}}+\frac{y^{2} \eta_{x}^{\star}\left(\Phi_{y}^{\star}\right)^{2} \eta}{\left(1+\eta^{\star}\right)^{3}}\right. \\
& \left.\quad+\left[y \Phi_{y}^{\star} \Gamma_{x}+y \Phi_{x}^{\star} \Gamma_{y}-\frac{2 y^{2} \eta_{x}^{\star} \Phi_{y}^{\star} \Gamma_{y}}{1+\eta^{\star}}-\frac{y^{2}\left(\Phi_{y}^{\star}\right)^{2} \eta_{x}}{1+\eta^{\star}}+\frac{y^{2}\left(\Phi_{y}^{\star}\right)^{2} \eta_{x}^{\star} \eta}{\left(1+\eta^{\star}\right)^{2}}\right]_{x}\right\} \mathrm{d} y, \\
& H_{1}(\omega, \xi)=-\frac{1}{1+\eta^{\star} \xi}+\frac{\left(1+\left(\eta_{x}^{\star}\right)^{2}\right)^{1 / 2}}{\beta_{0}\left(1+\eta^{\star}\right)}\left(\omega+\frac{1}{1+\eta^{\star}} \int_{0}^{1} y \Phi_{y}^{\star} \xi \mathrm{d} y\right) y \Phi_{y}^{\star}, \\
& H_{2}(\eta, \Gamma)=\left(F_{1}(\eta, \Gamma)\right)_{x}+\left(F_{3}(\eta, \Gamma)\right)_{y}
\end{aligned}
$$


and

$$
\begin{aligned}
& F_{1}(\eta, \Gamma)=-\eta^{\star} \Gamma_{x}-\Phi_{x}^{\star} \eta+y \Phi_{y}^{\star} \eta_{x}+y \eta_{x}^{\star} \Gamma_{y}, \\
& F_{3}(\eta, \Gamma)=y \eta_{x}+B_{1}(\eta, \Gamma) .
\end{aligned}
$$

Furthermore, the change of variable preserves the reversibility of the evolutionary equation.

A similar issue arises when seeking solutions of (18), (19) which depend analytically upon $t$, which we regard as a parameter. In this setting the equations constitute an evolutionary system with phase space $\tilde{X}^{0}$, the domain of whose vector field is the subset of functions in $\tilde{X}^{1}$ which satisfy (19); here the tilde denotes the analytic dependence upon $t$. Standard methods cannot be applied because of the dependence of the boundary conditions upon the parameter, and this problem is also resolved using a change of variables. Define

$$
\Gamma=\Phi+\Theta_{y t},
$$

where $\Theta$ is the unique solution of the boundary-value problem

$$
\begin{aligned}
-\Delta \Theta+B_{1}\left(0, \Theta_{y}\right) & =y \eta & & \text { in } \Sigma, \\
\Theta & =0 & & \text { on } y=0,1 .
\end{aligned}
$$

The change of variable $u=(\eta, \omega, \Phi, \xi) \mapsto(\eta, \omega, \Gamma, \xi)=v$ is a linear isomorphism $\tilde{X}^{0} \rightarrow \tilde{X}^{0}$ and $\tilde{X}^{1} \rightarrow \tilde{X}^{1}$. Note in particular that

$$
-\Phi_{y}+y \eta_{t}+B_{1}(\eta, \Phi)=-\Gamma_{y}+B_{1}(\eta, \Gamma)-\Theta_{x x t},
$$

so that this change of variable transforms $(18),(19)$ into

$$
v_{z}=d_{1} v_{t}+d_{2} v_{t t}+L v
$$

with boundary conditions

$$
\Gamma_{y}=B_{1}(\eta, \Gamma) \quad \text { on } y=0,1,
$$

where $d_{1}, d_{2} \in \mathcal{L}\left(\tilde{X}^{0}, \tilde{X}^{0}\right)$ are given by

$$
d_{1}\left(\begin{array}{c}
\eta \\
\omega \\
\Gamma \\
\xi
\end{array}\right)=\left(\begin{array}{c}
0 \\
\left.\Gamma\right|_{y=1}+\left.\Theta_{x y}\right|_{y=1}-h_{2}\left(0, \Theta_{y}\right) \\
\tilde{\Theta}_{y} \\
-\eta-\left(F_{1}\left(0, \Theta_{y}\right)\right)_{x}
\end{array}\right), \quad d_{2}\left(\begin{array}{l}
\eta \\
\omega \\
\Gamma \\
\xi
\end{array}\right)=\left(\begin{array}{c}
0 \\
-\left.\Theta_{y}\right|_{y=1} \\
0 \\
0
\end{array}\right)
$$

and $\tilde{\Theta}$ is the unique solution of the boundary-value problem

$$
\begin{aligned}
-\Delta \tilde{\Theta}+B_{1}\left(0, \tilde{\Theta}_{y}\right) & =y\left(\frac{\omega}{\beta_{0}}+h_{1}(\omega, \xi)\right) & & \text { in } \Sigma, \\
\tilde{\Theta} & =0 & & \text { on } y=0,1 .
\end{aligned}
$$




\subsection{Existence results}

We construct periodically modulated solitary waves by applying the Lyapunov-Iooss theorem (Theorem 1.1) to 21), 22), taking $\mathcal{X}=X_{\mathrm{r}}^{0}, \mathcal{Y}=Y_{\star}^{1}, \mathcal{Z}=Y_{\mathrm{r}}^{1}$ and $\mathcal{U}=U_{\star}$; similarly, we demonstrate the transverse linear instability of the Iooss-Kirchgässner solitary waves by applying Theorem 1.3 to 23 , 24, taking $\mathcal{X}=X^{0}$ and $\mathcal{Z}=Y^{1}$, to construct a solution of the form $\mathrm{e}^{\sigma t} v_{\sigma}(z)$, where $v_{\sigma} \in C^{1}\left(\mathbb{R}, X^{0}\right) \cap C\left(\mathbb{R}, Y^{1}\right)$ is periodic. (In both cases we of course take $\tau=z$ and $S(\eta, \omega, \Gamma, \xi)=(\eta,-\omega, \Gamma,-\xi)$.) The spectral hypotheses are verified by studying the resolvent equations

$$
(L-\mathrm{i} \lambda I) u=u^{\dagger}, \quad \lambda \in \mathbb{R},
$$

for $L$, that is

$$
\begin{aligned}
\frac{\omega}{\beta_{0}}+h_{1}(\omega, \xi) & =\mathrm{i} \lambda \eta+\eta^{\dagger}, \\
\left(\alpha_{0}+\varepsilon^{2}\right) \eta-\left.\Gamma_{x}\right|_{y=1}-\beta_{0} \eta_{x x}+h_{2}(\eta, \Gamma) & =\mathrm{i} \lambda \omega+\omega^{\dagger}, \\
\xi+H_{1}(\omega, \xi) & =\mathrm{i} \lambda \Gamma+\Gamma^{\dagger}, \\
-\Gamma_{x x}-\Gamma_{y y}+\left(F_{1}(\eta, \Gamma)\right)_{x}+\left(F_{3}(\eta, \Gamma)\right)_{y} & =\mathrm{i} \lambda \xi+\xi^{\dagger}
\end{aligned}
$$

with

$$
\begin{array}{ll}
\Gamma_{y}=0 & \text { on } y=0 \\
\Gamma_{y}=-\eta_{x}+F_{3}(\eta, \Gamma) & \text { on } y=1
\end{array}
$$

since $L$ is real and anticommutes with the reverser $S$ it suffices to examine non-negative values of $\lambda$, real values of $\eta, \Gamma, \omega^{\dagger}, \xi^{\dagger}$ and imaginary values of $\omega, \xi, \eta^{\dagger}, \Gamma^{\dagger}$.

The arguments given in Section 1 suggest that the support of the Fourier transform $\hat{\eta}=\mathcal{F}[\eta]$ of $\eta$ is concentrated near wavenumbers $\mu= \pm \mu_{0}$; we therefore decompose it into the sum of a function $\hat{\eta}_{1}$ with spectrum near $\mu= \pm \mu_{0}$ and a function $\hat{\eta}_{2}$ whose support is bounded away from these points. To this end choose $\delta \in\left(0, \mu_{0} / 3\right)$, let $\chi_{0}$ be the characteristic function of the interval $[-\delta, \delta]$ and define

$$
\eta_{1}=\chi(D) \eta:=\mathcal{F}^{-1}[\chi \hat{\eta}], \quad \eta_{2}=(1-\chi(D)) \eta:=\mathcal{F}^{-1}[(1-\chi) \hat{\eta}],
$$

where $\chi(\mu)=\chi_{0}\left(\mu-\mu_{0}\right)+\chi_{0}\left(\mu+\mu_{0}\right)$. In Section 3 we reduce (25)-(30) to a linear equation of Davey-Stewartson type by determining $\eta_{2}, \omega, \Gamma$ and $\xi$ as functions of $\eta_{1}$ and writing

$$
\eta_{1}(x)=\frac{1}{2} \varepsilon \zeta(\varepsilon x) \mathrm{e}^{\mathrm{i} \mu_{0} x}+\frac{1}{2} \varepsilon \overline{\zeta(\varepsilon x)} \mathrm{e}^{-\mathrm{i} \mu_{0} x}
$$

for some $\zeta \in \chi_{0}(\varepsilon D) L^{2}(\mathbb{R})$, so that $\eta_{1} \in \chi(D) L^{2}(\mathbb{R})$. The results of these calculations are summarised in the following theorem.

\section{Theorem 2.2}

(i) There exists a constant $k_{\max }>0$ such that equations (25)-(30) have a unique solution $u \in Y^{1}$ for each $\lambda>\varepsilon k_{\max }$ and each $u^{\dagger} \in X^{0}$. 
(ii) Suppose that $\lambda=\varepsilon k$, where $0<k \leq k_{\max }$. There exist $\zeta_{\varepsilon, k}^{\dagger} \in \mathcal{L}\left(X^{0}, \chi_{0}(\varepsilon D) L^{2}(\mathbb{R})\right)$ and an injection $\check{u}_{\varepsilon, k} \in \mathcal{L}\left(\chi_{0}(\varepsilon D) L^{2}(\mathbb{R}) \times X^{0}, Y^{1}\right)$ such that $u \in Y^{1}$ solves the resolvent equations

$$
(L-\mathrm{i} \lambda I) u=u^{\dagger}
$$

if and only if $u=\breve{u}_{\varepsilon, k}\left(\zeta, u^{\dagger}\right)$ for some solution of the reduced equation

$$
\mathcal{B}_{\varepsilon, k}(\zeta, \psi)=\left(\zeta_{\varepsilon, k}^{\dagger}\left(u^{\dagger}\right), 0\right)
$$

The operator $\mathcal{B}_{\varepsilon, k}: \mathcal{D}_{\mathcal{B}} \subseteq W \rightarrow W$ is given by

$\mathcal{B}_{\varepsilon, k}(\zeta, \psi)=$

$$
\left(\begin{array}{c}
A_{2}^{-1} \zeta-A_{2}^{-1} A_{1} \zeta_{x x}+k^{2} \zeta-A_{2}^{-1}\left(A_{3}+A_{5}\right) \zeta^{\star 2} \zeta-A_{2}^{-1} A_{3} \zeta^{\star 2} \bar{\zeta}+4 A_{2}^{-1} A_{4} \zeta^{\star} \psi_{x}-\mathcal{R}_{\varepsilon, k}(\zeta) \\
-\left(1-\alpha_{0}^{-1}\right) \psi_{x x}+k^{2} \psi-2 A_{4} \operatorname{Re}\left(\zeta^{\star} \zeta\right)_{x}
\end{array}\right),
$$

where $W=L^{2}(\mathbb{R}) \times L^{2}(\mathbb{R}), \mathcal{D}_{\mathcal{B}}=H^{2}(\mathbb{R}) \times H^{2}(\mathbb{R})$ and $\mathcal{R}_{\varepsilon, k} \in \mathcal{L}\left(H^{2}(\mathbb{R}), L^{2}(\mathbb{R})\right)$ satisfies the estimate

$$
\left\|\mathcal{R}_{\varepsilon, k}(\zeta)\right\|_{0} \leq c \varepsilon\|\zeta\|_{1}
$$

each solution $(\zeta, \psi)$ of the reduced equation satisfies $\zeta \in \chi_{0}(\varepsilon D) H^{2}(\mathbb{R})$. In particular, $L-\mathrm{i} \varepsilon k I$ is (semi-)Fredholm if $\mathcal{B}_{\varepsilon, k}$ is (semi-)Fredholm, and the kernels of $L-\mathrm{i} \varepsilon k I$ and $\mathcal{B}_{\varepsilon, k}$ have the same dimension.

Furthermore, the reduction preserves the invariance of the resolvent equations under the reflection $R$; its action on $W$ is given by $\tilde{R}:(\zeta(x), \psi(x)) \mapsto(\overline{\zeta(-x)},-\psi(-x))$.

(iii) Define $Z_{\mathrm{r}}^{0}=H_{\mathrm{e}}^{1}(\mathbb{R}) \times L_{\mathrm{e}}^{2}(\mathbb{R}) \times H_{\mathrm{o}}^{1}(\Sigma) \times H_{\mathrm{e}}^{1}(\Sigma) \times \stackrel{\circ}{H}_{\mathrm{o}}^{1}(\Sigma)$, where

$$
\stackrel{\circ}{H}_{\mathrm{o}}^{1}(\Sigma)=\left\{u \in H_{\mathrm{o}}^{1}(\Sigma):\left.u\right|_{y=0}=\left.u\right|_{y=1}=0\right\},
$$

and

$$
H_{\mathrm{c}}^{s}(\mathbb{R})=\left\{u \in H^{s}(\mathbb{R}): u(-x)=\overline{u(x)} \text { for all } x \in \mathbb{R}\right\}, \quad s \geq 0 .
$$

There exist $\zeta_{\varepsilon, 0}^{\dagger} \in \mathcal{L}\left(Z_{\mathrm{r}}^{0}, \chi_{0}(\varepsilon D) L_{\mathrm{c}}^{2}(\mathbb{R})\right)$ and an injection $\check{u}_{\varepsilon, 0} \in \mathcal{L}\left(\chi_{0}(\varepsilon D) L_{\mathrm{c}}^{2}(\mathbb{R}) \times Z_{\mathrm{r}}^{0}, Y_{\star}^{1}\right)$ such that $u \in Y_{\star}^{1}$ solves

$$
L u=u^{\dagger}
$$

where $\xi^{\dagger}=-\left(\xi_{1}^{\dagger}\right)_{x}-\left(\xi_{3}^{\dagger}\right)_{y}$, if and only if $u=\check{u}_{\varepsilon, 0}\left(\zeta, \eta^{\dagger}, \omega^{\dagger}, \Gamma^{\dagger}, \xi_{1}^{\dagger}, \xi_{3}^{\dagger}\right)$ for some solution of the reduced equation

$$
\mathcal{C}_{\varepsilon} \zeta=\zeta_{\varepsilon, 0}^{\dagger}\left(\eta^{\dagger}, \omega^{\dagger}, \Gamma^{\dagger}, \xi_{1}^{\dagger}, \xi_{3}^{\dagger}\right)
$$

The operator $\mathcal{C}_{\varepsilon}: H_{\mathrm{c}}^{2}(\mathbb{R}) \subseteq L_{\mathrm{c}}^{2}(\mathbb{R}) \rightarrow L_{\mathrm{c}}^{2}(\mathbb{R})$ is given by

$$
\mathcal{C}_{\varepsilon} \zeta=A_{2}^{-1} \zeta-A_{2}^{-1} A_{1} \zeta_{x x}-2 A_{2}^{-1} A_{5} \zeta^{\star 2} \zeta-A_{2}^{-1} A_{5} \zeta^{\star 2} \bar{\zeta}-\mathcal{R}_{\varepsilon, 0}(\zeta)
$$

and $\mathcal{R}_{\varepsilon, 0} \in \mathcal{L}\left(H_{\mathrm{c}}^{2}(\mathbb{R}), L_{\mathrm{c}}^{2}(\mathbb{R})\right)$ satisfies the estimate

$$
\left\|\mathcal{R}_{\varepsilon, 0}(\zeta)\right\|_{0} \leq c \varepsilon\|\zeta\|_{1}
$$

each solution of the reduced equation lies in $\chi_{0}(\varepsilon D) H_{\mathrm{c}}^{2}(\mathbb{R})$. 
Part (i) of Theorem 2.2 implies that $i \lambda \in \rho(L)$ for $\lambda>\varepsilon k_{\max }$, while parts (ii) and (iii) show in particular that $\mathrm{i} \varepsilon k \in \rho(L)$ if $\mathcal{B}_{\varepsilon, k}$ (for $k>0$ ) or $\mathcal{C}_{\varepsilon}$ (for $k=0$ ) is invertible. We study the invertibility of these operators in Section 4, establishing the following results, whose corollaries relate them to the operator $L$.

Lemma 2.3 For each sufficiently small $k_{\min }>0$ there exists a unique number $k_{\varepsilon} \in\left[k_{\min }, k_{\max }\right]$ with the following properties.

(i) The operator $\mathcal{B}_{\varepsilon, k}: \mathcal{D}_{\mathcal{B}} \subseteq W \rightarrow W$ is an isomorphism for each $k \in\left[k_{\min }, k_{\max }\right] \backslash\left\{k_{\varepsilon}\right\}$.

(ii) The operator $\mathcal{B}_{\varepsilon, k_{\varepsilon}}: \mathcal{D}_{\mathcal{B}} \subseteq W \rightarrow W$ is Fredholm with index 0 and has a onedimensional kernel which lies in Fix $\tilde{R}$.

Corollary 2.4 The imaginary number i $\varepsilon$ k belongs to $\rho(L)$ for each $k \in\left[k_{\min }, \infty\right) \backslash\left\{k_{\varepsilon}\right\}$, while $\mathrm{i} \varepsilon k_{\varepsilon}$ is a simple eigenvalue of $L$ whose eigenspace lies in Fix $R$.

Proof. It remains only to show that the eigenvalue $\mathrm{i} \varepsilon k_{\varepsilon}$ is algebraically simple. Observe that $\Omega\left(L u_{1}, u_{2}\right)=-\Omega\left(u_{1}, L u_{2}\right)$ and in particular that $\Omega\left(\left(L-\mathrm{i} \varepsilon k_{\varepsilon}\right) u_{1}, u_{2}\right)=-\Omega\left(u_{1},\left(L+\mathrm{i} \varepsilon k_{\varepsilon}\right) u_{2}\right)$ for $u_{1}, u_{2} \in \mathcal{D}(L)$. It follows that $\Omega\left(f, \bar{u}_{\varepsilon}\right)=0$ is a necessary condition for $f \in X_{\mathrm{r}}^{0}$ to lie in the range of $L-\mathrm{i} \varepsilon k_{\varepsilon} I$, where $u_{\varepsilon}$ is an eigenvector corresponding to the eigenvalue $k_{\varepsilon}$. Using the formulae

$$
\begin{aligned}
\omega & =\frac{\mathrm{i} \varepsilon k_{\varepsilon} \beta_{0} \eta_{\varepsilon}}{\left(1+\left(\eta_{x}^{\star}\right)^{2}\right)^{1 / 2}}-\mathrm{i} \varepsilon k_{\varepsilon} \int_{0}^{1} y \Phi_{y}^{\star} \Gamma_{\varepsilon} \mathrm{d} y+\frac{\mathrm{i} \varepsilon k_{\varepsilon} \eta_{\varepsilon}}{1+\eta^{\star}} \int_{0}^{1} y \Phi_{y}^{\star} \mathrm{d} y \\
\xi & =\left(1+\eta^{\star}\right) \mathrm{i} \varepsilon k_{\varepsilon} \Gamma_{\varepsilon}-\mathrm{i} \varepsilon k_{\varepsilon} \eta_{\varepsilon} y \Phi_{y}^{\star}
\end{aligned}
$$

(see equations (31), 32), we find that

$$
\Omega\left(u_{\varepsilon}, \bar{u}_{\varepsilon}\right)=-2 \mathrm{i} \varepsilon k_{\varepsilon} \beta_{0} \int_{\mathbb{R}}\left|\eta_{\varepsilon}\right|^{2} \mathrm{~d} x-2 \mathrm{i} \varepsilon k_{\varepsilon} \int_{\Sigma}\left|\Gamma_{\varepsilon}\right|^{2} \mathrm{~d} y \mathrm{~d} x+O\left(\varepsilon^{2}\left(\left\|\eta_{\varepsilon}\right\|_{0}^{2}+\left\|\Gamma_{\varepsilon}\right\|_{0}^{2}\right)\right) \neq 0,
$$

so that $u_{\varepsilon}$ does not lie in the range of $L-\mathrm{i} \varepsilon k_{\varepsilon} I$.

\section{Lemma 2.5}

(i) The operator $\mathcal{C}_{\varepsilon}: H_{\mathrm{c}}^{2}(\mathbb{R}) \subseteq L_{\mathrm{c}}^{2}(\mathbb{R}) \rightarrow L_{\mathrm{c}}^{2}(\mathbb{R})$ is an isomorphism.

(ii) There exists $k_{\min }>0$ such that $\left.\mathcal{B}_{\varepsilon, k}\right|_{\mathrm{Fix} \tilde{R}}$ is semi-Fredholm and injective for $k \in$ $\left(0, k_{\min }\right)$.

Corollary 2.6 The operator $L-\mathrm{i} \varepsilon k I: Y_{\mathrm{r}}^{1} \rightarrow X_{\mathrm{r}}^{0}$ is an isomorphism for each $k \in\left(0, k_{\min }\right)$.

Proof. Because $k \mapsto \mathcal{B}_{\varepsilon, k}$ is a continuous mapping $\left(0, k_{\text {max }}\right) \mapsto \mathcal{L}\left(\mathcal{D}_{\mathcal{B}} \cap\right.$ Fix $\tilde{R}$, Fix $\tilde{R}$ ) (see Section 4.3 and the Fredholm index of $\left.\mathcal{B}_{\varepsilon, k}\right|_{\text {Fix } \tilde{R}}$ is 0 for $k \in\left[k_{\min }, k_{\max }\right]$, the same is true for $k \in\left(0, k_{\text {min }}\right)$. Using Lemma 2.5 , we find that $\left.\mathcal{B}_{\varepsilon, k}\right|_{\text {Fix } \tilde{R}}$ is invertible for $k \in\left(0, k_{\text {min }}\right)$, and the stated result follows from Theorem 2.2(ii).

The resolvent decay estimate is a consequence of the next lemma, whose proof is given by Groves, Haragus \& Sun [14, Lemma 3.25]. 
Lemma 2.7 There exists a constant $\lambda^{\star}>0$ such that the solution $u \in Y^{1}$ of equations (25)-(30) satisfies the estimate

$$
\|u\|_{X^{1}}^{2}+\lambda^{2}\|u\|_{X^{0}}^{2} \leq c\left\|u^{\dagger}\right\|_{X^{0}}^{2}
$$

for each $\lambda>\lambda^{\star}$ and each $u^{\dagger} \in X_{\mathrm{r}}^{0}$.

It remains to establish the Iooss condition at the origin. For this purpose we first record the following consequence of Theorem 2.2(iii) and Lemma 2.5 (i).

Proposition 2.8 For each $\eta^{\dagger} \in H_{\mathrm{e}}^{1}(\mathbb{R}), \omega^{\dagger} \in L_{\mathrm{e}}^{2}(\mathbb{R}), \Gamma^{\dagger} \in H_{\mathrm{o}}^{s}(\Sigma)$ and

$$
\xi^{\dagger}=-\left(\xi_{1}^{\dagger}\right)_{x}-\left(\xi_{3}^{\dagger}\right)_{y}
$$

where $\xi_{1}^{\dagger} \in H_{\mathrm{e}}^{1}(\Sigma)$ and $\xi_{3}^{\dagger} \in \stackrel{\circ}{H}_{\mathrm{o}}^{1}(\Sigma)$, the equation

$$
L u=u^{\dagger}
$$

has a unique solution $u \in Y_{\star}^{1}$ which satisfies the estimate

$$
\|u\|_{Y_{\star}^{1}} \leq c\left(\left\|\eta^{\dagger}\right\|_{1}+\left\|\omega^{\dagger}\right\|_{0}+\left\|\Gamma^{\dagger}\right\|_{1}+\left\|\xi_{1}^{\dagger}\right\|_{1}+\left\|\xi_{3}^{\dagger}\right\|_{1}\right) .
$$

Lemma 2.9 For each $v^{\dagger} \in U_{\star}$ the equation

$$
L v=-N\left(v^{\dagger}\right)
$$

has a unique solution $v \in Y_{\star}^{1}$ which satisfies the estimate

$$
\|v\|_{Y_{\star}^{1}} \leq c\left\|v^{\dagger}\right\|_{Y_{\star}^{1}} .
$$

Proof. Choose $v^{\dagger} \in U_{\star}$ and write $u^{\dagger}=Q^{-1}\left(v^{\dagger}\right)$.

Recall that

$$
g(v)=\widehat{\mathrm{d} Q}[u]\left(f\left(u^{\star}+u\right)\right), \quad u=Q^{-1}(v)
$$

and that $Q, \widehat{\mathrm{d} Q}[u]$ do not alter the fourth component of their arguments. The fourth component of $g\left(v^{\dagger}\right)$ is therefore given by

$$
\begin{aligned}
g_{4}\left(v^{\dagger}\right)= & f_{4}\left(u^{\star}+u^{\dagger}\right) \\
=- & {\left[\left(1+\eta^{\star}+\eta^{\dagger}\right)\left(\Phi_{x}^{\star}+\Gamma_{x}^{\dagger}\right)-y\left(\eta_{x}^{\star}+\eta_{x}^{\dagger}\right)\left(\Phi_{y}^{\star}+\Gamma_{y}^{\dagger}\right)\right]_{x} } \\
& \quad+\left[-\Phi_{y}^{\star}-\Gamma_{y}^{\dagger}+B\left(\eta^{\star}+\eta^{\dagger}, \omega^{\dagger}, \Phi^{\star}+\Gamma^{\dagger}, \xi^{\dagger}\right)+y\left(\eta_{x}^{\star}+\eta_{x}^{\dagger}\right)\right]_{y} \\
=- & {\left[\left(1+\eta^{\star}+\eta^{\dagger}\right) \Gamma_{x}^{\dagger}+\Phi_{x}^{\star} \eta^{\dagger}-y\left(\eta_{x}^{\star}+\eta_{x}^{\dagger}\right) \Gamma_{y}^{\dagger}-y \Phi_{y}^{\star} \eta_{x}^{\dagger}\right]_{x} } \\
& \quad+\left[-\Gamma_{y}^{\dagger}+B_{1}\left(\eta^{\dagger}, \Gamma^{\dagger}\right)+B_{\mathrm{nl}}\left(\eta^{\dagger}, \omega^{\dagger}, \Gamma^{\dagger}, \xi^{\dagger}\right)+y \eta_{x}^{\dagger}\right]_{y} \\
=-[(1+ & \left.\left.\eta^{\star}+\eta^{\dagger}\right) \Gamma_{x}^{\dagger}+\Phi_{x}^{\star} \eta^{\dagger}-y\left(\eta_{x}^{\star}+\eta_{x}^{\dagger}\right) \Gamma_{y}^{\dagger}-y \Phi_{y}^{\star} \eta_{x}^{\dagger}\right]_{x} \\
\quad & \quad\left[-\Gamma_{y}^{\dagger}-\Theta_{x x}^{\dagger}+B_{1}\left(\eta^{\dagger}, \Gamma^{\dagger}\right)+y \eta_{x}^{\dagger}\right]_{y} \\
=-\left[\Gamma_{x}^{\dagger}+\right. & \left.\left(\eta^{\star}+\eta^{\dagger}\right) \Gamma_{x}^{\dagger}+\Phi_{x}^{\star} \eta^{\dagger}-y\left(\eta_{x}^{\star}+\eta_{x}^{\dagger}\right) \Gamma_{y}^{\dagger}-y \Phi_{y}^{\star} \eta_{x}^{\dagger}\right]_{x} \\
& +\left[-\Gamma_{y}^{\dagger}+B_{1}\left(\eta^{\dagger}, \Gamma^{\dagger}\right)+y \eta_{x}^{\dagger}\right]_{y}
\end{aligned}
$$


in which the first line follows from $\sqrt{13}$, the second from the fact that $f_{4}\left(u^{\star}\right)=0$ and the definitions of $B_{1}, B_{\mathrm{nl}}$, the third from 20 and the fourth from the identity $\Gamma_{x x}^{\dagger}=\Phi_{x x}^{\dagger}+\Theta_{x x y}^{\dagger}$. According to the definition $N(v)=g(v)-L v$ the fourth component of $N\left(v^{\dagger}\right)$ is

$$
\begin{aligned}
N_{4}\left(v^{\dagger}\right)= & f_{4}\left(u^{\star}+u^{\dagger}\right)-L_{4} v^{\dagger} \\
= & -\left[F_{1}\left(\eta^{\dagger}, \Gamma^{\dagger}\right)+\left(\eta^{\star}+\eta^{\dagger}\right) \Gamma_{x}^{\dagger}+\Phi_{x}^{\star} \eta^{\dagger}-y\left(\eta_{x}^{\star}+\eta_{x}^{\dagger}\right) \Gamma_{y}^{\dagger}-y \Phi_{y}^{\star} \eta_{x}^{\dagger}\right]_{x} \\
& \quad+\left[-F_{3}\left(\eta^{\dagger}, \Gamma^{\dagger}\right)+B_{1}\left(\eta^{\dagger}, \Gamma^{\dagger}\right)+y \eta_{x}^{\dagger}\right]_{y} \\
= & -\left[F_{1}\left(\eta^{\dagger}, \Gamma^{\dagger}\right)+\left(\eta^{\star}+\eta^{\dagger}\right) \Gamma_{x}^{\dagger}+\Phi_{x}^{\star} \eta^{\dagger}-y\left(\eta_{x}^{\star}+\eta_{x}^{\dagger}\right) \Gamma_{y}^{\dagger}-y \Phi_{y}^{\star} \eta_{x}^{\dagger}\right]_{x} .
\end{aligned}
$$

Applying Proposition 2.8 with

$$
\xi_{1}^{\dagger}=-\left(F_{1}\left(\eta^{\dagger}, \Gamma^{\dagger}\right)+\left(\eta^{\star}+\eta^{\dagger}\right) \Gamma_{x}^{\dagger}+\Phi_{x}^{\star} \eta^{\dagger}-y\left(\eta_{x}^{\star}+\eta_{x}^{\dagger}\right) \Gamma_{y}^{\dagger}-y \Phi_{y}^{\star} \eta_{x}^{\dagger}\right), \quad \xi_{3}^{\dagger}=0,
$$

one finds that the equation

$$
L v=-N\left(v^{\dagger}\right)
$$

has a unique solution $v \in Y_{\star}^{1}$ which satisfies

$$
\|v\|_{Y_{\star}^{1}} \leq c\left(\left\|N_{1}\left(v^{\dagger}\right)\right\|_{1}+\left\|N_{2}\left(v^{\dagger}\right)\right\|_{0}+\left\|N_{3}\left(v^{\dagger}\right)\right\|_{1}+\left\|\xi_{1}^{\dagger}\right\|_{1}\right) .
$$

The assertion follows by combining this estimate with

$$
\left\|\xi_{1}^{\dagger}\right\|_{1} \leq c\left(\left\|\eta^{\dagger}\right\|_{2}+\left\|\nabla \Gamma^{\dagger}\right\|_{1}+\left\|\nabla \Gamma^{\dagger}\right\|_{1}\right) \leq c\left\|v^{\dagger}\right\|_{Y_{\star}^{1}}
$$

and

$$
\left\|N\left(v^{\dagger}\right)\right\|_{X^{0}} \leq c\left\|v^{\dagger}\right\|_{Y_{\star}^{1}}
$$

\section{Derivation of the reduced equation}

\subsection{Reduction to a single pseudodifferential equation}

We begin by eliminating $\omega, \Gamma$ and $\xi$ from $(25)-(30)$ and deriving a single, equivalent equation for $\eta$. Equations (25) and (27) clearly yield the explicit formulae

$$
\begin{aligned}
\omega & =\frac{\beta_{0}}{\left(1+\left(\eta_{x}^{\star}\right)^{2}\right)^{1 / 2}}\left(\eta^{\dagger}+\mathrm{i} \lambda \eta\right)-\frac{1}{1+\eta^{\star}} \int_{0}^{1} y \Phi_{y}^{\star} \xi \mathrm{d} y, \\
\xi & =\left(1+\eta^{\star}\right)\left(\Gamma^{\dagger}+\mathrm{i} \lambda \Gamma\right)-y \Phi_{y}^{\star}\left(\eta^{\dagger}+\mathrm{i} \lambda \eta\right) .
\end{aligned}
$$

for $\omega$ and $\xi$, and substituting these formulae into 28$]-(30)$, we obtain the boundary-value problem

$$
\begin{aligned}
-\hat{\Gamma}_{y y}+q^{2} \hat{\Gamma} & =\hat{F}^{\dagger}\left(u^{\dagger}\right)-\mathrm{i} \mu \hat{F}_{1}(\eta, \Gamma)-\mathrm{i} \lambda \hat{F}_{2}(\eta, \Gamma)-\left(\hat{F}_{3}(\eta, \Gamma)\right)_{y}, & & 0<y<1, \\
\hat{\Gamma}_{y} & =0 & & \text { on } y=0, \\
\hat{\Gamma}_{y} & =-\mathrm{i} \mu \hat{\eta}+\hat{F}_{3}(\eta, \Gamma) & & \text { on } y=1
\end{aligned}
$$

for the Fourier transform $\hat{\Gamma}(\mu, y)$ of $\Gamma(x, y)$, where $q=\sqrt{\mu^{2}+\lambda^{2}}$,

$$
\begin{aligned}
F_{2}(\eta, \Gamma) & =-\mathrm{i} \lambda \eta^{\star} \Gamma+\mathrm{i} \lambda y \Phi_{y}^{\star} \eta, \\
F^{\dagger}\left(u^{\dagger}\right) & =\xi^{\dagger}+\mathrm{i} \lambda\left(1+\eta^{\star}\right) \Gamma^{\dagger}-\mathrm{i} \lambda y \Phi_{y}^{\star} \eta^{\dagger}
\end{aligned}
$$


and $\xi^{\dagger}=-\xi_{1 x}^{\dagger}-\xi_{3 y}^{\dagger}$ for $\lambda=0$. On the other hand, substituting 31, 32, into 26, yields

$$
\begin{aligned}
& \left(\alpha_{0}+\varepsilon^{2}\right) \eta-\left.\Gamma_{x}\right|_{y=1}-\beta_{0} \eta_{x x}+h_{2}(\eta, \Gamma) \\
& =\omega^{\dagger}+\mathrm{i} \lambda \frac{\beta_{0}}{\left(1+\left(\eta_{x}^{\star}\right)^{2}\right)^{1 / 2}}\left(\eta^{\dagger}+\mathrm{i} \lambda \eta\right) \\
& \quad-\frac{\mathrm{i} \lambda}{1+\eta^{\star}} \int_{0}^{1} y \Phi_{y}^{\star}\left[\left(1+\eta^{\star}\right)\left(\Gamma^{\dagger}+\mathrm{i} \lambda \Gamma\right)-y \Phi_{y}^{\star}\left(\eta^{\dagger}+\mathrm{i} \lambda \eta\right)\right] \mathrm{d} y,
\end{aligned}
$$

which we write as

$$
g_{\varepsilon}(D, \lambda) \eta=\mathcal{N}\left(\eta, \Gamma, u^{\dagger}\right)
$$

where

$$
g_{\varepsilon}(\mu, \lambda)=\alpha_{0}+\varepsilon^{2}+\beta_{0} q^{2}-\frac{\mu^{2}}{q^{2}} q \operatorname{coth} q
$$

and

$$
\begin{aligned}
\mathcal{N}\left(\eta, \Gamma, u^{\dagger}\right) & \\
=\omega^{\dagger} & +\mathrm{i} \lambda \beta_{0} \frac{\eta^{\dagger}}{\left(1+\left(\eta_{x}^{\star}\right)^{2}\right)^{1 / 2}}+\left.\Gamma_{x}\right|_{y=1}-\mathcal{F}^{-1}\left[\frac{\mu^{2}}{q^{2}} q \operatorname{coth} q \hat{\eta}\right] \\
& -\beta_{0}\left(\eta_{x x}-\left[\frac{\eta_{x}}{\left(1+\left(\eta_{x}^{\star}\right)^{2}\right)^{3 / 2}}\right]_{x}\right)-\lambda^{2} \beta_{0}\left(\frac{1}{\left(1+\left(\eta_{x}^{\star}\right)^{2}\right)^{1 / 2}}-1\right) \eta \\
& -\frac{\mathrm{i} \lambda}{1+\eta^{\star}} \int_{0}^{1}\left[-\mathrm{i} \lambda y^{2}\left(\Phi_{y}^{\star}\right)^{2} \eta-y^{2}\left(\Phi_{y}^{\star}\right)^{2} \eta^{\dagger}+\mathrm{i} \lambda y \Phi_{y}^{\star} \Gamma+y \Phi_{y}^{\star} \Gamma^{\dagger}+\mathrm{i} \lambda y \Phi_{y}^{\star} \eta^{\star} \Gamma+y \Phi_{y}^{\star} \eta^{\star} \Gamma^{\dagger}\right] \mathrm{d} y \\
& -\int_{0}^{1}\left\{\Phi_{x}^{\star} \Gamma_{x}-\frac{\Phi_{y}^{\star} \Gamma_{y}}{\left(1+\eta^{\star}\right)^{2}}+\frac{\left(\Phi_{y}^{\star}\right)^{2} \eta}{\left(1+\eta^{\star}\right)^{3}}-\frac{y^{2}\left(\eta_{x}^{\star}\right)^{2} \Phi_{y}^{\star} \Gamma_{y}}{\left(1+\eta^{\star}\right)^{2}}-\frac{y^{2} \eta_{x}^{\star}\left(\Phi_{y}^{\star}\right)^{2} \eta_{x}}{\left(1+\eta^{\star}\right)^{2}}+\frac{y^{2} \eta_{x}^{\star}\left(\Phi_{y}^{\star}\right)^{2} \eta}{\left(1+\eta^{\star}\right)^{3}}\right. \\
& \left.+\left[y \Phi_{y}^{\star} \Gamma_{x}+y \Phi_{x}^{\star} \Gamma_{y}-\frac{2 y^{2} \eta_{x}^{\star} \Phi_{y}^{\star} \Gamma_{y}}{1+\eta^{\star}}-\frac{y^{2}\left(\Phi_{y}^{\star}\right)^{2} \eta_{x}}{1+\eta^{\star}}+\frac{y^{2}\left(\Phi_{y}^{\star}\right)^{2} \eta_{x}^{\star} \eta}{\left(1+\eta^{\star}\right)^{2}}\right]_{x}\right\} \mathrm{d} y .
\end{aligned}
$$

The next step is to express $\Gamma$ as a function of $\eta$. To this end we formulate the boundary-value problem (33)-35) as the integral equation

$$
\Gamma=\mathcal{G}_{1}\left(F_{1}(\eta, \Gamma), F_{2}(\eta, \Gamma), F_{3}(\eta, \Gamma), \eta\right)+\mathcal{G}_{2}\left(F^{\dagger}\left(u^{\dagger}\right)\right)
$$

where

$$
\begin{aligned}
\mathcal{G}_{1}\left(P_{1}, P_{2}, P_{3}, p\right) & =\mathcal{F}^{-1}\left[\int_{0}^{1}\left\{G(y, \tilde{y})\left(-\mathrm{i} \mu \hat{P}_{1}-\mathrm{i} \lambda \hat{P}_{2}\right)+G_{\tilde{y}}(y, \tilde{y}) \hat{P}_{3}\right\} \mathrm{d} \tilde{y}-\mathrm{i} \mu G(y, 1) \hat{p}\right], \\
\mathcal{G}_{2}(P) & =\mathcal{F}^{-1}\left[\int_{0}^{1} G(y, \tilde{y}) \hat{P} \mathrm{~d} \tilde{y}\right]
\end{aligned}
$$

and

$$
G(y, \tilde{y})= \begin{cases}\frac{\cosh (q y) \cosh (q(1-\tilde{y}))}{q \sinh q}, & 0 \leq y \leq \tilde{y} \leq 1, \\ \frac{\cosh (q \tilde{y}) \cosh (q(1-y))}{q \sinh q}, & 0 \leq \tilde{y} \leq y \leq 1 .\end{cases}
$$

The following proposition records the mapping properties of the integral operators appearing on the right hand side of (37); it is proved using estimates for $G(y, \tilde{y})$ similar to those given by Groves, Haragus \& Sun [14, Proposition 3.2]. 


\section{Proposition 3.1}

(i) The mapping $\left(P_{1}, P_{2}, P_{3}, p\right) \mapsto \mathcal{G}_{1}\left(P_{1}, P_{2}, P_{3}, p\right)$ defines a linear function

$$
\begin{cases}H^{1}(\Sigma) \times H^{1}(\Sigma) \times H^{1}(\Sigma) \times H^{2}(\mathbb{R}) \rightarrow H^{2}(\Sigma), & \lambda>0, \\ H_{\mathrm{e}}^{1}(\Sigma) \times H_{\mathrm{o}}^{1}(\Sigma) \times H_{\mathrm{o}}^{1}(\Sigma) \times H_{\mathrm{e}}^{2}(\mathbb{R}) \rightarrow H_{\star, \mathrm{o}}^{2}(\Sigma), & \lambda=0,\end{cases}
$$

which satisfies the estimate

$$
\begin{aligned}
& \left\|\nabla \mathcal{G}_{1}\right\|_{1}+\lambda\left\|\mathcal{G}_{1}\right\|_{1}+\lambda^{2}\left\|\mathcal{G}_{1}\right\|_{0} \\
& \quad \leq c\left(\left\|P_{1}\right\|_{1}+\left\|P_{2}\right\|_{1}+\left\|P_{3}\right\|_{1}+\lambda\left(\left\|P_{2}\right\|_{0}+\left\|P_{2}\right\|_{0}+\left\|P_{3}\right\|_{0}\right)+\left(1+\lambda^{2}\right)\|p\|_{0}+\left\|p_{x x}\right\|_{0}\right) .
\end{aligned}
$$

(ii) Suppose that $\lambda>0$. The mapping $P \mapsto \mathcal{G}_{2}(P)$ defines a linear function $L^{2}(\Sigma) \rightarrow$ $H^{2}(\Sigma)$ which satisfies the estimate

$$
\left\|\nabla \mathcal{G}_{2}\right\|_{1}+\lambda\left\|\mathcal{G}_{2}\right\|_{1}+\lambda^{2}\left\|\mathcal{G}_{2}\right\|_{0} \leq c\left(1+\lambda^{-1}\right)\|P\|_{0} .
$$

(iii) Suppose that $\lambda=0$ and $P=-P_{1 x}-P_{3 y}$, where $P_{1} \in H_{\mathrm{e}}^{1}(\Sigma)$ and $P_{3} \in \stackrel{\circ}{H}_{\mathrm{o}}^{1}(\Sigma)$. The mapping $\left(P_{1}, P_{3}\right) \mapsto \mathcal{G}_{2}(P)$ defines a linear function $H_{\mathrm{e}}^{1}(\Sigma) \times H_{\mathrm{o}}^{1}(\Sigma) \rightarrow H_{\star, \mathrm{o}}^{2}(\Sigma)$ which satisfies the estimate

$$
\left\|\nabla \mathcal{G}_{2}\right\|_{1} \leq c\left(\left\|P_{1}\right\|_{1}+\left\|P_{3}\right\|_{1}\right)
$$

\section{Theorem 3.2}

(i) Suppose that $\lambda>0$. For each sufficiently small value of $\varepsilon$ and each $\eta \in H^{2}(\mathbb{R})$, $F^{\dagger} \in L^{2}(\Sigma)$, equation (37) has a unique solution $\tilde{\Gamma}=\tilde{\Gamma}\left(\eta, u^{\dagger}\right) \in H^{2}(\Sigma)$ which satisfies the estimate

$$
\|\nabla \tilde{\Gamma}\|_{1}+\lambda\|\tilde{\Gamma}\|_{1}+\lambda^{2}\|\tilde{\Gamma}\|_{0} \leq c\left(\left(1+\lambda^{2}\right)\|\eta\|_{0}+\left\|\eta_{x x}\right\|_{0}+\left(1+\lambda^{-1}\right)\left\|F^{\dagger}\right\|_{0}\right) .
$$

(ii) Suppose that $\lambda=0$. For each sufficiently small value of $\varepsilon$ and each $\eta \in H_{\mathrm{e}}^{2}(\mathbb{R})$, $\xi_{1}^{\dagger} \in H_{\mathrm{e}}^{1}(\Sigma), \xi_{3}^{\dagger} \in \stackrel{H}{\mathrm{o}}_{\mathrm{o}}^{1}(\Sigma)$, equation (37) has a unique solution $\tilde{\Gamma}=\tilde{\Gamma}\left(\eta, \xi_{1}^{\dagger}, F_{2}^{\dagger}\right) \in H_{\star, \mathrm{o}}^{2}(\Sigma)$ which satisfies the estimate

$$
\|\nabla \tilde{\Gamma}\|_{1} \leq c\left(\|\eta\|_{2}+\left\|\xi_{1}^{\dagger}\right\|_{1}+\left\|\xi_{3}^{\dagger}\right\|_{1}\right) .
$$

Proof. It follows from the estimates

$$
\begin{aligned}
\left\|F_{1}\right\|_{1},\left\|F_{3}\right\|_{1} & \leq c \varepsilon\left(\|\eta\|_{1}+\left\|\eta_{x}\right\|_{1}+\|\nabla \Gamma\|_{1}\right) \\
& \leq c \varepsilon\left(\|\eta\|_{0}+\left\|\eta_{x x}\right\|_{0}+\|\nabla \Gamma\|_{1}\right), \\
\lambda\left\|F_{1}\right\|_{0}, \lambda\left\|F_{3}\right\|_{0} & \leq c \varepsilon\left(\lambda\|\eta\|_{0}+\lambda\left\|\eta_{x}\right\|_{0}+\lambda\|\nabla \Gamma\|_{0}\right) \\
& \leq c \varepsilon\left(\left(1+\lambda^{2}\right)\|\eta\|_{0}+\left\|\eta_{x x}\right\|_{0}+\lambda\|\nabla \Gamma\|_{0}\right), \\
\left\|F_{2}\right\|_{1} & \leq c \varepsilon\left(\lambda\|\eta\|_{1}+\lambda\|\Gamma\|_{1}\right) \\
& \leq c \varepsilon\left(\left(1+\lambda^{2}\right)\|\eta\|_{0}+\left\|\eta_{x x}\right\|_{0}+\lambda\|\Gamma\|_{1}\right), \\
\lambda\left\|F_{2}\right\|_{0} & \leq c\left(\lambda^{2}\|\eta\|_{0}+\lambda^{2}\|\Gamma\|_{0}\right)
\end{aligned}
$$


and Proposition 3.1(i) that

$$
\begin{aligned}
& \left\|\nabla \mathcal{G}_{1}(\eta, \Gamma)\right\|_{1}+\lambda\left\|\mathcal{G}_{1}(\eta, \Gamma)\right\|_{1}+\lambda^{2}\left\|\mathcal{G}_{1}(\eta, \Gamma)\right\|_{0} \\
& \quad \leq c\left(\varepsilon\left(\|\nabla \Gamma\|_{1}+\lambda\|\Gamma\|_{1}+\lambda^{2}\|\Gamma\|_{0}\right)+\left(1+\lambda^{2}\right)\|\eta\|_{0}+\left\|\eta_{x x}\right\|_{0}\right),
\end{aligned}
$$

in which $\mathcal{G}_{1}(\eta, \Gamma)$ is used an abbreviation for $\mathcal{G}_{1}\left(F_{1}(\eta, \Gamma), F_{2}(\eta, \Gamma), F_{3}(\eta, \Gamma), \eta\right)$. Using this estimate together with Proposition 3.1 (ii) or (iii) as appropriate and equipping $H^{2}(\Sigma)$ with the norm $\Gamma \mapsto\|\nabla \Gamma\|_{1}+\lambda\|\Gamma\|_{1}+\lambda^{2}\|\Phi\|_{0}$, one finds that (37) admits a unique solution which satisfies the stated estimate.

Substituting $\Gamma=\tilde{\Gamma}\left(\eta, u^{\dagger}\right)$ into $(36)$, we obtain a single equation for $\eta$, namely

$$
g_{\varepsilon}(D, \lambda) \eta=\tilde{\mathcal{N}}\left(\eta, u^{\dagger}\right)
$$

where

$$
\tilde{\mathcal{N}}\left(\eta, u^{\dagger}\right)=\mathcal{N}\left(\eta, \tilde{\Gamma}\left(\eta, u^{\dagger}\right), u^{\dagger}\right) .
$$

Finally, we record an estimate for $g_{\varepsilon}(\mu, \lambda)$ which is obtained by elementary calculus and use it to solve (38) for large values of $\lambda$, thus proving Theorem 2.2(i).

Proposition 3.3 For each fixed $\lambda^{\star}>0$ the quantity $g_{\varepsilon}(\mu, \lambda)$, which is even in both its arguments, satisfies the estimates

$$
c\left(\left(\mu-\mu_{0}\right)^{2}+\lambda^{2}+\varepsilon^{2}\right) \leq g_{\varepsilon}(\mu, \lambda) \leq \frac{1}{c}\left(\left(\mu-\mu_{0}\right)^{2}+\lambda^{2}+\varepsilon^{2}\right), \quad(\mu, \lambda) \in S
$$

and

$$
c\left(1+q^{2}+\varepsilon^{2}\right) \leq g_{\varepsilon}(\mu, \lambda) \leq \frac{1}{c}\left(1+q^{2}+\varepsilon^{2}\right), \quad(\mu, \lambda) \in[0, \infty)^{2} \backslash S,
$$

where $S=\left[\mu_{0}-\delta, \mu_{0}+\delta\right] \times\left[0, \lambda^{\star}\right]$.

Lemma 3.4 Choose $\lambda^{\star}>0$. For each sufficiently small value of $\varepsilon$, each $\lambda>\lambda^{\star}$ and each $u^{\dagger} \in X^{0}$, equation (38) has a unique solution $\eta=\eta\left(u^{\dagger}\right) \in H^{2}(\mathbb{R})$ which satisfies the estimate

$$
\left(1+\lambda^{2}\right)\|\eta\|_{0}+\left\|\eta_{x x}\right\|_{0} \leq c_{\lambda}\left\|u^{\dagger}\right\|_{X^{0}} .
$$

Proof. Write (38) as

$$
g_{\varepsilon}(D, \lambda) \eta-\tilde{\mathcal{N}}(\eta, 0)=\tilde{\mathcal{N}}\left(0, u^{\dagger}\right)
$$

and recall the estimates $g_{\varepsilon}(\mu, \lambda) \geq c\left(1+\mu^{2}+\lambda^{2}\right)$ (see Proposition 3.3) and

$$
\|\tilde{\mathcal{N}}(\eta, 0)\|_{0} \leq c \varepsilon\left(\left(1+\lambda^{2}\right)\|\eta\|_{0}+\left\|\eta_{x x}\right\|_{0}\right) .
$$

Equipping $H^{2}(\mathbb{R})$ with the norm $\eta \mapsto\left(1+\lambda^{2}\right)\|\eta\|_{0}+\left\|\eta_{x x}\right\|_{0}$, one finds that the operator

$$
g_{\varepsilon}(D, \lambda)-\tilde{\mathcal{N}}(\cdot, 0): L_{\mathrm{e}}^{2}(\mathbb{R}) \rightarrow H_{\mathrm{e}}^{2}(\mathbb{R})
$$

is invertible for each sufficiently small value of $\varepsilon$, and the result follows from this fact and the estimate

$$
\left\|\tilde{\mathcal{N}}\left(0, u^{\dagger}\right)\right\|_{0} \leq c_{\lambda}\left\|u^{\dagger}\right\|_{X^{0}} .
$$

In view of the previous lemma we now fix $\lambda^{\star}>0$ and henceforth suppose that $\lambda \leq \lambda^{\star}$. We write $(38)$ as

$$
g_{\varepsilon}(\mu, \lambda) \hat{\eta}_{1}=\chi \mathcal{F}\left[\tilde{\mathcal{N}}\left(\eta_{1}+\eta_{2}, u^{\dagger}\right)\right]
$$

and

$$
g_{\varepsilon}(\mu, \lambda) \hat{\eta}_{2}=(1-\chi) \mathcal{F}\left[\tilde{\mathcal{N}}\left(\eta_{1}+\eta_{2}, u^{\dagger}\right)\right],
$$

where $\hat{\eta}_{1}=\chi \hat{\eta}$ and $\hat{\eta}_{2}=(1-\chi) \hat{\eta}$. 
Lemma 3.5 The linear operator

$$
g_{\varepsilon}(D, \lambda)-(1-\chi(D)) \tilde{\mathcal{N}}(\cdot, 0):(1-\chi(D)) H^{2}(\mathbb{R}) \rightarrow(1-\chi(D)) L^{2}(\mathbb{R})
$$

is invertible for each sufficiently small value of $\varepsilon$.

Proof. This result follows from the estimates $g_{\varepsilon}(\mu, \lambda) \geq c\left(1+q^{2}\right)$ for ||$\mu\left|-\mu_{0}\right|>\delta$ (see Proposition 3.3) and

$$
\|\tilde{\mathcal{N}}(\eta, 0)\|_{0} \leq c \varepsilon\|\eta\|_{2}
$$

\section{Corollary 3.6}

(i) Suppose that $\lambda>0$. For each sufficiently small value of $\varepsilon$ and each $\eta_{1} \in \chi(D) L^{2}(\mathbb{R})$, $u^{\dagger} \in X^{0}$, equation (40) has a unique solution $\eta_{2}=\check{\eta}_{2}\left(\eta_{1}, u^{\dagger}\right) \in H^{2}(\mathbb{R})$ which satisfies the estimate

$$
\left\|\check{\eta}_{2}\right\|_{2} \leq c \varepsilon\left\|\eta_{1}\right\|_{0}+c_{\lambda}\left\|u^{\dagger}\right\|_{X^{0}}
$$

(ii) Suppose that $\lambda=0$. For each sufficiently small value of $\varepsilon$ and each $\eta_{1} \in \chi(D) L_{\mathrm{e}}^{2}(\mathbb{R})$, $u^{\dagger} \in X_{\mathrm{r}}^{0}$, equation (40) has a unique solution $\eta_{2}=\check{\eta}_{2}\left(\eta_{1}, u^{\dagger}\right) \in H_{\mathrm{e}}^{2}(\mathbb{R})$ which satisfies the estimate

$$
\left\|\check{\eta}_{2}\right\|_{2} \leq c\left(\varepsilon\left\|\eta_{1}\right\|_{0}+\left\|\omega^{\dagger}\right\|_{0}+\left\|\xi_{1}^{\dagger}\right\|_{1}+\left\|\xi_{3}^{\dagger}\right\|_{1}\right)
$$

Proof. Write (40) as

$$
g_{\varepsilon}(D, \lambda)\left(\eta_{2}\right)-(1-\chi(D)) \tilde{\mathcal{N}}\left(\eta_{2}, 0\right)=(1-\chi(D))\left(\tilde{\mathcal{N}}\left(\eta_{1}, 0\right)+\tilde{\mathcal{N}}\left(0, u^{\dagger}\right)\right)
$$

and use Lemma 3.5 and the estimates

$$
\left\|\tilde{\mathcal{N}}\left(\eta_{1}, 0\right)\right\|_{0} \leq c \varepsilon\left\|\eta_{1}\right\|_{0}, \quad\left\|\tilde{\mathcal{N}}\left(0, u^{\dagger}\right)\right\|_{0} \leq \begin{cases}c_{\lambda}\left\|u^{\dagger}\right\|_{X^{0}}, & \lambda>0 \\ c\left(\left\|\omega^{\dagger}\right\|_{0}+\left\|\xi_{1}^{\dagger}\right\|_{1}+\left\|\xi_{3}^{\dagger}\right\|_{1}\right), & \lambda=0\end{cases}
$$

Substituting $\check{\eta}_{2}=\check{\eta}_{2}\left(\eta_{1}, u^{\dagger}\right)$ into 39 , we obtain a single equation for $\eta_{1}$, namely

$$
g_{\varepsilon}(\mu, \lambda) \hat{\eta}_{1}=\chi \mathcal{F}\left[\check{\mathcal{N}}\left(\eta_{1}, u^{\dagger}\right)\right],
$$

where

$$
\begin{aligned}
\check{\mathcal{N}}\left(\eta_{1}, u^{\dagger}\right) & =\tilde{\mathcal{N}}\left(\eta_{1}+\check{\eta}_{2}\left(\eta_{1}, u^{\dagger}\right), u^{\dagger}\right) \\
& =\mathcal{N}\left(\eta_{1}+\check{\eta}_{2}\left(\eta_{1}, u^{\dagger}\right), \check{\Gamma}\left(\eta_{1}, u^{\dagger}\right), u^{\dagger}\right)
\end{aligned}
$$

and

$$
\check{\Gamma}\left(\eta_{1}, u^{\dagger}\right)=\tilde{\Gamma}\left(\eta_{1}+\check{\eta}_{2}\left(\eta_{1}, u^{\dagger}\right), u^{\dagger}\right)
$$




\subsection{Solution of the reduced equation for intermediate spectral values}

The next step is to derive more precise estimates for the functions introduced in the previous section. We expand $\eta^{\star}$ and $\Phi^{\star}$ as

$$
\eta^{\star}(x)=\eta_{1}^{\star}(x)+\eta_{2}^{\star}(x)+\eta_{\mathrm{r}}^{\star}(x), \quad \Phi^{\star}(x, y)=\Phi_{1}^{\star}(x, y)+\Phi_{2}^{\star}(x, y)+\Phi_{\mathrm{r}}^{\star}(x, y)
$$

and $\tilde{\Gamma}=\tilde{\Gamma}\left(\eta, u^{\dagger}\right)$ as

$$
\tilde{\Gamma}\left(\eta, u^{\dagger}\right)=\tilde{\Gamma}^{(1)}(\eta)+\tilde{\Gamma}^{(2)}(\eta)+\tilde{\Gamma}^{(3)}(\eta)+\tilde{\Gamma}^{\mathrm{r}}(\eta)+\tilde{\Gamma}^{\dagger}\left(u^{\dagger}\right) .
$$

Here $\eta_{1}^{\star}, \eta_{2}^{\star}, \eta_{\mathrm{r}}^{\star}$ and $\Phi_{1}^{\star}, \Phi_{2}^{\star}, \Phi_{\mathrm{r}}^{\star}$ are given in the Appendix (equations $(73)-(76)$ ),

$$
\begin{aligned}
& \tilde{\Gamma}^{(1)}(\eta)=\mathcal{G}_{1}(0,0, \eta), \\
& \tilde{\Gamma}^{(2)}(\eta)=\mathcal{G}_{1}\left(F_{1}^{(2)}(\eta), F_{2}^{(2)}(\eta), F_{3}^{(2)}(\eta), 0\right), \\
& \tilde{\Gamma}^{(3)}(\eta)=\mathcal{G}_{1}\left(F_{1}^{(3)}(\eta), F_{2}^{(3)}(\eta), F_{3}^{(3)}(\eta), 0\right)
\end{aligned}
$$

with

$$
\begin{aligned}
& F_{1}^{(2)}(\eta)=-\eta_{1}^{\star} \tilde{\Gamma}_{x}^{(1)}(\eta)-\Phi_{1 x}^{\star} \eta+y \Phi_{1 y}^{\star} \eta_{x}+y \eta_{1 x}^{\star} \tilde{\Gamma}_{y}^{(1)}(\eta), \\
& F_{2}^{(2)}(\eta)=-\mathrm{i} \lambda \eta_{1}^{\star} \tilde{\Gamma}^{(1)}(\eta)+\mathrm{i} \lambda y \Phi_{1 y}^{\star} \eta \\
& F_{3}^{(2)}(\eta)=y \eta_{1 x}^{\star} \tilde{\Gamma}_{x}^{(1)}(\eta)+y \Phi_{1 x}^{\star} \eta_{x}+\eta_{1}^{\star} \tilde{\Gamma}_{y}^{(1)}(\eta)+\Phi_{1 y}^{\star} \eta \\
& F_{1}^{(3)}(\eta)=-\eta_{1}^{\star} \tilde{\Gamma}_{x}^{(2)}(\eta)-\eta_{2}^{\star} \tilde{\Gamma}_{x}^{(1)}(\eta)-\Phi_{2 x}^{\star} \eta+y \Phi_{2 y}^{\star} \eta_{x}+y \eta_{1 x}^{\star} \tilde{\Phi}_{y}^{(2)}(\eta)+y \eta_{2 x}^{\star} \tilde{\Gamma}_{y}^{(1)}(\eta), \\
& F_{2}^{(3)}(\eta)=-\mathrm{i} \lambda \eta_{1}^{\star} \tilde{\Gamma}^{(2)}(\eta)-\mathrm{i} \lambda \eta_{2}^{\star} \tilde{\Gamma}^{(1)}(\eta)+\mathrm{i} \lambda y \Phi_{2 y}^{\star} \eta \\
& F_{3}^{(3)}(\eta)=y \eta_{1 x}^{\star} \tilde{\Gamma}_{x}^{(2)}(\eta)+y \eta_{2 x}^{\star} \tilde{\Gamma}_{x}^{(1)}(\eta)+y \Phi_{2 x}^{\star} \eta_{x}+\eta_{1}^{\star} \tilde{\Gamma}_{y}^{(2)}(\eta)+\eta_{2}^{\star} \tilde{\Gamma}_{y}^{(1)}(\eta)+\Phi_{2 y}^{\star} \eta \\
& \quad-\left(\eta_{1}^{\star}\right)^{2} \tilde{\Gamma}_{y}^{(1)}(\eta)-2 \Phi_{1 y}^{\star} \eta_{1}^{\star} \eta-y^{2}\left(\eta_{1 x}^{\star}\right)^{2} \tilde{\Gamma}_{y}^{(1)}(\eta)-2 y^{2} \eta_{1 x}^{\star} \Phi_{1 y}^{\star} \eta_{x},
\end{aligned}
$$

and $\tilde{\Gamma}^{\mathrm{r}}(\eta), \tilde{\Gamma}^{\dagger}\left(u^{\dagger}\right)$ are determined implicitly by

$$
\tilde{\Gamma}^{\mathrm{r}}(\eta)=\mathcal{G}_{1}\left(F_{1}^{\mathrm{r}}(\eta), F_{2}^{\mathrm{r}}(\eta), F_{3}^{\mathrm{r}}(\eta), 0\right)
$$

with

$$
F_{j}^{\mathrm{r}}(\eta)=F_{j}\left(\eta, \tilde{\Gamma}^{(1)}(\eta)+\tilde{\Gamma}^{(2)}(\eta)+\tilde{\Gamma}^{(3)}(\eta)+\tilde{\Gamma}^{\mathrm{r}}(\eta)\right)-F_{j}^{(2)}(\eta)-F_{j}^{(3)}(\eta), \quad j=1,2,3,
$$

and

$$
\tilde{\Gamma}^{\dagger}=\mathcal{G}_{1}\left(F_{1}\left(0, \tilde{\Gamma}^{\dagger}\right), F_{2}\left(0, \tilde{\Gamma}^{\dagger}\right), F_{3}\left(0, \tilde{\Gamma}^{\dagger}\right), 0\right)+\mathcal{G}_{2}\left(F^{\dagger}\left(u^{\dagger}\right)\right) .
$$

Lemma 3.7 The functions $\tilde{\Gamma}^{(1)}, \tilde{\Gamma}^{(2)}, \tilde{\Gamma}^{(3)}, \tilde{\Gamma}^{\mathrm{r}}$ and $\tilde{\Gamma}^{\dagger}$ satisfy the estimates

$$
\begin{aligned}
&\left\|\nabla \tilde{\Gamma}^{(j)}(\eta)\right\|_{1}+\lambda\left\|\tilde{\Gamma}^{(j)}(\eta)\right\|_{1}+\lambda^{2}\left\|\tilde{\Gamma}^{(j)}(\eta)\right\|_{0} \leq c \varepsilon^{j-1}\|\eta\|_{2}, \quad j=1,2,3, \\
&\left\|\nabla \tilde{\Gamma}^{\mathrm{r}}(\eta)\right\|_{1}+\lambda\left\|\tilde{\Gamma}^{\mathrm{r}}(\eta)\right\|_{1}+\lambda^{2}\left\|\tilde{\Gamma}^{\mathrm{r}}(\eta)\right\|_{0} \leq c \varepsilon^{3}\|\eta\|_{2}, \\
&\left\|\nabla \tilde{\Gamma}^{\dagger}\right\|_{1}+\lambda\left\|\tilde{\Gamma}^{\dagger}\right\|_{1}+\lambda^{2}\left\|\tilde{\Gamma}^{\dagger}\right\|_{0} \leq \begin{cases}c_{\lambda}\left\|u^{\dagger}\right\|_{X^{0}}, & \lambda>0, \\
c\left(\left\|\omega^{\dagger}\right\|_{0}+\left\|\xi_{1}^{\dagger}\right\|_{1}+\left\|\xi_{3}^{\dagger}\right\|_{1}\right), & \lambda=0 .\end{cases}
\end{aligned}
$$


We correspondingly write

$$
\tilde{\mathcal{N}}\left(\eta, u^{\dagger}\right)=\tilde{\mathcal{N}}^{(2)}(\eta)+\tilde{\mathcal{N}}^{(3)}(\eta)+\tilde{\mathcal{N}}^{\mathrm{r}}(\eta)+\tilde{\mathcal{N}}^{\dagger}\left(u^{\dagger}\right)
$$

where

$$
\begin{aligned}
\tilde{\mathcal{N}}^{(2)}(\eta)= & \left.\tilde{\Gamma}_{x}^{(2)}(\eta)\right|_{y=1}-\mathrm{i} \lambda \int_{0}^{1} y \Phi_{1 y}^{\star} \mathrm{i} \lambda \tilde{\Gamma}^{(1)}(\eta) \mathrm{d} y \\
& -\int_{0}^{1}\left\{\Phi_{1 x}^{\star} \tilde{\Gamma}_{x}^{(1)}(\eta)-\Phi_{1 y}^{\star} \tilde{\Gamma}_{y}^{(1)}(\eta)+\left[y \Phi_{1 y}^{\star} \tilde{\Gamma}_{x}^{(1)}(\eta)+y \Phi_{1 x}^{\star} \tilde{\Gamma}_{y}^{(1)}(\eta)\right]_{x}\right\} \mathrm{d} y, \\
\tilde{\mathcal{N}}^{(3)}(\eta)= & \left.\tilde{\Gamma}_{x}^{(3)}(\eta)\right|_{y=1}-\frac{3 \beta_{0}}{2}\left[\left(\eta_{1 x}^{\star}\right)^{2} \eta_{x}\right]_{x}+\frac{\lambda^{2} \beta_{0}}{2} \eta_{1 x}^{\star 2} \eta \\
& -\mathrm{i} \lambda \int_{0}^{1}\left\{-\mathrm{i} \lambda y^{2}\left(\Phi_{1 y}^{\star}\right)^{2} \eta+\mathrm{i} \lambda y \Phi_{1 y}^{\star} \tilde{\Gamma}^{(2)}(\eta)-\mathrm{i} \lambda y \Phi_{2 y}^{\star} \tilde{\Gamma}^{(1)}(\eta)\right\} \mathrm{d} y \\
& -\int_{0}^{1}\left\{\Phi_{1 x}^{\star} \tilde{\Gamma}_{x}^{(2)}(\eta)-\Phi_{1 y}^{\star} \tilde{\Gamma}_{y}^{(2)}(\eta)+2 \Phi_{1 y}^{\star} \eta_{1}^{\star} \tilde{\Gamma}_{y}^{(1)}(\eta)+\left(\Phi_{1 y}^{\star}\right)^{2} \eta\right\} \mathrm{d} y \\
& -\int_{0}^{1}\left[y \Phi_{1 y}^{\star} \tilde{\Gamma}_{x}^{(2)}(\eta)+y \Phi_{1 x}^{\star} \tilde{\Gamma}_{y}^{(2)}(\eta)-2 y^{2} \eta_{1 x}^{\star} \Phi_{1 y}^{\star} \tilde{\Gamma}_{y}^{(1)}(\eta)-y^{2}\left(\Phi_{1 y}^{\star}\right)^{2} \eta_{x}\right]_{x} \mathrm{~d} y \\
& -\int_{0}^{1}\left\{\Phi_{2 x}^{\star} \tilde{\Gamma}_{x}^{(1)}(\eta)-\Phi_{2 y}^{\star} \tilde{\Gamma}_{y}^{(1)}(\eta)+\left[y \Phi_{2 y}^{\star} \tilde{\Gamma}_{x}^{(1)}(\eta)+y \Phi_{2 x}^{\star} \tilde{\Gamma}_{y}^{(1)}(\eta)\right]_{x}\right\} \mathrm{d} y, \quad \\
& \left.\tilde{\mathcal{N}}^{(\eta}, 0\right)-\tilde{\mathcal{N}}^{(2)}(\eta)-\tilde{\mathcal{N}}^{(3)}(\eta), \\
\tilde{\mathcal{N}}(\eta)= & \tilde{\mathcal{N}}\left(0, u^{\dagger}\right) .
\end{aligned}
$$

Lemma 3.8 The functions $\tilde{\mathcal{N}}^{(2)}, \tilde{\mathcal{N}}^{(3)}, \tilde{\mathcal{N}}^{\mathrm{r}}$ and $\tilde{\mathcal{N}}^{\dagger}$ satisfy the estimates

$$
\begin{aligned}
&\left\|\tilde{\mathcal{N}}^{(j)}(\eta)\right\|_{0} \leq c \varepsilon^{j-1}\|\eta\|_{2}, \quad j=2,3, \\
&\left\|\tilde{\mathcal{N}}^{\mathrm{r}}(\eta)\right\|_{0} \leq c \varepsilon^{3}\|\eta\|_{2}, \\
&\left\|\tilde{\mathcal{N}}^{\dagger}\left(u^{\dagger}\right)\right\|_{0} \leq \begin{cases}c_{\lambda}\left\|u^{\dagger}\right\|_{X^{0}}, & \lambda>0, \\
c\left(\left\|\omega^{\dagger}\right\|_{0}+\left\|\xi_{1}^{\dagger}\right\|_{1}+\left\|\xi_{3}^{\dagger}\right\|_{1}\right), & \lambda=0 .\end{cases}
\end{aligned}
$$

Similarly, we expand $\check{\eta}_{2}=\check{\eta}_{2}\left(\eta_{1}, u^{\dagger}\right)$ as

$$
\check{\eta}_{2}\left(\eta_{1}, u^{\dagger}\right)=\check{\eta}_{2}^{(2)}\left(\eta_{1}\right)+\check{\eta}_{2}^{\mathrm{r}}\left(\eta_{1}\right)+\check{\eta}_{2}^{\dagger}\left(u^{\dagger}\right)
$$

where

$$
\mathcal{F}\left[\check{\eta}_{2}^{(2)}\right]=g_{\varepsilon}(\mu, \lambda)^{-1}(1-\chi) \mathcal{F}\left[\tilde{\mathcal{N}}^{(2)}\left(\eta_{1}\right)\right]
$$

and $\check{\eta}_{2}^{\mathrm{r}}, \check{\eta}_{2}^{\dagger}$ are determined implicitly by

$$
\begin{aligned}
& \mathcal{F}\left[\check{\eta}_{2}^{\mathrm{r}}\right]=g_{\varepsilon}(\mu, \lambda)^{-1}(1-\chi) \mathcal{F}\left[\tilde{\mathcal{N}}^{(3)}\left(\eta_{1}\right)+\tilde{\mathcal{N}}^{\mathrm{r}}\left(\eta_{1}\right)+\tilde{\mathcal{N}}\left(\check{\eta}_{2}^{(2)}+\check{\eta}_{2}^{\mathrm{r}}, 0\right)\right] \\
& \mathcal{F}\left[\check{\eta}_{2}^{\dagger}\right]=g_{\varepsilon}(\mu, \lambda)^{-1}(1-\chi) \mathcal{F}\left[\tilde{\mathcal{N}}\left(\check{\eta}_{2}^{\dagger}, u^{\dagger}\right)\right]
\end{aligned}
$$


and $\check{\Gamma}=\check{\Gamma}\left(\eta_{1}, u^{\dagger}\right)$ as

$$
\check{\Gamma}\left(\eta_{1}, u^{\dagger}\right)=\check{\Gamma}^{(1)}\left(\eta_{1}\right)+\check{\Gamma}^{(2)}\left(\eta_{1}\right)+\check{\Gamma}^{(3)}\left(\eta_{1}\right)+\check{\Gamma}^{\mathrm{r}}\left(\eta_{1}\right)+\check{\Gamma}^{\dagger}\left(u^{\dagger}\right),
$$

where

$$
\begin{aligned}
\check{\Gamma}^{(1)} & =\tilde{\Gamma}^{(1)}\left(\eta_{1}\right), \\
\check{\Gamma}^{(2)} & =\tilde{\Gamma}^{(2)}\left(\eta_{1}\right)+\tilde{\Gamma}^{(1)}\left(\check{\eta}_{2}^{(2)}\left(\eta_{1}\right)\right), \\
\check{\Gamma}^{(3)} & =\tilde{\Gamma}^{(3)}\left(\eta_{1}\right)+\tilde{\Gamma}^{(2)}\left(\check{\eta}_{2}^{(2)}\left(\eta_{1}\right)\right)+\tilde{\Gamma}^{(1)}\left(\check{\eta}_{2}^{\mathrm{r}}\left(\eta_{1}\right)\right), \\
\check{\Gamma}^{\mathrm{r}} & =\tilde{\Gamma}^{\mathrm{r}}\left(\eta_{1}+\check{\eta}_{2}^{(2)}\left(\eta_{1}\right)+\check{\eta}_{2}^{\mathrm{r}}\left(\eta_{1}\right)\right)+\tilde{\Gamma}^{(3)}\left(\check{\eta}_{2}^{(2)}\left(\eta_{1}\right)+\check{\eta}_{2}^{\mathrm{r}}\left(\eta_{1}\right)\right)+\tilde{\Gamma}^{(2)}\left(\check{\eta}_{2}^{\mathrm{r}}\left(\eta_{1}\right)\right), \\
\check{\Gamma}^{\dagger} & =\tilde{\Gamma}\left(\check{\eta}_{2}^{\dagger}\left(u^{\dagger}\right), u^{\dagger}\right) .
\end{aligned}
$$

\section{Proposition 3.9}

(i) The functions $\check{\eta}_{2}^{(2)}, \check{\eta}_{2}^{\mathrm{r}}$ and $\check{\eta}_{2}^{\dagger}$ satisfy the estimates

$$
\left\|\check{\eta}_{2}^{(2)}\right\|_{2} \leq c \varepsilon\left\|\eta_{1}\right\|_{0}, \quad\left\|\check{\eta}_{2}^{\mathrm{r}}\right\|_{2} \leq c \varepsilon^{2}\left\|\eta_{1}\right\|_{0}, \quad\left\|\check{\eta}_{2}^{\dagger}\right\|_{2} \leq \begin{cases}c_{\lambda}\left\|u^{\dagger}\right\|_{X^{0}}, & \lambda>0 \\ c\left(\left\|\omega^{\dagger}\right\|_{0}+\left\|\xi_{1}^{\dagger}\right\|_{1}+\left\|\xi_{3}^{\dagger}\right\|_{1}\right), & \lambda=0 .\end{cases}
$$

(ii) The functions $\check{\Gamma}^{(1)}, \check{\Gamma}^{(2)}, \check{\Gamma}^{(3)}, \check{\Gamma}^{\mathrm{r}}$ and $\check{\Gamma}^{\dagger}$ satisfy the estimates

$$
\begin{aligned}
&\left\|\nabla \check{\Gamma}^{(j)}\right\|_{1}+\lambda\left\|\check{\Gamma}^{(j)}\right\|_{0} \leq c \varepsilon^{j-1}\left\|\eta_{1}\right\|_{0}, \quad j=1,2,3, \\
&\left\|\nabla \check{\Gamma}^{\mathrm{r}}\right\|_{1}+\lambda\left\|\check{\Gamma}^{\mathrm{r}}\right\|_{0} \leq c \varepsilon^{3}\left\|\eta_{1}\right\|_{0}, \\
&\left\|\nabla \check{\Gamma}^{\dagger}\right\|_{1}+\lambda\left\|\check{\Gamma}^{\dagger}\right\|_{0} \leq \begin{cases}c_{\lambda}\left\|u^{\dagger}\right\|_{X^{0}}, & \lambda>0 \\
c\left(\left\|\omega^{\dagger}\right\|_{0}+\left\|\xi_{1}^{\dagger}\right\|_{1}+\left\|\xi_{3}^{\dagger}\right\|_{1}\right), & \lambda=0 .\end{cases}
\end{aligned}
$$

Finally, we write

$$
\check{\mathcal{N}}\left(\eta_{1}, u^{\dagger}\right)=\check{\mathcal{N}}^{(2)}\left(\eta_{1}\right)+\check{\mathcal{N}}^{(3)}\left(\eta_{1}\right)+\check{\mathcal{N}}^{\mathrm{r}}\left(\eta_{1}\right)+\check{\mathcal{N}}^{\dagger}\left(u^{\dagger}\right)
$$

where

$$
\begin{aligned}
\check{\mathcal{N}}^{(2)}\left(\eta_{1}\right) & =\tilde{\mathcal{N}}^{(2)}\left(\eta_{1}\right), \\
\check{\mathcal{N}}^{(3)}\left(\eta_{1}\right) & =\tilde{\mathcal{N}}^{(3)}\left(\eta_{1}\right)+\tilde{\mathcal{N}}^{(2)}\left(\check{\eta}_{2}^{(2)}\left(\eta_{1}\right)\right), \\
\check{\mathcal{N}}^{\mathrm{r}}\left(\eta_{1}\right) & =\tilde{\mathcal{N}}^{\mathrm{r}}\left(\eta_{1}+\check{\eta}_{2}^{(2)}\left(\eta_{1}\right)+\check{\eta}_{2}^{\mathrm{r}}\left(\eta_{1}\right)\right)+\tilde{\mathcal{N}}^{(3)}\left(\check{\eta}_{2}^{(2)}\left(\eta_{1}\right)+\check{\eta}_{2}^{\mathrm{r}}\left(\eta_{1}\right)\right)+\tilde{\mathcal{N}}^{(2)}\left(\check{\eta}_{2}^{\mathrm{r}}\left(\eta_{1}\right)\right), \\
\check{\mathcal{N}}^{\dagger}\left(u^{\dagger}\right) & =\tilde{\mathcal{N}}\left(\check{\eta}_{2}^{\dagger}\left(u^{\dagger}\right), u^{\dagger}\right) .
\end{aligned}
$$

Proposition 3.10 The functions $\check{\mathcal{N}}^{(2)}, \check{\mathcal{N}}^{(3)}, \check{\mathcal{N}}^{\mathrm{r}}$ and $\check{\mathcal{N}}^{\dagger}$ satisfy the estimates

$$
\begin{aligned}
\left\|\check{\mathcal{N}}^{(j)}\left(\eta_{1}\right)\right\|_{0} & \leq c \varepsilon^{j-1}\left\|\eta_{1}\right\|_{0}, \quad j=2,3, \\
\left\|\check{\mathcal{N}}^{\mathrm{r}}\left(\eta_{1}\right)\right\|_{0} & \leq c \varepsilon^{3}\left\|\eta_{1}\right\|_{0}, \\
\left\|\check{\mathcal{N}}^{\dagger}\left(u^{\dagger}\right)\right\|_{0} & \leq \begin{cases}c_{\lambda}\left\|u^{\dagger}\right\|_{X^{0}}, & \lambda>0, \\
c\left(\left\|\omega^{\dagger}\right\|_{0}+\left\|\xi_{1}^{\dagger}\right\|_{1}+\left\|\xi_{3}^{\dagger}\right\|_{1}\right), & \lambda=0 .\end{cases}
\end{aligned}
$$


Observing that $\chi(D) \check{N}^{(2)}(\eta)=0$ for all $\eta_{1} \in \chi(D) L^{2}(\mathbb{R})$, we find that the reduced equation (41) may be written as

$$
g_{\varepsilon}(\mu, \lambda) \hat{\eta}_{1}=\chi \mathcal{F}\left[\check{\mathcal{N}}^{(3)}\left(\eta_{1}\right)+\check{\mathcal{N}}^{\mathrm{r}}\left(\eta_{1}\right)+\check{\mathcal{N}}^{\dagger}\left(u^{\dagger}\right)\right]
$$

Lemma 3.11 There exists a constant $k_{\max }>0$ such that the linear operator

$$
g_{\varepsilon}(D, \lambda)-\chi(D)\left(\check{\mathcal{N}}^{(3)}(\cdot)+\check{\mathcal{N}}^{\mathrm{r}}(\cdot)\right): \chi(D) L^{2}(\mathbb{R}) \rightarrow \chi(D) L^{2}(\mathbb{R})
$$

is invertible for each $\lambda \in\left[\varepsilon k_{\max }, \lambda^{\star}\right]$.

Proof. Write $\lambda=\varepsilon k$ and observe that

$$
g_{\varepsilon}(\mu, \lambda) \geq c \varepsilon^{2}\left(1+k_{\max }^{2}\right)
$$

(see Proposition 3.3, while

$$
\left\|\check{\mathcal{N}}^{(3)}\left(\eta_{1}\right)+\check{\mathcal{N}}^{\mathrm{r}}\left(\eta_{1}\right)\right\|_{0} \leq c \varepsilon^{2}\left\|\eta_{1}\right\|_{2}
$$

It follows that the given operator is invertible for sufficiently large values of $k$.

Corollary 3.12 For each $\lambda \in\left[\varepsilon k_{\max }, \lambda^{\star}\right]$ and each $u \in X^{0}$, equation $(48)$ has a unique solution $\eta_{1} \in \chi(D) L^{2}(\mathbb{R})$ which satisfies the estimate

$$
\left\|\eta_{1}\right\|_{0} \leq c_{\lambda}\left\|u^{\dagger}\right\|_{X^{0}}
$$

In view of the previous corollary we now write $\lambda=\varepsilon k$ and henceforth suppose that $k \leq k_{\max }$.

\subsection{Calculation of the leading-order terms in the reduced equation}

In this section we calculate the leading-order terms in the quantity $\mathcal{N}^{(3)}\left(\eta_{1}\right)$ appearing in the reduced equation (48), writing

$$
\eta_{1}(x)=\frac{1}{2} \varepsilon \zeta(\varepsilon x) \mathrm{e}^{\mathrm{i} \mu_{0} x}+\frac{1}{2} \varepsilon \overline{\zeta(\varepsilon x)} \mathrm{e}^{-\mathrm{i} \mu_{0} x}
$$

where $\zeta \in \chi_{0}(\varepsilon D) L^{2}(\mathbb{R})$. For this purpose we first perform the corresponding calculation for $\check{\mathcal{N}}^{(2)}\left(\eta_{1}\right)$ using the formula

$$
\begin{aligned}
\check{\mathcal{N}}^{(2)}\left(\eta_{1}\right)=\left.\tilde{\Gamma}_{x}^{(2)}\left(\eta_{1}\right)\right|_{y=1}+k \varepsilon \int_{0}^{1} y \Phi_{1 y}^{\star} k \varepsilon \check{\Gamma}^{(1)} \mathrm{d} y \\
\quad-\int_{0}^{1}\left\{\Phi_{1 x}^{\star} \check{\Gamma}_{x}^{(1)}-\Phi_{1 y}^{\star} \check{\Gamma}_{y}^{(1)}+\left[y \Phi_{1 y}^{\star} \check{\Gamma}_{x}^{(1)}+y \Phi_{1 x}^{\star} \check{\Gamma}_{y}^{(1)}\right]_{x}\right\} \mathrm{d} y
\end{aligned}
$$

(see equation (46) together with

$$
\check{\Gamma}^{(1)}=\mathcal{F}^{-1}\left[-\mathrm{i} \mu G(y, 1) \hat{\eta}_{1}\right]
$$

and

$$
\tilde{\Gamma}^{(2)}\left(\eta_{1}\right)=\mathcal{F}^{-1}\left[\int_{0}^{1}\left\{G(y, \tilde{y})\left(-\mathrm{i} \mu \mathcal{F}\left[\check{F}_{1}^{(2)}\right]-\mathrm{i} k \varepsilon \mathcal{F}\left[\check{F}_{2}^{(2)}\right]\right)+G_{\tilde{y}}(y, \tilde{y}) \mathcal{F}\left[\check{F}_{3}^{(2)}\right]\right\} \mathrm{d} \tilde{y}\right]
$$


where

$$
\begin{aligned}
& \check{F}_{1}^{(2)}=-\eta_{1}^{\star} \check{\Gamma}_{x}^{(1)}-\Phi_{1 x}^{\star} \eta_{1}+y \Phi_{1 y}^{\star} \eta_{1 x}+y \eta_{1 x}^{\star} \check{\Gamma}_{y}^{(1)}, \\
& \check{F}_{2}^{(2)}=-\mathrm{i} k \varepsilon \eta_{1}^{\star} \check{\Phi}^{(1)}+\mathrm{i} k \varepsilon y \Phi_{1 y}^{\star} \eta_{1}, \\
& \check{F}_{3}^{(2)}=y \eta_{1 x}^{\star} \check{\Gamma}_{x}^{(1)}+y \Phi_{1 x}^{\star} \eta_{1 x}+\eta_{1}^{\star} \check{\Gamma}_{y}^{(1)}+\Phi_{1 y}^{\star} \eta_{1} .
\end{aligned}
$$

At various stages of the calculation terms arise of the form $G(y, 1) \hat{p}$ and $G(y, \tilde{y}) \hat{P}$, where $\hat{p}$ and $\hat{P}$ have compact support. We decompose such terms into a leading-order part and a higher-order remainder term using the following lemma.

\section{Lemma 3.13}

(i) Suppose that $\underline{\mu}>0$. The Green's function $G$ admits the decomposition

$$
G(y, \tilde{y})=G(y, \tilde{y} ; \underline{\mu})+R(y, \tilde{y})
$$

where

$$
G(y, \tilde{y} ; \underline{\mu})=\left\{\begin{array}{ll}
\frac{\cosh (\underline{\mu} y) \cosh (\underline{\mu}(1-\tilde{y}))}{\underline{\mu} \sinh \underline{\mu}}, & 0 \leq y \leq \tilde{y} \leq 1, \\
\frac{\cosh (\underline{\mu} \tilde{y}) \cosh (\underline{\mu}(1-y))}{\underline{\mu} \sinh \underline{\mu}}, & 0 \leq \tilde{y} \leq y \leq 1,
\end{array} .\right.
$$

and $R$ satisfies the estimates

$$
\left|\partial_{y}^{m_{1}} \partial_{\tilde{y}}^{m_{2}} R(y, \tilde{y})\right| \leq c\left(|\mu-\underline{\mu}|+\varepsilon^{2} k^{2}\right), \quad m_{1}, m_{2}=0,1,2, \ldots,
$$

uniformly over $y, \tilde{y}$ in $[0,1]$ and $q$ in each fixed interval $\left[q_{\min }, q_{\max }\right]$ containing $\underline{\mu}$.

(ii) The Green's function $G$ admits the expansion

$$
G(y, \tilde{y})=G(y, \tilde{y} ; 0)+R(y, \tilde{y})
$$

where

$$
G(y, \tilde{y} ; 0)=\frac{1}{q^{2}}
$$

and $R$ satisfies the estimate

$$
|R(y, \tilde{y})| \leq c
$$

uniformly over $y, \tilde{y}$ in $[0,1]$ and $q$ in each fixed interval $\left[0, q_{\max }\right]$.

The next proposition is proved by applying Lemma 3.13(i) with $\underline{\mu}= \pm \mu_{0}$ (and $q_{\text {min }}=\mu_{0}-\delta$, $\left.q_{\max }=\left(\left(\mu_{0}+\delta\right)^{2}+\lambda^{\star 2}\right)^{1 / 2}\right)$ to equation 50).

Proposition 3.14 The function $\check{\Gamma}^{(1)}$ is given by the formula

$$
\check{\Gamma}^{(1)}=-\mathrm{i} \frac{\cosh \left(\mu_{0} y\right)}{2 \sinh \mu_{0}}\left(\varepsilon \zeta(\varepsilon x) \mathrm{e}^{\mathrm{i} \mu_{0} x}-\varepsilon \overline{\zeta(\varepsilon x)} \mathrm{e}^{-\mathrm{i} \mu_{0} x}\right)+R_{2},
$$

where the symbol $R_{j}$ denotes a quantity which satisfies the estimate

$$
\left\|R_{j}\right\|_{j} \leq c \varepsilon^{3 / 2}\|\zeta\|_{1}
$$


Substituting this formula into equations (52)-(54) and approximating $\zeta^{\star}$ with

$$
\zeta_{\delta}^{\star}:=\chi_{0}(\varepsilon D) \zeta^{\star}
$$

by means of the estimate

$$
\varepsilon\left\|\zeta(\varepsilon \cdot)-\zeta_{\delta}(\varepsilon \cdot)\right\|_{\infty} \leq \int_{-\infty}^{\infty}\left|\hat{\zeta}^{\star}\left(\frac{\mu}{\varepsilon}\right)-\hat{\zeta}_{\delta}^{\star}\left(\frac{\mu}{\varepsilon}\right)\right| \mathrm{d} \mu=\int_{|\mu| \geq \delta}\left|\hat{\zeta}^{\star}\left(\frac{\mu}{\varepsilon}\right)\right| \mathrm{d} \mu=O\left(\varepsilon^{n}\right), \quad n>1,
$$

( $\zeta$ is a Schwartz-class function) yields the following result.

Corollary 3.15 The functions $\check{F}_{1}^{(2)}, \check{F}_{2}^{(2)}$ and $\check{F}_{3}^{(2)}$ are given by the formulae

$$
\begin{aligned}
& \check{F}_{1}^{(2)}= \varepsilon^{2} \frac{\mu_{0}\left(y \mu_{0} \sinh \left(\mu_{0}\right)-\cosh \left(\mu_{0} y\right)\right)}{\sinh \left(\mu_{0}\right)} \zeta_{\delta}^{\star}(\varepsilon x)\left(\frac{1}{2} \zeta(\varepsilon x) e^{2 \mathrm{i} \mu_{0} x}+\frac{1}{2} \overline{\zeta(\varepsilon x)} e^{-2 \mathrm{i} \mu_{0} x}\right) \\
&-\varepsilon^{2} \zeta_{\delta}^{\star}(\varepsilon x) \frac{\mu_{0}\left(\mu_{0} y \sinh \left(\mu_{0} y\right)+\cosh \left(\mu_{0} y\right)\right)}{\sinh \left(\mu_{0}\right)} \frac{1}{2}(\zeta(\varepsilon x)+\overline{\zeta(\varepsilon x)})+\varepsilon R_{1}, \\
& \check{F}_{2}^{(2)}=\varepsilon^{3} k \zeta_{\delta}^{\star}(\varepsilon x) \frac{y \mu_{0} \sinh \left(\mu_{0} y\right)-\cosh \left(\mu_{0} y\right)}{\sinh \mu_{0}}\left(\frac{1}{4} \zeta(\varepsilon x) e^{2 \mathrm{i} \mu_{0} x}-\frac{1}{4} \overline{\zeta(\varepsilon x)} e^{-2 \mathrm{i} \mu_{0} x}\right) \\
& \quad-\varepsilon^{3} k \zeta_{\delta}^{\star}(\varepsilon x) \frac{y \mu_{0} \sinh \left(\mu_{0} y\right)+\cosh \left(\mu_{0} y\right)}{\sinh \mu_{0}} \frac{1}{4}\left(\zeta(\varepsilon x)-\overline{\zeta(\varepsilon x)}+k \varepsilon^{2} R_{1},\right. \\
& \check{F}_{3}^{(2)}=-\mathrm{i} \varepsilon^{2} \zeta_{\delta}^{\star}(\varepsilon x) \frac{\mu_{0}\left(\sinh \left(\mu_{0} y\right)-\mu_{0} y \cosh \left(\mu_{0} y\right)\right)}{\sinh \left(\mu_{0}\right)}\left(\frac{1}{2} \zeta(\varepsilon x) e^{2 \mathrm{i} \mu_{0} x}-\frac{1}{2} \overline{\zeta(\varepsilon x)} e^{-2 \mathrm{i} \mu_{0} x}\right)+\varepsilon R_{1} .
\end{aligned}
$$

Similarly, the next proposition is obtained from equation (51) using Lemma 3.13 (i) with $\underline{\mu}= \pm 2 \mu_{0}$ (and $\left.q_{\min }=2 \mu_{0}-2 \delta, q_{\max }=\left(\left(2 \mu_{0}+2 \delta\right)^{2}+\lambda^{\star 2}\right)^{1 / 2}\right)$, Lemma 3.13)(ii) (with $\left.\bar{q}_{\max }=\left(4 \delta^{2}+\lambda^{\star 2}\right)^{1 / 2}\right)$ and Proposition 3.1 .

Proposition 3.16 The function $\tilde{\Gamma}^{(2)}\left(\eta_{1}\right)$ is given by the formula

$$
\begin{aligned}
\tilde{\Gamma}^{(2)}\left(\eta_{1}\right)=- & 2 \mathrm{i} \varepsilon^{2} \zeta_{\delta}^{\star}(\varepsilon x)\left(\frac{1}{2} \zeta(\varepsilon x) e^{2 \mathrm{i} \mu_{0} x}-\frac{1}{2} \overline{\zeta(\varepsilon x)} e^{-2 \mathrm{i} \mu_{0} x}\right) W(y) \\
+ & \varepsilon \frac{\mu_{0} \cosh \mu_{0}}{\sinh \mu_{0}} \mathcal{F}^{-1}\left[\frac{\mathrm{i} \mu}{\mu^{2}+k^{2}} \mathcal{F}\left[\operatorname{Re}\left(\zeta^{\star} \zeta\right)\right](\mu)\right](\varepsilon x)+\varepsilon S,
\end{aligned}
$$

where

$$
W(y)=-\frac{\mu_{0} \cosh \left(2 \mu_{0} y\right)}{4 \sinh ^{2}\left(\mu_{0}\right)}+\frac{\mu_{0} y \sinh \left(\mu_{0} y\right)}{2 \sinh \left(\mu_{0}\right)}
$$

and the symbol $S$ denotes a quantity which satisfies the estimate

$$
\|\nabla S\|_{1}+k \varepsilon\|S\|_{0} \leq c \varepsilon^{3 / 2}\|\zeta\|_{1} .
$$

Finally, we combine equation 49 with the representations of $\check{\Gamma}^{(1)}$ and $\tilde{\Gamma}^{(2)}\left(\eta_{1}\right)$ in Propositions 3.14 and 3.16 , 
Lemma 3.17 The quantity $\check{\mathcal{N}}^{(2)}\left(\eta_{1}\right)$ is given by the formula

$$
\begin{gathered}
\check{\mathcal{N}}^{(2)}\left(\eta_{1}\right)=-\varepsilon^{2} g_{0}\left(2 \mu_{0}, 0\right) B_{1} \zeta_{\delta}^{\star}(\varepsilon x)\left(\frac{1}{2} \zeta(\varepsilon x) e^{2 \mathrm{i} \mu_{0} x}+\frac{1}{2} \overline{\zeta(\varepsilon x)} e^{-2 \mathrm{i} \mu_{0} x}\right) \\
-\varepsilon^{2} \frac{\mu_{0} \cosh \left(\mu_{0}\right)}{\sinh \left(\mu_{0}\right)} \mathcal{F}^{-1}\left[\frac{\mu^{2}}{\mu^{2}+k^{2}} \mathcal{F}\left[\operatorname{Re}\left(\zeta_{\delta}^{\star} \zeta\right)\right]\right](\varepsilon x) \\
-\varepsilon^{2} \frac{\mu_{0}^{2}}{2 \sinh ^{2}\left(\mu_{0}\right)} \operatorname{Re}\left(\zeta_{\delta}^{\star}(\varepsilon x) \zeta(\varepsilon x)\right)+\varepsilon R_{0},
\end{gathered}
$$

where

$$
B_{1}=\frac{\mu_{0}^{2}\left(\cosh \left(2 \mu_{0}\right)+2\right)}{2 g_{0}\left(2 \mu_{0}, 0\right) \sinh ^{2} \mu_{0}} .
$$

We now compute the leading-order terms in the quantity

$$
\begin{aligned}
& \check{\mathcal{N}}^{(3)}\left(\eta_{1}\right)=\check{\Gamma}_{x}^{(3)}\left(\eta_{1}\right)+\left.\tilde{\Gamma}_{x}^{(2)}\left(\check{\eta}_{2}^{(2)}\right)\right|_{y=1}-\frac{3 \beta_{0}}{2}\left[\left(\eta_{1 x}^{\star}\right)^{2} \eta_{1 x}\right]_{x}+\frac{1}{2} k^{2} \varepsilon^{2} \beta_{0}\left(\eta_{1 x}^{\star}\right)^{2} \eta_{1} \\
&-\quad \mathrm{i} k \varepsilon \int_{0}^{1}\left\{-\mathrm{i} k \varepsilon y^{2}\left(\Phi_{1 y}^{\star}\right)^{2} \eta_{1}+\mathrm{i} k \varepsilon y \Phi_{1 y}^{\star} \check{\Gamma}^{(2)}-\mathrm{i} k \varepsilon y \Phi_{2 y}^{\star} \check{\Gamma}^{(1)}\right\} \mathrm{d} y \\
&- \int_{0}^{1}\left\{\Phi_{1 x}^{\star} \check{\Gamma}_{x}^{(2)}-\Phi_{1 y}^{\star} \check{\Gamma}_{y}^{(2)}+2 \Phi_{1 y}^{\star} \eta_{1}^{\star} \check{\Gamma}_{y}^{(1)}+\left(\Phi_{1 y}^{\star}\right)^{2} \eta_{1}\right\} \mathrm{d} y \\
&-\int_{0}^{1}\left[y \Phi_{1 y}^{\star} \check{\Gamma}_{x}^{(2)}+y \Phi_{1 x}^{\star} \check{\Gamma}_{y}^{(2)}-2 y^{2} \eta_{1 x}^{\star} \Phi_{1 y}^{\star} \check{\Gamma}_{y}^{(1)}-y^{2}\left(\Phi_{1 y}^{\star}\right)^{2} \eta_{1 x}\right]_{x} \mathrm{~d} y \\
&-\int_{0}^{1}\left\{\Phi_{2 x}^{\star} \check{\Gamma}_{x}^{(1)}\left(\eta_{1}\right)-\Phi_{2 y}^{\star} \check{\Gamma}_{y}^{(1)}+\left[y \Phi_{2 y}^{\star} \check{\Gamma}_{x}^{(1)}+y \Phi_{2 x}^{\star} \check{\Gamma}_{y}^{(1)}\right]_{x}\right\} \mathrm{d} y,
\end{aligned}
$$

which formula follows from equations $(46),(47)$ and the relations

$$
\check{\mathcal{N}}^{(3)}\left(\eta_{1}\right)=\tilde{\mathcal{N}}^{(3)}\left(\eta_{1}\right)+\tilde{\mathcal{N}}^{(2)}\left(\check{\eta}_{2}^{(2)}\left(\eta_{1}\right)\right), \quad \check{\Gamma}^{(2)}=\tilde{\Gamma}^{(2)}\left(\eta_{1}\right)+\tilde{\Gamma}^{(1)}\left(\check{\eta}_{2}^{(2)}\right)
$$

For this purpose we also use the formulae

$$
\tilde{\Gamma}^{(1)}\left(\check{\eta}_{2}^{(2)}\right)=\mathcal{F}^{-1}\left[-\mathrm{i} \mu G(y, 1) \mathcal{F}\left[\check{\eta}_{2}^{(2)}\right]\right], \quad \check{\eta}_{2}^{(2)}=g_{\varepsilon}(D, \lambda)^{-1}(1-\chi(D)) \tilde{\mathcal{N}}^{(2)}\left(\eta_{1}\right),
$$

and

$$
\tilde{\Gamma}^{(3)}\left(\eta_{1}\right)+\tilde{\Gamma}^{(2)}\left(\check{\eta}_{2}^{(2)}\right)=\mathcal{F}^{-1}\left[\int_{0}^{1}\left(G(y, \tilde{y})\left(-\mathrm{i} \mu \mathcal{F}\left[\check{F}_{1}^{(3)}\right]-\mathrm{i} \lambda \mathcal{F}\left[\check{F}_{2}^{(3)}\right]\right)+G_{\tilde{y}}(y, \tilde{y}) \mathcal{F}\left[\check{F}_{3}^{(3)}\right]\right) \mathrm{d} \tilde{y}\right],
$$

where

$$
\begin{aligned}
\check{F}_{1}^{(3)}=- & \eta_{1}^{\star} \check{\Gamma}_{x}^{(2)}-\eta_{2}^{\star} \check{\Phi}_{x}^{(1)}-\Phi_{1 x}^{\star} \check{\eta}_{2}^{(2)}-\Phi_{2 x}^{\star} \eta_{1}+y \Phi_{1 y}^{\star} \check{\eta}_{2 x}^{(2)}+y \Phi_{2 y}^{\star} \eta_{1 x} \\
& \quad+y \eta_{1 x}^{\star} \check{\Phi}_{y}^{(2)}+y \eta_{2 x}^{\star} \check{\Gamma}_{y}^{(1)}, \\
\check{F}_{2}^{(3)}=-\mathrm{i} k \varepsilon \varepsilon \eta_{1}^{\star} \check{\Gamma}^{(2)}-\mathrm{i} k \varepsilon \eta_{2}^{\star} \check{\Gamma}^{(1)}+\mathrm{i} k \varepsilon y \Phi_{2 y}^{\star} \eta_{1}+\mathrm{i} k \varepsilon y \Phi_{1 y}^{\star} \check{\eta}_{2}^{(2)}, & \check{F}_{3}^{(3)}=y \\
\quad & \eta_{1 x}^{\star} \check{\Gamma}_{x}^{(2)}+y \eta_{2 x}^{\star} \check{\Gamma}_{x}^{(1)}+y \Phi_{1 x}^{\star} \check{\eta}_{2 x}^{(2)}+y \Phi_{2 x}^{\star} \eta_{1 x}+\eta_{1}^{\star} \check{\Gamma}_{y}^{(2)}+\eta_{2}^{\star} \check{\Gamma}_{y}^{(1)} \\
& \quad+\Phi_{1 y}^{\star} \check{\eta}_{2}^{(2)}+\Phi_{2 y}^{\star} \eta_{1}-\left(\eta_{1}^{\star}\right)^{2} \check{\Gamma}_{y}^{(1)}-2 \Phi_{1 y}^{\star} \eta_{1}^{\star} \eta_{1}-y^{2}\left(\eta_{1 x}^{\star}\right)^{2} \check{\Gamma}_{y}^{(1)}-2 y^{2} \eta_{1 x}^{\star} \Phi_{1 y}^{\star} \eta_{1 x} .
\end{aligned}
$$


Proposition 3.18 The quantity $\check{\eta}_{2}^{(2)}$ satisfies the estimate

$$
\begin{aligned}
\check{\eta}_{2}^{(2)}= & -\varepsilon^{2} B_{1} \zeta_{\delta}^{\star}(\varepsilon x)\left(\frac{1}{2} \zeta(\varepsilon x) e^{2 \mathrm{i} \mu_{0} x}+\frac{1}{2} \overline{\zeta(\varepsilon x)} e^{-2 \mathrm{i} \mu_{0} x}\right)-\varepsilon^{2} B_{2} \operatorname{Re}\left(\zeta_{\delta}^{\star}(\varepsilon x) \zeta(\varepsilon x)\right) \\
& -\varepsilon^{2} B_{3} \mathcal{F}^{-1}\left[\frac{\mu^{2}}{\left(1-\alpha_{0}^{-1}\right) \mu^{2}+k^{2}} \mathcal{F}\left[2 \operatorname{Re}\left(\zeta_{\delta}^{\star} \zeta\right)\right](\mu)\right](\varepsilon x)+\varepsilon R_{2},
\end{aligned}
$$

where

$$
B_{2}=\frac{\mu_{0}^{2}}{2 \alpha_{0} \sinh ^{2} \mu_{0}}, \quad B_{3}=\alpha_{0}^{-1} A_{4}
$$

Proof. We apply the operator $g_{\varepsilon}(D, \lambda)^{-1}(1-\chi(D))$ to the expression for $\check{\mathcal{N}}^{(2)}\left(\eta_{1}\right)$ given in Lemma 3.17. The first term on the right-hand side of this expression is handled by the approximation

$$
g_{\varepsilon}(\mu, k \varepsilon)^{-1}=g_{0}\left(2 \mu_{0}, 0\right)^{-1}+T_{1}(\mu, \varepsilon)
$$

where

$$
\left|T_{1}(\mu, \varepsilon)\right| \leq c\left(\left|\mu-2 \mu_{0}\right|+\varepsilon^{2}\right), \quad\left|\mu-2 \mu_{0}\right| \leq 2 \delta,
$$

while the second and third are treated with the approximation

$$
g_{\varepsilon}(\mu, k \varepsilon)^{-1}=\frac{q^{2}}{\left(\alpha_{0}-1\right) \mu^{2}+\alpha_{0} k^{2} \varepsilon^{2}}+T_{2}(\mu, \varepsilon)
$$

where

$$
\left|T_{2}(q, \varepsilon)\right| \leq c\left(\mu^{2}+\varepsilon^{2}\right), \quad|\mu|<2 \delta .
$$

Turning to the fourth term, note that

$$
g_{\varepsilon}(D, \lambda)^{-1}(1-\chi(D)) R_{0}=R_{2}
$$

because $g_{\varepsilon}(\mu, k \varepsilon)>c\left(1+q^{2}\right)$ for $\mu \in \operatorname{supp}(1-\chi)$ (see Proposition 3.3.

The function $\tilde{\Gamma}^{(1)}\left(\check{\eta}_{2}^{(2)}\right)$ may now be computed using Lemma 3.13, combining the result with Proposition 3.16 yields the following proposition.

Proposition 3.19 The function $\check{\Gamma}^{(2)}$ is given by the formula

$$
\check{\Gamma}^{(2)}=-\mathrm{i} \varepsilon^{2} \zeta_{\delta}^{\star}(\varepsilon x)\left(\frac{1}{2} \zeta(\varepsilon x) e^{2 \mathrm{i} \mu_{0} x}-\frac{1}{2} \overline{\zeta(\varepsilon x)} e^{-2 \mathrm{i} \mu_{0} x}\right) M_{1}(y)+\varepsilon \vartheta_{\delta}(\varepsilon x)+\varepsilon S,
$$

where

$$
\begin{aligned}
\vartheta_{\delta}(x) & =A_{4} \mathcal{F}^{-1}\left[\frac{\mathrm{i} \mu}{\left(1-\alpha_{0}^{-1}\right) \mu^{2}+k^{2}} \mathcal{F}\left[2 \operatorname{Re}\left(\zeta_{\delta}^{\star} \zeta\right)\right]\right](x), \\
M_{1}(y) & =M_{11} \frac{\cosh \left(2 \mu_{0} y\right)}{\sinh \left(2 \mu_{0}\right)}+\frac{\mu_{0} y \sinh \left(\mu_{0} y\right)}{\sinh \left(\mu_{0}\right)}, \\
M_{11} & =-\frac{\mu_{0}^{2}\left(\cosh \left(2 \mu_{0}\right)+2\right)+\mu_{0} g_{0}\left(2 \mu_{0}, 0\right) \sinh \left(2 \mu_{0}\right)}{2 \sinh ^{2}\left(\mu_{0}\right) g_{0}\left(2 \mu_{0}, 0\right)} .
\end{aligned}
$$


Corollary 3.20 The functions $\check{F}_{1}^{(3)}, \check{F}_{2}^{(3)}$ and $\check{F}_{3}^{(3)}$ are given by the formulae

$$
\begin{aligned}
& \check{F}_{1}^{(3)}= \varepsilon^{3}\left(\zeta_{\delta}^{\star}(\varepsilon x)\right)^{2}\left(\frac{1}{2}\left(\zeta(\varepsilon x) U_{11}(y)+\overline{\zeta(\varepsilon x)} U_{12}(y)\right) \mathrm{e}^{\mathrm{i} \mu_{0} x}+\frac{1}{2}\left(\zeta(\varepsilon x) U_{12}(y)+\overline{\zeta(\varepsilon x)} U_{11}(y)\right) \mathrm{e}^{-\mathrm{i} \mu_{0} x}\right) \\
&+\varepsilon^{3} \zeta_{\delta}^{\star}(\varepsilon x) \vartheta_{\delta x}(\varepsilon x) U_{2}(y)\left(\frac{1}{2} \mathrm{e}^{\mathrm{i} \mu_{0} x}+\frac{1}{2} \mathrm{e}^{-\mathrm{i} \mu_{0} x}\right)+\varepsilon^{2} T, \\
& \check{F}_{2}^{(3)}=\varepsilon T, \\
& \check{F}_{3}^{(3)}=-\mathrm{i} \varepsilon^{3}\left(\zeta_{\delta}^{\star}(\varepsilon x)\right)^{2}\left(\frac{1}{2}\left(\zeta(\varepsilon x) U_{31}(y)+\overline{\zeta(\varepsilon x)} U_{32}(y)\right) \mathrm{e}^{\mathrm{i} \mu_{0} x}-\frac{1}{2}\left(\zeta(\varepsilon x) U_{32}(y)+\overline{\zeta(\varepsilon x)} U_{31}(y)\right) \mathrm{e}^{-\mathrm{i} \mu_{0} x}\right) \\
& \quad-\mathrm{i} \varepsilon^{3} \zeta_{\delta}^{\star}(\varepsilon x) \vartheta_{\delta x}(\varepsilon x) U_{4}(y)\left(\frac{1}{2} \mathrm{e}^{\mathrm{i} \mu_{0} x}-\frac{1}{2} \mathrm{e}^{-\mathrm{i} \mu_{0} x}\right)+\varepsilon^{2} T,
\end{aligned}
$$

where

$$
\begin{aligned}
& U_{11}(y)=\frac{\mu_{0}\left(\cosh \left(\mu_{0} y\right)+2 \mu_{0} y \sinh \left(\mu_{0} y\right)\right) B_{1}}{2 \sinh \left(\mu_{0}\right)}+\frac{\mu_{0} \cosh \left(\mu_{0} y\right)\left(C_{2}+\frac{1}{2} B_{2}\right)}{\sinh \left(\mu_{0}\right)} \\
& -C_{4}-\frac{\mu_{0} y M_{1}^{\prime}(y)}{2}-\mu_{0} M_{1}(y) \\
& U_{12}(y)=\frac{\mu_{0}\left(\cosh \left(\mu_{0} y\right)+2 \mu_{0} y \sinh \left(\mu_{0} y\right)\right) C_{1}}{2 \sinh \left(\mu_{0}\right)}+\frac{\mu_{0} \cosh \left(\mu_{0} y\right) B_{2}}{2 \sinh \left(\mu_{0}\right)} \\
& -\frac{\mu_{0} C_{5}\left(\cosh \left(2 \mu_{0} y\right)+\mu_{0} y \sinh \left(2 \mu_{0} y\right)\right)}{\sinh \left(2 \mu_{0}\right)}-\frac{\mu_{0}^{2} y\left(y \mu_{0} \cosh \left(\mu_{0} y\right)+3 \sinh \left(\mu_{0} y\right)\right)}{4 \sinh \left(\mu_{0}\right)}, \\
& U_{2}(y)=-1-\frac{\mu_{0} \cosh \left(\mu_{0} y\right)}{\alpha_{0} \sinh \left(\mu_{0}\right)}, \\
& U_{31}(y)=\frac{\mu_{0}\left(\sinh \left(\mu_{0} y\right)+2 \mu_{0} y \cosh \left(\mu_{0} y\right)\right) B_{1}}{2 \sinh \left(\mu_{0}\right)}-\frac{\mu_{0} \sinh \left(\mu_{0} y\right)\left(C_{2}+\frac{1}{2} B_{2}\right)}{\sinh \left(\mu_{0}\right)} \\
& -C_{4} \mu_{0} y+\frac{M_{1}^{\prime}(y)}{2}+\mu_{0}^{2} y M_{1}(y)-\frac{\mu_{0} \sinh \left(\mu_{0} y\right)\left(1+3 \mu_{0}^{2} y^{2}\right)}{2 \sinh \left(\mu_{0}\right)}, \\
& U_{32}(y)=\frac{\mu_{0}\left(\sinh \left(\mu_{0} y\right)+2 \mu_{0} y \cosh \left(\mu_{0} y\right)\right) C_{1}}{2 \sinh \left(\mu_{0}\right)}-\frac{\mu_{0} \sinh \left(\mu_{0} y\right) B_{2}}{2 \sinh \left(\mu_{0}\right)} \\
& +\frac{\mu_{0} C_{5}\left(\mu_{0} y \cosh \left(2 \mu_{0} y\right)+\sinh \left(2 \mu_{0} y\right)\right)}{\sinh \left(2 \mu_{0}\right)}+\frac{y \mu_{0}^{2}\left(\cosh \left(\mu_{0} y\right)-\mu_{0} y \sinh \left(\mu_{0} y\right)\right.}{4 \sinh \left(\mu_{0}\right)} \\
& U_{4}(y)=-\mu_{0} y+\frac{\mu_{0} \sinh \left(\mu_{0} y\right)}{\alpha_{0} \sinh \left(\mu_{0}\right)}
\end{aligned}
$$

and the symbol $T$ denotes a quantity which satisfies the estimate

$$
\|\chi(D) T\|_{0} \leq c \varepsilon^{3 / 2}\|\zeta\|_{1} .
$$

The next proposition is obtained from equation (56) using Lemma 3.13 and Proposition 3.1. 
Proposition 3.21 The quantity $\tilde{\Gamma}_{x}^{(3)}\left(\eta_{1}\right)+\left.\tilde{\Gamma}_{x}^{(2)}\left(\check{\eta}_{2}^{(2)}\right)\right|_{y=1}$ is given by the formula

$$
\begin{aligned}
\tilde{\Gamma}_{x}^{(3)}\left(\eta_{1}\right)+ & \left.\tilde{\Gamma}_{x}^{(2)}\left(\check{\eta}_{2}^{(2)}\right)\right|_{y=1} \\
= & \mu_{0} \varepsilon^{2}\left(\zeta_{\delta}(\varepsilon x)\right)^{2}\left(\frac{1}{2}\left(\zeta(\varepsilon x) N_{1}+\overline{\zeta(\varepsilon x)} N_{2}\right) e^{\mathrm{i} \mu_{0} x}+\frac{1}{2}\left(\zeta(\varepsilon x) N_{2}+\overline{\zeta(\varepsilon x)} N_{1}\right) e^{-\mathrm{i} \mu_{0} x}\right) \\
& \quad+\mu_{0} \varepsilon^{3} \zeta_{\delta}^{\star}(\varepsilon x) \vartheta_{\delta x}(\varepsilon x) N_{3}\left(\frac{1}{2} \mathrm{e}^{\mathrm{i} \mu_{0} x}+\frac{1}{2} \mathrm{e}^{-\mathrm{i} \mu_{0} x}\right)+\varepsilon^{2} T,
\end{aligned}
$$

where

$$
\begin{aligned}
& N_{1}=\frac{\mu_{0} \cosh \left(2 \mu_{0}\right) B_{1}}{2 \sinh ^{2}\left(\mu_{0}\right)}+\frac{\mu_{0}\left(C_{2}+\frac{1}{2} B_{2}\right)}{\sinh ^{2}\left(\mu_{0}\right)}-\frac{\cosh \left(\mu_{0}\right) C_{4}}{\sinh \left(\mu_{0}\right)}-\frac{M_{11} \mu_{0}}{2 \sinh ^{2}\left(\mu_{0}\right)}-\frac{\mu_{0}^{2} \cosh \left(\mu_{0}\right)}{2 \sinh \left(\mu_{0}\right)}, \\
& N_{2}=\frac{\mu_{0} \cosh \left(2 \mu_{0}\right) C_{1}}{2 \sinh ^{2}\left(\mu_{0}\right)}+\frac{\mu_{0} B_{2}}{2 \sinh ^{2}\left(\mu_{0}\right)}-\frac{\mu_{0} C_{5}}{2 \sinh ^{2}\left(\mu_{0}\right)}-\frac{\mu_{0}^{2} \cosh \left(\mu_{0}\right)}{4 \sinh \left(\mu_{0}\right)}, \\
& N_{3}=\frac{-2 \mu_{0} \alpha_{0}^{-1}-\sinh \left(2 \mu_{0}\right)}{2 \sinh ^{2}\left(\mu_{0}\right)} .
\end{aligned}
$$

Finally, we combine equation (55) with Propositions 3.19 and 3.21 .

Lemma 3.22 The quantity $\check{\mathcal{N}}^{(3)}\left(\eta_{1}\right)$ is given by the formula

$$
\begin{aligned}
\check{\mathcal{N}}^{(3)}\left(\eta_{1}\right)=\varepsilon^{3} & \left(\zeta_{\delta}^{\star}(\varepsilon x)\right)^{2}\left(\left(A_{3}+A_{5}\right) \zeta(\varepsilon x)+A_{3} \overline{\zeta(\varepsilon x)}\right) \frac{\mathrm{e}^{\mathrm{i} \mu_{0} x}}{2} \\
& +\varepsilon^{3}\left(\zeta_{\delta}^{\star}(\varepsilon x)\right)^{2}\left(A_{3} \zeta(\varepsilon x)+\left(A_{3}+A_{5}\right) \overline{\zeta(\varepsilon x)}\right) \frac{\mathrm{e}^{-\mathrm{i} \mu_{0} x}}{2} \\
& -4 \varepsilon^{3} A_{4} \zeta_{\delta}^{\star}(\varepsilon x) \vartheta_{\delta x}(\varepsilon x)\left(\frac{1}{2} \mathrm{e}^{\mathrm{i} \mu_{0} x}+\frac{1}{2} \mathrm{e}^{-\mathrm{i} \mu_{0} x}\right)+\varepsilon^{2} T .
\end{aligned}
$$

\subsection{Derivation of the reduced equation in its final form}

In this section we derive the reduced equation in its final form, thus completing the proof of Theorem 2.2(ii) and (iii). For $k>0$ we define $\check{u}_{\varepsilon, k}: \chi_{0}(\varepsilon D) L^{2}(\mathbb{R}) \times X^{0} \rightarrow Y^{1}$ by the formula

$$
\check{u}_{\varepsilon, k}\left(\zeta, u^{\dagger}\right)=\left(\eta_{1}+\check{\eta}_{2}\left(\eta_{1}, u^{\dagger}\right), \check{\omega}\left(\eta_{1}, u^{\dagger}\right), \check{\Gamma}\left(\eta_{1}, u^{\dagger}\right), \check{\xi}\left(\eta_{1}, u^{\dagger}\right)\right),
$$

while $\check{u}_{\varepsilon, 0}: \chi_{0}(\varepsilon D) L_{\mathrm{c}}^{2}(\mathbb{R}) \times Z_{\mathrm{r}}^{0} \rightarrow Y_{\star}^{1}$ is defined by

$$
\check{u}_{\varepsilon, 0}\left(\zeta, \eta^{\dagger}, \omega^{\dagger}, \Gamma^{\dagger}, \xi_{1}^{\dagger}, \xi_{3}^{\dagger}\right)=\left(\eta_{1}+\check{\eta}_{2}\left(\eta_{1}, u^{\dagger}\right), \check{\omega}\left(\eta_{1}, u^{\dagger}\right), \check{\Gamma}\left(\eta_{1}, u^{\dagger}\right), \check{\xi}\left(\eta_{1}, u^{\dagger}\right)\right)
$$

with $\xi^{\dagger}=-\left(\xi_{1}^{\dagger}\right)_{x}-\left(\xi_{3}^{\dagger}\right)_{y}$; here

$$
\eta_{1}=\varepsilon \zeta(\varepsilon x) \frac{\mathrm{e}^{\mathrm{i} \mu_{0} x}}{2}+\varepsilon \overline{\zeta(\varepsilon x)} \frac{\mathrm{e}^{-\mathrm{i} \mu_{0} x}}{2}
$$

and $\check{\omega}, \check{\xi}$ are given by equations 31 , 32, with $\Phi=\check{\Gamma}\left(\eta_{1}, u^{\dagger}\right), \eta=\eta_{1}+\check{\eta}_{2}\left(\eta_{1}, u^{\dagger}\right)$. The theory in Sections $3.1-3.4$ above shows that

$$
\eta_{1}^{+}(x)=\varepsilon \zeta(\varepsilon x) \frac{\mathrm{e}^{\mathrm{i} \mu_{0} x}}{2}, \quad \zeta \in \begin{cases}\chi_{0}(\varepsilon D) L^{2}(\mathbb{R}), & k>0, \\ \chi_{0}(\varepsilon D) L_{\mathrm{c}}^{2}(\mathbb{R}), & k=0\end{cases}
$$


solves the reduced equation

$$
g_{\varepsilon}(\mu, k \varepsilon) \hat{\eta}_{1}^{+}=\chi_{+} \mathcal{F}\left[\check{\mathcal{N}}^{(3)}\left(\eta_{1}\right)+\check{\mathcal{N}}^{\mathrm{r}}\left(\eta_{1}\right)+\check{\mathcal{N}}^{\dagger}\left(u^{\dagger}\right)\right]
$$

where $\eta_{1}=\eta_{1}^{+}+\overline{\eta_{1}^{+}}$, if and only if $u=\check{u}_{\varepsilon, k}$ solves the resolvent equations $\sqrt{25}-(30)$. It follows that

$$
\eta_{1}^{+}(x)=\varepsilon \zeta(\varepsilon x) \frac{\mathrm{e}^{\mathrm{i} \mu_{0} x}}{2}, \quad \zeta \in \begin{cases}L^{2}(\mathbb{R}), & k>0, \\ L_{\mathrm{c}}^{2}(\mathbb{R}), & k=0,\end{cases}
$$

solves the equation

$$
g_{\varepsilon}(\mu, k \varepsilon) \hat{\eta}_{1}^{+}=\chi_{+} \mathcal{F}\left[\check{\mathcal{N}}^{(3)}\left(\chi(D) \eta_{1}\right)+\check{\mathcal{N}}^{\mathrm{r}}\left(\chi(D) \eta_{1}\right)+\check{\check{\mathcal{N}}}^{\dagger}\left(u^{\dagger}\right)\right],
$$

where $\eta_{1}=\eta_{1}^{+}+\overline{\eta_{1}^{+}}$, if and only if $\zeta \in \chi_{0}(\varepsilon D) L^{2}(\mathbb{R})$ (for $k>0$ ) or $\zeta \in \chi_{0}(\varepsilon D) L_{\mathrm{c}}^{2}(\mathbb{R})$ (for $k=0)$ and $u=\check{u}_{\varepsilon, k}$ solves the resolvent equations $(25)-(30)$.

Since all solutions of $(57)$ have support in the interval $\left[\mu_{0}-\delta, \mu_{0}+\delta\right]$, its solution set in $L^{2}(\mathbb{R})$ coincides with its solution set in $H^{s}(\mathbb{R})$ for any $s \geq 0$, and we henceforth work in $H^{2}(\mathbb{R})$ by writing it as a second-order pseudodifferential equation. Let

$$
\tilde{g}_{\varepsilon}(\mu, \lambda)=\varepsilon^{2}+A_{1}\left(\mu-\mu_{0}\right)^{2}+A_{2} \lambda^{2}
$$

be the second-order Taylor polynomial of $g_{\varepsilon}$ at the point $\left(\mu_{0}, 0\right)$ and write $(57)$ as

$$
\tilde{g}_{\varepsilon}(\mu, \varepsilon k) \hat{\eta}_{1}^{+}=\frac{\tilde{g}_{\varepsilon}(\mu, \varepsilon k)}{g_{\varepsilon}(\mu, \varepsilon k)} \chi_{+} \mathcal{F}\left[\check{\mathcal{N}}^{(3)}\left(\chi(D) \eta_{1}\right)+\check{\mathcal{N}}^{\mathrm{r}}\left(\chi(D) \eta_{1}\right)+\check{\mathcal{N}}^{\dagger}\left(u^{\dagger}\right)\right]
$$

or equivalently as

$\tilde{g}_{\varepsilon}\left(\mu_{0}+\varepsilon \tilde{\mu}, \varepsilon k\right) \hat{\zeta}(\tilde{\mu})=2 \frac{\tilde{g}_{\varepsilon}\left(\mu_{0}+\varepsilon \tilde{\mu}, \varepsilon k\right)}{g_{\varepsilon}\left(\mu_{0}+\varepsilon \tilde{\mu}, \varepsilon k\right)} \chi_{0}(\varepsilon \tilde{\mu}) \mathcal{F}\left[\check{\mathcal{N}}^{(3)}\left(\chi(D) \eta_{1}\right)+\check{\mathcal{N}}^{\mathrm{r}}\left(\chi(D) \eta_{1}\right)+\check{\mathcal{N}}^{\dagger}\left(u^{\dagger}\right)\right]\left(\mu_{0}+\varepsilon \tilde{\mu}\right)$.

Taking the inverse Fourier transform with respect to $\tilde{\mu}$, we find that

$$
\begin{aligned}
& A_{2}^{-1} \zeta-A_{2}^{-1} A_{1} \zeta_{x x}+k^{2} \zeta \\
& =\mathcal{F}^{-1}\left[2 \frac{\tilde{g}_{\varepsilon}\left(\mu_{0}+\varepsilon \tilde{\mu}, \varepsilon k\right)}{g_{\varepsilon}\left(\mu_{0}+\varepsilon \tilde{\mu}, \varepsilon k\right)} \chi_{0}(\varepsilon \tilde{\mu}) \varepsilon^{-2} A_{2}^{-1}\right. \\
& \left.\quad \times \mathcal{F}\left[\check{\mathcal{N}}^{(3)}\left(\chi(D) \eta_{1}\right)+\check{\mathcal{N}}^{\mathrm{r}}\left(\chi(D) \eta_{1}\right)\right]\left(\mu_{0}+\varepsilon \tilde{\mu}\right)+\hat{\zeta}_{\varepsilon, k}^{\dagger}\left(u^{\dagger}\right)(\tilde{\mu})\right],
\end{aligned}
$$

where

$$
\mathcal{F}\left[\zeta_{\varepsilon, k}^{\dagger}\left(u^{\dagger}\right)\right](\tilde{\mu})=2 A_{2}^{-1} \frac{\tilde{g}_{\varepsilon}\left(\mu_{0}+\varepsilon \tilde{\mu}, \varepsilon k\right)}{g_{\varepsilon}\left(\mu_{0}+\varepsilon \tilde{\mu}, \varepsilon k\right)} \chi_{0}(\varepsilon \tilde{\mu}) \varepsilon^{-2} \mathcal{F}\left[\check{\mathcal{N}}^{\dagger}\left(u^{\dagger}\right)\right]\left(\mu_{0}+\varepsilon \tilde{\mu}\right) .
$$

Observe that

$$
\begin{aligned}
& \check{\mathcal{N}}^{(3)}\left(\chi(D) \eta_{1}\right) \\
& =\varepsilon^{3}\left(\zeta_{\delta}^{\star}(\varepsilon x)\right)^{2}\left(\left(A_{3}+A_{5}\right)\left(\chi_{0}(\varepsilon D) \zeta\right)(\varepsilon x)+A_{3} \overline{\left(\chi_{0}(\varepsilon D) \zeta\right)(\varepsilon x)}\right) \frac{\mathrm{e}^{\mathrm{i} \mu_{0} x}}{2} \\
& \quad+\varepsilon^{3}\left(\zeta_{\delta}^{\star}(\varepsilon x)\right)^{2}\left(A_{3}\left(\chi_{0}(\varepsilon D) \zeta\right)(\varepsilon x)+\left(A_{3}+A_{5} \overline{\left(\chi_{0}(\varepsilon D) \zeta\right)(\varepsilon x)}\right) \frac{\mathrm{e}^{-\mathrm{i} \mu_{0} x}}{2}\right. \\
& \quad+\varepsilon^{3} A_{4} \zeta_{\delta}^{\star}(\varepsilon x)\left(\chi_{0}(\varepsilon D) \vartheta_{\delta}\right)_{x}(\varepsilon x)\left(\frac{1}{2} \mathrm{e}^{\mathrm{i} \mu_{0} x}+\frac{1}{2} \mathrm{e}^{-\mathrm{i} \mu_{0} x}\right)+\varepsilon^{2} T,
\end{aligned}
$$


and using the estimate

$$
\left\|\left(\chi_{0}(\varepsilon \tilde{\mu})-1\right) \hat{f}(\tilde{\mu})\right\|_{0}^{2}=\int_{|\tilde{\mu}| \geq \delta / \varepsilon}|\hat{f}(\tilde{\mu})|^{2} \mathrm{~d} \tilde{\mu} \leq \delta^{-2} \varepsilon^{2} \int_{|\tilde{\mu}| \geq \delta / \varepsilon}|\tilde{\mu}|^{2}|\hat{f}(\tilde{\mu})|^{2} \mathrm{~d} \tilde{\mu} \leq \delta^{-2} \varepsilon^{2}\|f\|_{1},
$$

we find that

$$
\left\|\left(\left(\chi_{0}(\varepsilon D)-1\right) \zeta\right)(\varepsilon x) \mathrm{e}^{\mathrm{i} \mu_{0} x}\right\|_{0}^{2}=\varepsilon^{-1}\left\|\left(\chi_{0}(\varepsilon \tilde{\mu})-1\right) \hat{\zeta}(\tilde{\mu})\right\|_{0}^{2} \leq \delta^{-2} \varepsilon\|\zeta\|_{1}
$$

and

$$
\begin{aligned}
\left\|\left(\left(\chi_{0}(\varepsilon D)-1\right) \vartheta_{\delta}\right)_{x}(\varepsilon x) \mathrm{e}^{\mathrm{i} \mu_{0} x}\right\|_{0}^{2} & =\varepsilon^{-1} A_{4}\left\|\frac{\left(\chi_{0}(\varepsilon \tilde{\mu})-1\right) \tilde{\mu}^{2}}{\left(1-\alpha_{0}^{-1}\right) \tilde{\mu}^{2}+k^{2}} \mathcal{F}\left[2 \operatorname{Re}\left(\zeta_{\delta}^{\star} \zeta\right)\right](\tilde{\mu})\right\|_{0} \\
& \leq c \varepsilon^{-1}\left\|\left(\chi_{0}(\varepsilon \tilde{\mu})-1\right) \mathcal{F}\left[2 \operatorname{Re}\left(\zeta_{\delta}^{\star} \zeta\right)\right](\tilde{\mu})\right\|_{0} \\
& \leq c \varepsilon\|\zeta\|_{1},
\end{aligned}
$$

so that

$$
\check{\mathcal{N}}^{(3)}\left(\chi(D) \eta_{1}\right)=\frac{\varepsilon^{3}}{2} E(\varepsilon x) \mathrm{e}^{\mathrm{i} \mu_{0} x}+\frac{\varepsilon^{3}}{2} F(\varepsilon x) \mathrm{e}^{-\mathrm{i} \mu_{0} x}+\varepsilon^{2} T,
$$

where

$$
\begin{aligned}
& E=\left(A_{3}+A_{5}\right)\left(\zeta_{\delta}^{\star}\right)^{2} \zeta+A_{3}\left(\zeta_{\delta}^{\star}\right)^{2} \bar{\zeta}-4 A_{4} \zeta_{\delta}^{\star} \vartheta_{\delta x}, \\
& F=A_{3}\left(\zeta_{\delta}^{\star}\right)^{2} \chi_{0}(\varepsilon D) \zeta+\left(A_{3}+A_{5}\right)\left(\zeta_{\delta}^{\star}\right)^{2} \chi_{0}(\varepsilon D) \bar{\zeta}-4 A_{4} \zeta_{\delta}^{\star}\left(\chi_{0}(\varepsilon D) \vartheta_{\delta}\right)_{x} .
\end{aligned}
$$

Furthermore

$$
\chi_{0}(\varepsilon \tilde{\mu}) \mathcal{F}\left[\varepsilon F(\varepsilon x) \mathrm{e}^{-\mathrm{i} \mu_{0} x}\right]\left(\mu_{0}+\varepsilon \tilde{\mu}\right)=\chi_{0}(\varepsilon \tilde{\mu}) \hat{F}\left(\tilde{\mu}+2 \varepsilon^{-1} \mu_{0}\right)=0
$$

because $\operatorname{supp} \hat{F} \subseteq\left[-3 \varepsilon^{-1} \delta, 3 \varepsilon^{-1} \delta\right]$.

Using the estimate

$$
\left|\frac{\tilde{g}_{\varepsilon}(\mu, \lambda)}{g_{\varepsilon}(\mu, \lambda)}-1\right| \leq c\left|\left(\mu-\mu_{0}, \lambda\right)\right|^{2}, \quad\left|\mu-\mu_{0}\right| \leq \delta, \lambda \leq \lambda^{\star},
$$

we find in particular that

$$
\left|\frac{\tilde{g}_{\varepsilon}\left(\mu_{0}+\varepsilon \tilde{\mu}, \varepsilon k\right)}{g_{\varepsilon}\left(\mu_{0}+\varepsilon \tilde{\mu}, \varepsilon k\right)} \chi_{0}(\varepsilon \tilde{\mu})\right| \leq c .
$$

It follows that

$$
\begin{aligned}
\left\|\mathcal{F}^{-1}\left[\frac{\tilde{g}_{\varepsilon}\left(\mu_{0}+\varepsilon \tilde{\mu}, \varepsilon k\right)}{g_{\varepsilon}\left(\mu_{0}+\varepsilon \tilde{\mu}, \varepsilon k\right)} \chi_{0}(\varepsilon \tilde{\mu}) \varepsilon^{-2} \mathcal{F}\left[\check{\mathcal{N}}^{\mathrm{r}}\left(\chi(D) \eta_{1}\right)\right]\left(\mu_{0}+\varepsilon \tilde{\mu}\right)\right]\right\|_{0} & \leq c \varepsilon^{-5 / 2}\left\|\check{\mathcal{N}}^{\mathrm{r}}\left(\chi(D) \eta_{1}\right)\right\|_{0} \\
& \leq c \varepsilon^{-1 / 2}\left\|\chi(D) \eta_{1}\right\|_{0} \\
& \leq c \varepsilon\|\zeta\|_{0}
\end{aligned}
$$

and

$$
\left\|\mathcal{F}^{-1}\left[\frac{\tilde{g}_{\varepsilon}\left(\mu_{0}+\varepsilon \tilde{\mu}, \varepsilon k\right)}{g_{\varepsilon}\left(\mu_{0}+\varepsilon \tilde{\mu}, \varepsilon k\right)} \chi_{0}(\varepsilon \tilde{\mu}) \varepsilon^{-2} \mathcal{F}[T]\left(\mu_{0}+\varepsilon \tilde{\mu}\right)\right]\right\|_{0} \leq c \varepsilon^{-1 / 2}\left\|\chi_{+}(D) T\right\|_{0} \leq c \varepsilon\|\zeta\|_{1} .
$$


Finally, we write

$$
\begin{aligned}
& \mathcal{F}^{-1}\left[2 \frac{\tilde{g}_{\varepsilon}\left(\mu_{0}+\varepsilon \tilde{\mu}, \varepsilon k\right)}{g_{\varepsilon}\left(\mu_{0}+\varepsilon \tilde{\mu}, \varepsilon k\right)} \chi_{0}(\varepsilon \tilde{\mu}) \varepsilon^{-2} \mathcal{F}\left[\frac{\varepsilon^{3}}{2} E(\varepsilon x) \mathrm{e}^{\mathrm{i} \mu_{0} x}\right]\left(\mu_{0}+\varepsilon \tilde{\mu}\right)\right] \\
& =\mathcal{F}^{-1}\left[\left(\frac{\tilde{g}_{\varepsilon}\left(\mu_{0}+\varepsilon \tilde{\mu}, \varepsilon k\right)}{g_{\varepsilon}\left(\mu_{0}+\varepsilon \tilde{\mu}, \varepsilon k\right)}-1\right) \chi_{0}(\varepsilon \tilde{\mu}) \hat{E}(\tilde{\mu})\right]+\mathcal{F}^{-1}\left[\left(\chi_{0}(\varepsilon \tilde{\mu})-1\right) \hat{E}(\tilde{\mu})\right]+E(x)
\end{aligned}
$$

and note that

$$
\left\|\mathcal{F}^{-1}\left[\left(\frac{\tilde{g}_{\varepsilon}\left(\mu_{0}+\varepsilon \tilde{\mu}, \varepsilon k\right)}{g_{\varepsilon}\left(\mu_{0}+\varepsilon \tilde{\mu}, \varepsilon k\right)}-1\right) \chi_{0}(\varepsilon \tilde{\mu}) \hat{E}(\tilde{\mu})\right]\right\|_{0} \leq c\left(\varepsilon \delta\left\|E^{\prime}\right\|_{0}+k^{2} \varepsilon^{2}\|E\|_{0}\right) \leq c \varepsilon\|\zeta\|_{1},
$$

(using estimate (61)), and

$$
\left\|\mathcal{F}^{-1}\left[\left(\chi_{0}(\varepsilon \tilde{\mu})-1\right) \hat{E}(\tilde{\mu})\right]\right\|_{0} \leq c \varepsilon\|E\|_{1} \leq c \varepsilon\|\zeta\|_{1}
$$

(using estimate (59)).

According to the above calculations equation (58) can be written as

$$
\begin{aligned}
& A_{2}^{-1} \zeta-A_{1} A_{2}^{-1} \zeta_{x x}+k^{2} \zeta \\
& \quad=A_{2}^{-1}\left(A_{3}+A_{5}\right)\left(\zeta_{\delta}^{\star}\right)^{2} \zeta+A_{2}^{-1} A_{3}\left(\zeta_{\delta}^{\star}\right)^{2} \bar{\zeta}-4 A_{2}^{-1} A_{4} \zeta_{\delta}^{\star} \vartheta_{\delta x}+\zeta_{\varepsilon, k}^{\dagger}\left(u^{\dagger}\right)+\tilde{\mathcal{R}}_{\varepsilon, k}(\zeta) \\
& \quad=A_{2}^{-1}\left(A_{3}+A_{5}\right)\left(\zeta^{\star}\right)^{2} \zeta+A_{2}^{-1} A_{3}\left(\zeta^{\star}\right)^{2} \bar{\zeta}-4 A_{2}^{-1} A_{4} \zeta^{\star} \vartheta_{x}+\zeta_{\varepsilon, k}^{\dagger}\left(u^{\dagger}\right)+\mathcal{R}_{\varepsilon, k}(\zeta),
\end{aligned}
$$

where

$$
\vartheta=A_{4} \mathcal{F}^{-1}\left[\frac{i \mu}{\left(1-\alpha_{0}^{-1}\right) \mu^{2}+k^{2}} \mathcal{F}\left[2 \operatorname{Re}\left(\zeta^{\star} \zeta\right)\right]\right]
$$

and

$$
\left\|\tilde{\mathcal{R}}_{\varepsilon, k}(\zeta)\right\|_{0},\left\|\mathcal{R}_{\varepsilon, k}(\zeta)\right\|_{0} \leq c \varepsilon\|\zeta\|_{1}
$$

In view of this calculation we now turn our attention to the solvability of the reduced equation

$$
\begin{aligned}
& A_{2}^{-1} \zeta-A_{2}^{-1} A_{1} \zeta_{x x} \\
& \quad-A_{2}^{-1}\left(A_{3}+A_{5}\right)\left(\zeta^{\star}\right)^{2} \zeta-A_{2}^{-1} A_{3}\left(\zeta^{\star}\right)^{2} \bar{\zeta}+4 A_{2}^{-1} A_{4} \zeta^{\star} \vartheta_{x}+k^{2} \zeta-\mathcal{R}_{\varepsilon, k}(\zeta)=\zeta^{\dagger}
\end{aligned}
$$

for arbitrary $\zeta^{\dagger} \in L^{2}(\mathbb{R})$ (for $k>0$ ) or $\zeta^{\dagger} \in L_{\mathrm{c}}^{2}(\mathbb{R})$ (for $k=0$ ). For $k>0$ we also note that each step in the reduction procedure preserves the invariance of the resolvent equations under the reflection $R$; in the present coordinates the action of this symmetry is given by $\zeta(x) \mapsto \overline{\zeta(-x)}$, so that $(62)$ is invariant under this transformation.

\section{Spectral theory for the reduced equation}

\subsection{Invertibility of the reduced operator}

In this section we reformulate the reduced equation (62) once more, reducing the question of its solvability to determining the location of an eigenvalue of an unbounded linear operator. We first present an auxiliary spectral result, one consequence of which is the solvability of (62) for $k=0$. 
Proposition 4.1 The formulae

$$
\begin{aligned}
& \mathcal{C}_{0,1}\left(\zeta_{1}\right)=A_{2}^{-1}\left(\zeta_{1}-A_{1} \zeta_{1 x x}-6 \operatorname{sech}^{2}\left(A_{1}^{-1 / 2} x\right) \zeta_{1}\right), \\
& \mathcal{C}_{0,2}\left(\zeta_{2}\right)=A_{2}^{-1}\left(\zeta_{2}-A_{1} \zeta_{2 x x}-2 \operatorname{sech}^{2}\left(A_{1}^{-1 / 2} x\right) \zeta_{2}\right)
\end{aligned}
$$

define

(i) self-adjoint operators $\mathcal{C}_{0,1}, \mathcal{C}_{0,2}: H^{2}(\mathbb{R}) \subseteq L^{2}(\mathbb{R}) \rightarrow L^{2}(\mathbb{R})$ whose spectrum consists of essential spectrum $\left[A_{2}^{-1}, \infty\right)$ and respectively two simple eigenvalues at $-3 A_{2}^{-1}, 0$ and a simple eigenvalue at 0 ;

(ii) self-adjoint operators $\mathcal{C}_{0,1}: H_{\mathrm{e}}^{2}(\mathbb{R}) \subseteq L_{\mathrm{e}}^{2}(\mathbb{R}) \rightarrow L_{\mathrm{e}}^{2}(\mathbb{R}), \mathcal{C}_{0,2}: H_{\mathrm{o}}^{2}(\mathbb{R}) \subseteq L_{\mathrm{o}}^{2}(\mathbb{R}) \rightarrow L_{\mathrm{o}}^{2}(\mathbb{R})$ whose spectrum consists of essential spectrum $\left[A_{2}^{-1}, \infty\right)$ and, in the case of $\mathcal{C}_{0,1}$, a simple eigenvalue at $-3 A_{2}^{-1}$.

Proof. The spectra of the operators $1-\partial_{x}^{2}-6 \operatorname{sech}^{2}(x): H^{2}(\mathbb{R}) \subseteq L^{2}(\mathbb{R}) \rightarrow L^{2}(\mathbb{R})$ and $1-\partial_{x}^{2}-2 \operatorname{sech}^{2}(x): H^{2}(\mathbb{R}) \subseteq L^{2}(\mathbb{R}) \rightarrow L^{2}(\mathbb{R})$ consists of essential spectrum $[1, \infty)$ and respectively two simple eigenvalues at -3 and 0 (with corresponding eigenvectors $\operatorname{sech}^{2}(x)$ and $\operatorname{sech}^{\prime}(x)$ ) and a simple eigenvalue at 0 (with corresponding eigenvector $\operatorname{sech}(x)$ ) (see Drazin [9. Chapter 4.11]). The assertions follow by a scaling argument and restricting to respectively even and odd functions.

Lemma 4.2 Suppose that $k=0$. The reduced equation $(62)$ has a unique solution $\zeta \in H_{\mathrm{c}}^{2}(\mathbb{R})$ for each $\zeta^{\dagger} \in L_{\mathrm{c}}^{2}(\mathbb{R})$.

Proof. Write equation (62) as

$$
\mathcal{C}_{\varepsilon}(\zeta)=\zeta^{\dagger}
$$

where

$$
\mathcal{C}_{\varepsilon}=\mathcal{C}_{0}+\mathcal{R}_{\varepsilon}, \quad \mathcal{C}_{0}(\zeta)=\left(\mathcal{C}_{0,1}\left(\zeta_{1}\right), \mathcal{C}_{0,2}\left(\zeta_{2}\right)\right)
$$

and $\zeta_{1}=\operatorname{Re} \zeta, \zeta_{2}=\operatorname{Im} \zeta$, and observe that $\mathcal{C}_{0}: H_{\mathrm{c}}^{2}(\mathbb{R}) \rightarrow L_{\mathrm{c}}^{2}(\mathbb{R})$ is invertible (see Proposition 4.1(ii)) and $\left\|\mathcal{R}_{\varepsilon}(\zeta)\right\|_{0} \leq c \varepsilon\|\zeta\|_{2}$.

Let $W$ be the real Hilbert space $L^{2}(\mathbb{R}) \times L^{2}(\mathbb{R}) \times L^{2}(\mathbb{R})$ equipped with the inner product

$$
\left\langle\left\langle\left(\zeta_{1}, \zeta_{2}, \psi\right),\left(\tilde{\zeta}_{1}, \tilde{\zeta}_{2}, \tilde{\psi}\right)\right\rangle=\left\langle\zeta_{1}, \tilde{\zeta}_{1}\right\rangle_{0}+\left\langle\zeta_{2}, \tilde{\zeta}_{2}\right\rangle_{0}+2 A_{2}^{-1}\langle\psi, \tilde{\psi}\rangle_{0}\right.
$$

and define unbounded operators $\mathcal{B}_{\varepsilon, k}, \tilde{\mathcal{B}}_{\varepsilon, k}: \mathcal{D}_{\mathcal{B}} \subseteq W \rightarrow W$ by the formulae

$$
\tilde{\mathcal{B}}_{\varepsilon, k}\left(\begin{array}{c}
\zeta_{1} \\
\zeta_{2} \\
\psi
\end{array}\right)=\left(\begin{array}{c}
A_{2}^{-1} \zeta_{1}-A_{2}^{-1} A_{1} \zeta_{1 x x}-A_{2}^{-1}\left(2 A_{3}+A_{5}\right)\left(\zeta^{\star}\right)^{2} \zeta_{1}+4 A_{2}^{-1} A_{4} \zeta^{\star} \psi_{x}-\operatorname{Re} \mathcal{R}_{\varepsilon, k}(\zeta) \\
A_{2}^{-1} \zeta_{2}-A_{2}^{-1} A_{1} \zeta_{2 x x}-A_{2}^{-1} A_{5}\left(\zeta^{\star}\right)^{2} \zeta_{2}-\operatorname{Im} \mathcal{R}_{\varepsilon, k}(\zeta) \\
-\left(1-\alpha_{0}^{-1}\right) \psi_{x x}-2 A_{4}\left(\zeta^{\star} \zeta_{1}\right)_{x}
\end{array}\right)
$$

and

$$
\mathcal{B}_{\varepsilon, k}=\tilde{\mathcal{B}}_{\varepsilon, k}+k^{2} I
$$

where $\mathcal{D}_{\mathcal{B}}:=H^{2}(\mathbb{R}) \times H^{2}(\mathbb{R}) \times H^{2}(\mathbb{R})$. In this framework equation 62 may be written as

$$
\mathcal{B}_{\varepsilon, k}\left(\zeta_{1}, \zeta_{2}, \psi\right)=\left(\operatorname{Re} \zeta^{\dagger}, \operatorname{Im} \zeta^{\dagger}, 0\right),
$$

where $\zeta_{1}=\operatorname{Re} \zeta, \zeta_{2}=\operatorname{Im} \zeta$; recall that this equation is invariant under the transformation $\tilde{R}:\left(\zeta_{1}(x), \zeta_{2}(x), \psi(x)\right)=\left(\zeta_{1}(-x),-\zeta_{2}(-x),-\psi(-x)\right)$ (the action of the reflection $R$ ).

The spectra of the self-adjoint operators $\mathcal{B}_{0, k}$ and $\tilde{\mathcal{B}}_{0, k}$ (the second of which does not depend upon $k$ ) can be determined precisely. 
Lemma 4.3 The spectrum of the operator $\tilde{\mathcal{B}}_{0, k}$ consists of essential spectrum $[0, \infty)$ and a simple negative eigenvalue $-k_{0}^{2}$ whose eigenspace lies in $\operatorname{Fix} \tilde{R}=L_{\mathrm{e}}^{2}(\mathbb{R}) \times L_{\mathrm{o}}^{2}(\mathbb{R}) \times L_{\mathrm{o}}^{2}(\mathbb{R})$.

Proof. First note that $\tilde{\mathcal{B}}_{0, k}$ is a compact perturbation of the constant-coefficient operator $\mathcal{D}_{\mathcal{B}} \subseteq$ $W \rightarrow W$ defined by

$$
\left(\zeta_{1}, \zeta_{2}, \psi\right) \mapsto\left(A_{2}^{-1} \zeta_{1}-A_{2}^{-1} A_{2} \zeta_{1 x x}, A_{2}^{-1} \zeta_{2}-A_{2}^{-1} A_{1} \zeta_{2 x x},-\left(1-\alpha_{0}^{-1}\right) \psi_{x x}\right),
$$

whose essential spectrum is clearly $[0, \infty)$; it follows that $\sigma_{\text {ess }}\left(\tilde{\mathcal{B}}_{0, k}\right)=[0, \infty)$ (see Kato, [21, Chapter IV, Theorem 5.26]). Because $\tilde{\mathcal{B}}_{0, k}$ is self-adjoint the remainder of its spectrum consists of negative real eigenvalues with finite multiplicity. Write $W^{1}=L^{2}(\mathbb{R}) \times L^{2}(\mathbb{R}), \mathcal{D}_{B}^{1}=H^{2}(\mathbb{R}) \times$ $H^{2}(\mathbb{R})$ and $W^{2}=L^{2}(\mathbb{R}), \mathcal{D}_{B}^{2}=H^{2}(\mathbb{R})$ and observe that (with a slight abuse of notation)

$$
\tilde{\mathcal{B}}_{0, k}\left(\zeta_{1}, \zeta_{2}, \psi\right)=\left(\tilde{\mathcal{B}}_{0, k, 1}\left(\zeta_{1}, \psi\right), \tilde{\mathcal{B}}_{0, k, 2} \zeta_{2}\right),
$$

where $\tilde{\mathcal{B}}_{0, k, 1}: \mathcal{D}_{\mathcal{B}}^{1} \subseteq W^{1} \rightarrow W^{1}$ and $\tilde{\mathcal{B}}_{0, k, 2}: \mathcal{D}_{\mathcal{B}}^{2} \subseteq W^{2} \rightarrow W^{2}$ are given by

$$
\tilde{\mathcal{B}}_{0, k, 1}\left(\begin{array}{c}
\zeta_{1} \\
\psi
\end{array}\right)=\left(\begin{array}{c}
A_{2}^{-1} \zeta_{1}-A_{2}^{-1} A_{1} \zeta_{1 x x}-A_{2}^{-1}\left(2 A_{3}+A_{5}\right)\left(\zeta^{\star}\right)^{2} \zeta_{1}+4 A_{2}^{-1} A_{4} \zeta^{\star} \psi_{x} \\
-\left(1-\alpha_{0}^{-1}\right) \psi_{x x}-2 A_{4}\left(\zeta^{\star} \zeta_{1}\right)_{x}
\end{array}\right)
$$

and

$$
\tilde{\mathcal{B}}_{0, k, 2} \zeta_{2}=\mathcal{C}_{0,2} \zeta_{2}
$$

the eigenvalues of $\tilde{\mathcal{B}}_{0, k}$ are therefore precisely the eigenvalues of $\tilde{\mathcal{B}}_{0, k, 1}$ and $\tilde{\mathcal{B}}_{0, k, 2}$.

According to Proposition 4.1 the operator $\tilde{\mathcal{B}}_{0, k, 2}$ has no negative eigenvalues. Turning to the spectrum of $\tilde{\mathcal{B}}_{0, k, 1}$, one finds by an explicit calculation that

$$
\left\langle\tilde{\mathcal{B}}_{0, k, 1}\left(\zeta_{1}, \psi\right),\left(\zeta_{1}, \psi\right)\right\rangle_{W^{1}}=\left\langle\mathcal{C}_{0,1} \zeta_{1}, \zeta_{1}\right\rangle_{0}+2 A_{2}^{-1}\left(1-\alpha_{0}^{-1}\right) \int_{\mathbb{R}}\left(\psi_{x}+\frac{2 A_{4}}{1-\alpha_{0}^{-1}} \zeta^{\star} \zeta_{1}\right)^{2} \mathrm{~d} x
$$

which quantity is positive for $\left(\zeta_{1}, \psi\right) \in W_{+}^{1}$, where

$$
W_{+}^{1}=\left\{\left(\zeta_{1}, \psi\right) \in W^{1}:\left\langle\left(\zeta_{1}, \psi\right),\left(\left(\operatorname{sech}^{2}\left(A_{1}^{-1 / 2} x\right), 0\right)\right\rangle_{W^{1}}=0\right\} .\right.
$$

It follows that any subspace of $W^{1}$ upon which $\tilde{\mathcal{B}}_{0, k, 1}$ is strictly negative definite is one-dimensional. The calculation

$$
\left\langle\tilde{\mathcal{B}}_{0, k, 1}\left(\left(\operatorname{sech}\left(A_{1}^{-1 / 2} x\right), 0\right),\left(\operatorname{sech}\left(A_{1}^{-1 / 2} x\right), 0\right)\right\rangle_{W^{1}}=-\frac{16 \sqrt{A_{1}} A_{3}}{3 A_{2} A_{5}}<0\right.
$$

shows that inf $\sigma\left(\tilde{\mathcal{B}}_{0, k, 1}\right)<0$, so that the spectral subspace of $W^{1}$ corresponding to the part of the spectrum of $\tilde{\mathcal{B}}_{0, k, 1}$ in $(-\infty,-\varepsilon)$ is nontrivial and hence one-dimensional for every sufficiently small value of $\varepsilon>0$. We conclude that $\tilde{\mathcal{B}}_{0, k, 1}$ has precisely one simple negative eigenvalue $-k_{0}^{2}$.

Finally, the same argument shows that $\left.\tilde{\mathcal{B}}_{0, k}\right|_{\text {Fix } \tilde{R}}$ also has precisely one simple negative eigenvalue. It follows that this eigenvalue is $-k_{0}^{2}$, whose eigenspace therefore lies in Fix $\tilde{R}$.

Noting that

$$
\left\|\tilde{\mathcal{B}}_{\varepsilon, k}-\tilde{\mathcal{B}}_{0, k}\right\|_{\mathcal{L}\left(\mathcal{D}_{\mathcal{B}}, W\right)} \leq c \varepsilon,
$$

we now use a perturbation argument to obtain a qualitative description of a portion of the spectrum of $\tilde{\mathcal{B}}_{\varepsilon, k}$. 
Lemma 4.4 Let $m$ and $M$ be positive real numbers with $m<k_{0}^{2}<M$ and $\gamma$ be an ellipse in the complex plane with major axis $[-M,-m]$. For each $k \in\left[0, k_{\max }\right]$ the portion of the spectrum of $\tilde{\mathcal{B}}_{\varepsilon, k}$ within $\gamma$ consists of precisely one simple real eigenvalue $\kappa_{\varepsilon, k}$ with $\kappa_{0, k}=-k_{0}^{2}$; its eigenspace lies in Fix $\tilde{R}$. In particular, $\tilde{\mathcal{B}}_{\varepsilon, k}$ is closed and $\tilde{\mathcal{B}}_{\varepsilon, k}-\kappa I: \mathcal{D}_{\mathcal{B}} \rightarrow W$ is Fredholm with index 0 for $k \in\left[0, k_{\max }\right]$ and $\kappa \in[-M,-m]$.

Proof. The contour $\gamma$ defines a separation of $\sigma\left(\tilde{\mathcal{B}}_{0, k}\right)$ : the portion of $\sigma\left(\tilde{\mathcal{B}}_{0, k}\right)$ in the interior of $\gamma$ consists of precisely one simple eigenvalue $-k_{0}^{2}$ and the remainder of $\sigma\left(\tilde{\mathcal{B}}_{0, k}\right)$ lies in the exterior of $\gamma$. In view of inequality $\sqrt{63}$ and the fact that $\tilde{\mathcal{B}}_{0, k}$ does not depend upon $k$, we may apply a standard argument in spectral perturbation theory (see Kato [21, Theorem 3.16]), which asserts that $\gamma$ defines the same separation of $\tilde{\mathcal{B}}_{\varepsilon, k}$. Since $\tilde{\mathcal{B}}_{\varepsilon, k}$ is a real operator its eigenvalues arise in complex-conjugate pairs; its simple eigenvalue $\kappa_{\varepsilon, k}$ in the interior of $\gamma$ is therefore real. The same argument applies to $\left.\tilde{\mathcal{B}}_{\varepsilon, k}\right|_{\text {Fix } \tilde{R}}$, whence the eigenspace corresponding to $\kappa_{\varepsilon, k}$ lies in Fix $\tilde{R}$.

This argument implies in particular that $\tilde{\mathcal{B}}_{\varepsilon, k}$ is closed and that $\tilde{\mathcal{B}}_{\varepsilon, k}-\kappa I: \mathcal{D}_{\mathcal{B}} \rightarrow W$ is Fredholm with index 0 for $\kappa \in[-M,-m]$ (because $[-M,-m] \backslash\left\{\kappa_{\varepsilon, k}\right\} \subseteq \rho\left(\tilde{\mathcal{B}}_{\varepsilon, k}\right)$ and $\kappa_{\varepsilon, k}$ is a simple eigenvalue of $\tilde{\mathcal{B}}_{\varepsilon, k}$ ).

Our next result is deduced from inequality (63) using the method given by Groves, Haragus \& Sun [14, Lemma 3.15].

Lemma 4.5 The spectral projection $P_{\varepsilon, k} \in \mathcal{L}\left(W, \mathcal{D}_{\mathcal{B}}\right)$ defined by

$$
P_{\varepsilon, k} w=\frac{1}{2 \pi \mathrm{i}} \int_{\gamma}\left(\kappa I-\tilde{\mathcal{B}}_{\varepsilon, k}\right)^{-1} w \mathrm{~d} \kappa
$$

satisfies the estimate

$$
\left\|P_{\varepsilon, k}-P_{0, k}\right\|_{\mathcal{L}\left(W, \mathcal{D}_{\mathcal{B}}\right)} \leq c \varepsilon
$$

uniformly in $k \in\left[0, k_{\max }\right]$.

Finally, choose $k_{\max }>k_{0}$ and $k_{\min } \in\left(0, k_{0}\right)$ and apply Lemma 4.4 , choosing $m, M$ such that $M>k_{\max }^{2}$ and $m<k_{\min }^{2}$, so that the point $-k^{2}$ lies in the interior of $\gamma$ for all $k \in\left[k_{\min }, k_{\max }\right]$ (see Figure 3). The following lemma follows from the relation $\mathcal{B}_{\varepsilon, k}=\tilde{\mathcal{B}}_{\varepsilon, k}+k^{2} I$.

Lemma 4.6 Fix $k \in\left[k_{\min }, k_{\max }\right]$.

(i) The operator $\mathcal{B}_{\varepsilon, k}: \mathcal{D}_{\mathcal{B}} \subseteq W \rightarrow W$ is invertible provided that $\kappa_{\varepsilon, k}+k^{2} \neq 0$.

(ii) The operator $\mathcal{B}_{\varepsilon, k}: \mathcal{D}_{\mathcal{B}} \subseteq W \rightarrow W$ has a simple zero eigenvalue whenever $\kappa_{\varepsilon, k}+k^{2}=$ 0 .

In Section 4.3 below we show that the equation $\kappa_{\varepsilon, k}+k^{2}=0$ has precisely one solution $k_{\varepsilon}$ in the interval $\left[k_{\min }, k_{\max }\right]$ (see Figure 3 . 


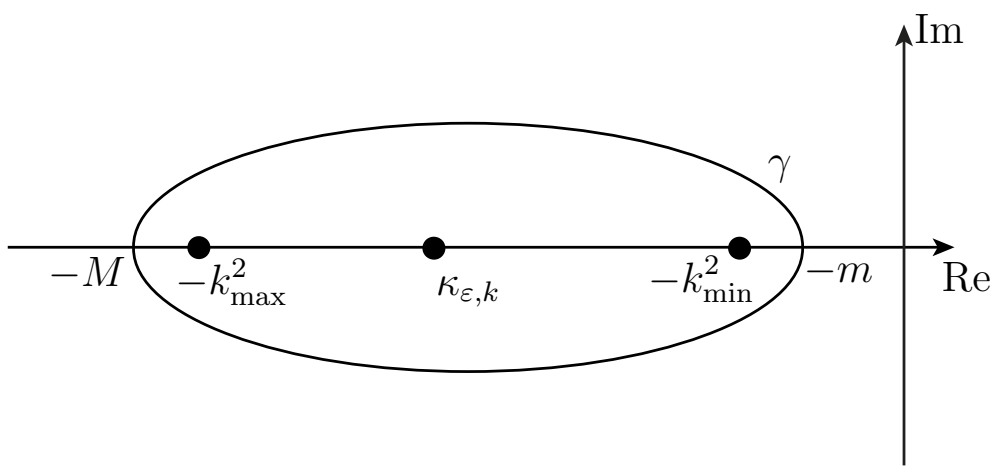

Figure 3: The portion of $\sigma\left(\tilde{\mathcal{B}}_{\varepsilon, k}\right)$ within $\gamma$ consists of precisely one simple real eigenvalue $\kappa_{\varepsilon, k} \in$ $\left[-k_{\max }^{2},-k_{\min }^{2}\right]$ with $\kappa_{0, k}=-k_{0}^{2}$.

\subsection{Small spectral values}

In this section we prove that $\left.\mathcal{B}_{\varepsilon, k}\right|_{\text {Fix } \tilde{R}}$ is semi-Fredholm and injective for $k \in\left(0, k_{\min }\right)$. Because this operator is closed (see Lemma 4.4) it suffices to establish the a priori estimate

$$
\|w\|_{W} \leq c k^{-2}\left\|\mathcal{B}_{\varepsilon, k} w\right\|_{W}, \quad k \in\left(0, k_{\min }\right),
$$

for sufficiently small values of $k_{\min }$. For notational simplicity we here abbreviate Fix $\tilde{R}$ and $\mathcal{D}_{\mathcal{B}} \cap$ Fix $\tilde{R}$ to respectively $W$ and $\mathcal{D}_{\mathcal{B}}$, so that $W=L_{\mathrm{e}}^{2}(\mathbb{R}) \times L_{\mathrm{o}}^{2}(\mathbb{R}) \times L_{\mathrm{o}}^{2}(\mathbb{R})$ and $\mathcal{D}_{\mathcal{B}}=$ $H_{\mathrm{e}}^{2}(\mathbb{R}) \times H_{\mathrm{o}}^{2}(\mathbb{R}) \times H_{\mathrm{o}}^{2}(\mathbb{R})$.

The first step is to choose an eigenvector corresponding to the eigenvalue $\kappa_{\varepsilon, k}$ of $\mathcal{B}_{\varepsilon, k}$ in a perturbative fashion.

Lemma 4.7 There exists an eigenvector $w_{\varepsilon, k}=\left(\zeta_{\varepsilon, k}, \psi_{\varepsilon, k}\right)$ of $\tilde{\mathcal{B}}_{\varepsilon, k}$ corresponding to the eigenvalue $\kappa_{\varepsilon, k}$ such that $\left\|w_{\varepsilon, k}\right\|_{W}=1$ and $\left\|w_{\varepsilon, k}-w_{0, k}\right\|_{\mathcal{D}_{\mathcal{B}}} \leq c \varepsilon$. Furthermore, there exists $a$ primitive $\Psi_{\varepsilon, k} \in H_{\mathrm{e}}^{3}(\mathbb{R})$ of $\psi_{\varepsilon, k}$ with $\left\|\Psi_{\varepsilon, k}-\Psi_{0, k}\right\|_{0} \leq c \varepsilon$.

Proof. Choose the eigenvector $w_{\varepsilon, k}$ using the formula

$$
w_{\varepsilon, k}=\left\|P_{\varepsilon, k} w_{0}\right\|_{W}^{-1} P_{\varepsilon, k} w_{0},
$$

where $w_{0}$ is a unit eigenvector of $\mathcal{B}_{k, 0}$ corresponding to the eigenvalue $-k_{0}^{2}$. (The calculation

$$
\begin{aligned}
\left\|P_{\varepsilon, k} w_{0}\right\|_{W} & =\left\|P_{0, k} w_{0}+\left(P_{\varepsilon, k}-P_{0, k}\right) w_{0}\right\|_{W} \\
& \geq\left\|w_{0}\right\|_{W}-\left\|\left(P_{\varepsilon, k}-P_{0, k}\right) w_{0}\right\|_{W} \\
& \geq 1-c \varepsilon
\end{aligned}
$$

shows that $P_{\varepsilon, k} w_{0} \neq 0$.) It follows that

$$
\begin{aligned}
\left\|w_{\varepsilon, k}-w_{0, k}\right\|_{\mathcal{D}_{\mathcal{B}}} & \leq\|\| P_{\varepsilon, k} w_{0}\left\|_{W}^{-1} P_{\varepsilon, k}-P_{0, k}\right\|_{\mathcal{L}\left(W, \mathcal{D}_{\mathcal{B}}\right)}\left\|w_{0, k}\right\|_{W} \\
& \leq\left|\left\|P_{\varepsilon, k} w_{0}\right\|_{W}^{-1}-1\right|\left\|P_{\varepsilon, k}\right\|_{\mathcal{L}\left(W, \mathcal{D}_{\mathcal{B}}\right)}+\left\|P_{\varepsilon, k}-P_{0, k}\right\|_{\mathcal{L}\left(W, \mathcal{D}_{\mathcal{B}}\right)} \\
& \leq c \varepsilon .
\end{aligned}
$$


Define

$$
\Psi_{\varepsilon, k}=-\kappa_{\varepsilon, k}^{-1}\left(1-\alpha_{0}^{-1}\right) \psi_{\varepsilon, k, x}-2 \kappa_{\varepsilon, k}^{-1} A_{4} \zeta^{\star} \zeta_{\varepsilon, k, 1}
$$

so that

$$
\begin{aligned}
\Psi_{\varepsilon, k, x} & =\kappa_{\varepsilon, k}^{-1}\left(-\left(1-\alpha_{0}^{-1}\right) \psi_{\varepsilon, k, x x}-2 A_{4}\left(\zeta^{\star} \zeta_{\varepsilon, k, 1}\right)_{x}\right) \\
& =\psi_{\varepsilon, k}
\end{aligned}
$$

and

$$
\begin{aligned}
\left\|\Psi_{\varepsilon, k}-\Psi_{\varepsilon, 0}\right\|_{0} & =\left\|-\left(1-\alpha_{0}^{-1}\right)\left(\frac{\psi_{\varepsilon, k, x}}{\kappa_{\varepsilon, k}}-\frac{\psi_{0, k, x}}{\kappa_{0, k}}\right)-2 A_{4}\left(\frac{\zeta_{\varepsilon, k, 1}}{\kappa_{\varepsilon, k}}-\frac{\zeta_{0, k, 1}}{\kappa_{0, k}}\right)\right\|_{0} \\
& \leq c\left\|\frac{w_{\varepsilon, k}}{\kappa_{\varepsilon, k}}-\frac{w_{0, k}}{\kappa_{0, k}}\right\|_{\mathcal{D}_{\mathcal{B}}} \\
& \leq c \varepsilon
\end{aligned}
$$

We now define the auxiliary operator $\mathcal{A}_{\varepsilon, k}: \mathcal{D}_{\mathcal{A}} \subseteq V \rightarrow V$ by the formula

$$
\mathcal{A}_{\varepsilon, k}\left(\begin{array}{c}
\zeta_{1} \\
\zeta_{2} \\
\phi
\end{array}\right)=\left(\begin{array}{c}
A_{2}^{-1} \zeta_{1}-A_{2}^{-1} A_{1} \zeta_{1 x x}-A_{2}^{-1}\left(2 A_{3}+A_{5}\right)\left(\zeta^{\star}\right)^{2} \zeta_{1}+4 A_{2}^{-1} A_{4} \zeta^{\star} \phi-\operatorname{Re} \mathcal{R}_{\varepsilon, k}(\zeta) \\
A_{2}^{-1} \zeta_{2}-A_{2}^{-1} A_{1} \zeta_{2 x x}-A_{2}^{-1} A_{5}\left(\zeta^{\star}\right)^{2} \zeta_{2}-\operatorname{Im} \mathcal{R}_{\varepsilon, k}(\zeta) \\
\left(1-\alpha_{0}^{-1}\right) \phi+2 A_{4} \zeta^{\star} \zeta_{1}
\end{array}\right)
$$

where $V=L_{\mathrm{e}}^{2}(\mathbb{R}) \times L_{\mathrm{o}}^{2}(\mathbb{R}) \times L_{\mathrm{e}}^{2}(\mathbb{R})$ and $\mathcal{D}_{\mathcal{A}}=H_{\mathrm{e}}^{2}(\mathbb{R}) \times H_{\mathrm{o}}^{2}(\mathbb{R}) \times L_{\mathrm{e}}^{2}(\mathbb{R})$. Observe that $\mathcal{A}_{0, k}$ is self-adjoint with respect to the inner product $\langle\langle\cdot, \cdot\rangle\rangle$ for $V$, and repeating the arguments used in the proof of Lemma 4.3 , one finds that its spectrum consists of essential spectrum $\{1-$ $\left.\alpha_{0}^{-1}\right\} \cup\left[A_{2}^{-1}, \infty\right)$ and a simple negative eigenvalue $-\omega_{1}^{2}$ (the point $1-\alpha_{0}^{-1}$ is an eigenvalue with infinite-dimensional eigenspace $\left.\{(0,0)\} \times L_{\mathrm{e}}^{2}(\mathbb{R})\right)$. Below we write $v=\left(\zeta, \psi_{x}\right) \in \mathcal{D}_{\mathcal{A}}$ for $w=(\zeta, \psi) \in \mathcal{D}_{\mathcal{B}}$ and in particular set $v_{\varepsilon, k}=\left(\zeta_{\varepsilon, k}, \psi_{\varepsilon, k, x}\right)$.

Lemma 4.8 The operator $\mathcal{A}_{0, k}: \mathcal{D}_{\mathcal{A}} \subseteq V \rightarrow V$ satisfies

$$
\left\langle\left\langle\mathcal{A}_{0, k} v_{0, k}, v_{0, k}\right\rangle\right\rangle=-k_{0}^{2}, \quad\left\langle\left\langle\mathcal{A}_{0, k} v_{0, k}, v\right\rangle\right\rangle=0, \quad\left\langle\left\langle\mathcal{A}_{0, k} v, v\right\rangle\right\rangle \geq c\|v\|_{V}^{2}
$$

for each $w \in \mathcal{D}_{\mathcal{B}}$ with $\left\langle\left\langle w, w_{0, k}\right\rangle\right\rangle=0$.

Proof. Note that

$$
\mathcal{A}_{0, k}\left(\zeta_{0, k}, \psi_{0, k, x}\right)=-k_{0}^{2}\left(\zeta_{0, k},-\Psi_{0, k}\right)
$$

and therefore that

$$
\begin{aligned}
\left\langle\left\langle\mathcal{A}_{0, k} v_{0, k}, v_{0, k}\right\rangle\right\rangle & =-k_{0}^{2}\left\langle\left\langle\left(\zeta_{0, k},-\Psi_{0, k}\right),\left(\zeta_{0, k}, \phi_{0, k}\right)\right\rangle\right\rangle \\
& =-k_{0}^{2}\left(\left\|\zeta_{0, k}\right\|_{0}^{2}-2 A_{2}^{-1}\left\langle\Psi_{0, k}, \psi_{0, k, x}\right\rangle_{0}\right) \\
& =-k_{0}^{2}\left\|w_{0, k}\right\|_{V}^{2} \\
& =-k_{0}^{2}
\end{aligned}
$$


and

$$
\begin{aligned}
\left.\left\langle\mathcal{A}_{0, k} v_{0, k}, v\right\rangle\right\rangle & =-k_{0}^{2}\left\langle\left\langle\left(\zeta_{0, k},-\Psi_{0, k}\right),\left(\zeta, \psi_{x}\right)\right\rangle\right\rangle \\
& =-k_{0}^{2}\left(\left\langle\zeta_{0, k}, \zeta\right\rangle_{0}-2 A_{2}^{-1}\left\langle\Psi_{0, k}, \psi_{x}\right\rangle_{0}\right) \\
& =-k_{0}^{2}\left\langle\left\langle w_{0, k}, w\right\rangle\right\rangle \\
& =0 .
\end{aligned}
$$

Let $\left\{P_{\kappa}\right\}_{\kappa \in \mathbb{R}}$ denote the spectral family of $\mathcal{A}_{\varepsilon, k}$ and write

$$
v_{0, k}=c_{0} e+\int_{1-\alpha_{0}^{-1}}^{\infty} \mathrm{d}\left(P_{\kappa} v_{0, k}\right), \quad v=d_{0} e+\int_{1-\alpha_{0}^{-1}}^{\infty} \mathrm{d}\left(P_{\kappa} v\right) .
$$

where $e$ is a unit eigenvector corresponding to the negative eigenvalue, $c_{0}=\left\langle\left\langle v_{0, k}, e\right\rangle\right\rangle$ and $d_{0}=$ $\langle\langle v, e\rangle\rangle$, so that

$$
\begin{aligned}
\left\langle\left\langle\mathcal{A}_{0, k} v_{0, k}, v_{0, k}\right\rangle\right\rangle & =-\omega_{1}^{2} c_{0}^{2}+\int_{1-\alpha_{0}^{-1}}^{\infty} \kappa \mathrm{d}\left(\left\langle\left\langle P_{\kappa} v_{0, k}, v_{0, k}\right\rangle\right\rangle\right), \\
\left\langle\left\langle\mathcal{A}_{0, k} v, v\right\rangle\right\rangle & =-\omega_{1}^{2} d_{0}^{2}+\int_{1-\alpha_{0}^{-1}}^{\infty} \kappa \mathrm{d}\left(\left\langle P_{\kappa} v, v\right\rangle\right), \\
\left.\left\langle\mathcal{A}_{0, k} v_{0, k}, v\right\rangle\right\rangle & =-\omega_{1}^{2} c_{0} d_{0}+\int_{1-\alpha_{0}^{-1}}^{\infty} \kappa \mathrm{d}\left(\left\langle\left\langle P_{\kappa} v_{0, k}, v\right\rangle\right\rangle\right) .
\end{aligned}
$$

It follows from 64) and 66 that

$$
c_{0}^{2}=\frac{1}{\omega_{1}^{2}}\left(k_{0}^{2}+\int_{1-\alpha_{0}^{-1}}^{\infty} \kappa \mathrm{d}\left(\left\langle\left\langle P_{\kappa} v_{0, k}, v_{0, k}\right\rangle\right\rangle\right)\right) \neq 0,
$$

and equations (65) and (68) imply that

$$
\left.\omega_{1} d_{0}=\frac{1}{\omega_{1} c_{0}} \int_{1-\alpha_{0}^{-1}}^{\infty} \kappa \mathrm{d}\left(\left\langle P_{\kappa} v_{0, k}, v\right\rangle\right\rangle\right) .
$$

The estimate

$$
\begin{aligned}
\left\langle\left\langle\mathcal{A}_{0, k} v, v\right\rangle\right\rangle & =\frac{1}{c_{0}^{2} \omega_{1}^{2}}\left\{-\left(\int_{1-\alpha_{0}^{-1}}^{\infty} \kappa \mathrm{d}\left(\left\langle P_{\kappa} v_{0, k}, v\right\rangle\right)\right)^{2}\right. \\
& \left.\quad+\left(k_{0}^{2}+\int_{1-\alpha_{0}^{-1}}^{\infty} \kappa \mathrm{d}\left(\left\langle P_{\kappa} v_{0, k}, v_{0, k}\right\rangle\right\rangle\right)\right) \int_{1-\alpha_{0}^{-1}}^{\infty} \kappa \mathrm{d}\left(\left\langle\left\langle P_{\kappa} v, v\right\rangle\right)\right\} \\
\geq & \left.\frac{k_{0}^{2}}{c_{0}^{2} \omega_{1}^{2}} \int_{1-\alpha_{0}^{-1}}^{\infty} \kappa \mathrm{d}\left(\left\langle P_{\kappa} v, v\right\rangle\right\rangle\right) \\
\geq & 0
\end{aligned}
$$

shows in particular that

$$
d_{0}^{2} \leq \frac{1}{\omega_{1}^{2}} \int_{1-\alpha_{0}^{-1}}^{\infty} \kappa \mathrm{d}\left(\left\langle P_{\kappa} v, v\right\rangle\right)
$$


(see equation (67)), whence

$$
\begin{aligned}
\|v\|_{V}^{2} & =d_{0}^{2}+\int_{1-\alpha_{0}^{-1}}^{\infty} \mathrm{d}\left(\left\langle P_{\kappa} v, v\right\rangle\right) \\
& \leq\left(\frac{1}{\omega_{1}^{2}}+\frac{1}{1-\alpha_{0}^{-1}}\right) \int_{1-\alpha_{0}^{-1}}^{\infty} \kappa \mathrm{d}\left(\left\langle\left\langle P_{\kappa} v, v\right\rangle\right\rangle\right) \\
& \leq \frac{\omega_{1}^{2} c_{0}^{2}}{k_{0}^{2}}\left(\frac{1}{\omega_{1}^{2}}+\frac{1}{1-\alpha_{0}^{-1}}\right)\left\langle\left\langle\mathcal{A}_{0, k} v, v\right\rangle .\right.
\end{aligned}
$$

The a priori estimate announced at the start of this section (Theorem 4.10 below) is a corollary of the following lemma.

Lemma 4.9 The operator $\mathcal{B}_{\varepsilon, k}: \mathcal{D}_{\mathcal{B}} \subseteq W \rightarrow W$ satisfies the estimate

$$
\left\langle\left\langle\mathcal{B}_{\varepsilon, k} w, w\right\rangle \geq c k^{2}\|w\|_{1}^{2}, \quad k \leq k_{\min }\right.
$$

for each $w \in \mathcal{D}_{\mathcal{B}}$ such that $\left\langle\left\langle w, w_{\varepsilon, k}\right\rangle\right\rangle=0$ and each sufficiently small value of $k_{\min }$.

Proof. Write

$$
w=a w_{0, k}+w^{\perp},
$$

where $a=\left\langle\left\langle w, w_{0, k}\right\rangle\right\rangle,\left\langle\left\langle w^{\perp}, w_{0, k}\right\rangle\right\rangle=0$ and note that

$$
\begin{aligned}
|a| & =\left|\left\langle\left\langle w, w_{0, k}\right\rangle\right\rangle\right| \\
& =\left|\left\langle\left\langle w, w_{0, k}-w_{\varepsilon, k}\right\rangle\right\rangle\right| \\
& =\left|\left\langle\left\langle v,\left(\left(\zeta_{0, k}-\zeta_{\varepsilon, k}\right),-\left(\Psi_{0, k}-\Psi_{\varepsilon, k}\right)\right)\right\rangle\right\rangle\right| \\
& \leq c \varepsilon\|v\|_{V} .
\end{aligned}
$$

Clearly

$$
v=a v_{0, k}+v^{\perp},
$$

so that

$$
\left\|v^{\perp}\right\|_{V}^{2}=\|v\|_{V}^{2}-2 a\left\langle\left\langle v, v_{0, k}\right\rangle\right\rangle+a^{2},
$$

and follows from Lemma 4.8 that

$$
\begin{aligned}
\left\langle\left\langle\mathcal{A}_{0, k} v, v\right\rangle\right\rangle & =a^{2}\left\langle\left\langle\mathcal{A}_{0, k} v_{0, k}, v_{0, k}\right\rangle+2 a\left\langle\left\langle\mathcal{A}_{0, k} v_{0, k}, v^{\perp}\right\rangle+\left\langle\left\langle\mathcal{A}_{0, k} v^{\perp}, v^{\perp}\right\rangle\right\rangle\right.\right. \\
& \geq c\left(\left\|v^{\perp}\right\|_{V}^{2}-a^{2}\right) \\
& \geq c\left(\|v\|_{V}^{2}-2 a\left\langle\left\langle v, v_{0, k}\right\rangle\right\rangle\right) \\
& \geq c\|v\|_{V}^{2}
\end{aligned}
$$

because

$$
\left|2 a\left\langle\left\langle v, v_{0, k}\right\rangle\right\rangle\right| \leq 2|a|\|v\|_{V}\left\|v_{0, k}\right\|_{V} \leq 2 c \varepsilon\|v\|_{V}^{2} .
$$


On the other hand

$$
\begin{aligned}
\left\langle\left\langle\mathcal{A}_{0, k} v, v\right\rangle\right\rangle= & \frac{1}{A_{2}} \int_{\mathbb{R}}\left(\zeta_{1}^{2}+A_{1} \zeta_{1 x}^{2}-6 \operatorname{sech}^{2}\left(A_{1}^{-1 / 2} x\right) \zeta_{1}^{2}\right) \mathrm{d} x \\
& +2 A_{2}^{-1}\left(1-\alpha_{0}^{-1}\right) \int_{\mathbb{R}}\left(\phi+\frac{2 A_{4}}{1-\alpha_{0}^{-1}} \zeta^{\star} \zeta_{1}\right)^{2} \mathrm{~d} x \\
& \quad+\frac{1}{A_{2}} \int_{\mathbb{R}}\left(\zeta_{1}^{2}+A_{1} \zeta_{1 x}^{2}-2 \operatorname{sech}^{2}\left(A_{1}^{-1 / 2} x\right) \zeta_{1}^{2}\right) \mathrm{d} x \\
\geq & \frac{A_{1}}{A_{2}}\left\|\zeta_{x}\right\|_{0}^{2}-c\|\zeta\|_{0}^{2},
\end{aligned}
$$

and combining the previous two estimates shows that

$$
\begin{aligned}
\left\langle\left\langle\mathcal{A}_{0, k} v, v\right\rangle\right\rangle & \geq \frac{\delta A_{1}}{A_{2}}\left\|\zeta_{x}\right\|^{2}-c \delta\|\zeta\|_{0}^{2}+(1-\delta) c\|v\|^{2} \\
& \geq c\left(\|\zeta\|_{1}^{2}+\|\phi\|_{0}^{2}\right)
\end{aligned}
$$

for sufficiently small $\delta>0$. Using the estimate

$$
\left\|\mathcal{R}_{\varepsilon, k}(\zeta)\right\|_{0} \leq c \varepsilon\|\zeta\|_{1}
$$

one finds that

$$
\begin{aligned}
\left\langle\left\langle\mathcal{A}_{\varepsilon, k} v, v\right\rangle\right\rangle & =\left\langle\left\langle\mathcal{A}_{0, k} v, v\right\rangle\right\rangle-\left\langle\left\langle\left(\mathcal{R}_{\varepsilon, k}(\zeta), 0\right),(\zeta, 0)\right\rangle\right\rangle \\
& \geq c\left(\|\zeta\|_{1}^{2}+\|\phi\|_{0}^{2}\right)
\end{aligned}
$$

We conclude that

$$
\begin{aligned}
\left\langle\left\langle\mathcal{B}_{\varepsilon, k} w, w\right\rangle\right. & =\left\langle\left\langle\mathcal{A}_{\varepsilon, k} v, v\right\rangle+k^{2}\langle\langle w, w\rangle\rangle\right. \\
& \geq c\left(\|\zeta\|_{1}^{2}+\left\|\psi_{x}\right\|_{0}^{2}\right)+k^{2}\left(\|\zeta\|_{0}^{2}+\|\psi\|_{0}^{2}\right) \\
& \geq k^{2}\left(\|\zeta\|_{1}^{2}+\|\psi\|_{1}^{2}\right)
\end{aligned}
$$

for $k<k_{\min }$ and $k_{\min }$ sufficiently small.

Theorem 4.10 Choose $k \in\left(0, k_{\min }\right]$ and $w^{\dagger} \in W$. Any solution $w \in \mathcal{D}_{\mathcal{B}}$ of the equation

$$
\mathcal{B}_{\varepsilon, k} w=w^{\dagger}
$$

satisfies the estimate

$$
\|w\|_{W} \leq c k^{-2}\left\|w^{\dagger}\right\|_{W}
$$

Proof. Write

$$
w=c_{\varepsilon, k} w_{\varepsilon, k}+w^{\perp}, \quad w^{\dagger}=d_{\varepsilon, k} w_{\varepsilon, k}+\left(w^{\dagger}\right)^{\perp},
$$

where $c_{\varepsilon, k}=\left\langle\left\langle w, w_{\varepsilon, k}\right\rangle\right\rangle, d_{\varepsilon, k}=\left\langle\left\langle w^{\dagger}, w_{\varepsilon, k}\right\rangle\right\rangle$ and $\left\langle\left\langle w^{\perp}, w_{\varepsilon, k}\right\rangle\right\rangle=0,\left\langle\left\langle w^{\dagger}, w_{\varepsilon, k}\right\rangle\right\rangle=0$, so that

$$
c_{\varepsilon, k}\left(\kappa_{\varepsilon, k}+k^{2}\right) w_{\varepsilon, k}+\mathcal{B}_{\varepsilon, k} w^{\perp}=d_{\varepsilon, k} w_{\varepsilon, k}+\left(w^{\dagger}\right)^{\perp} .
$$


Taking the inner product of equation 69 with $w^{\perp}$, we find that

$$
\left\langle\left\langle\mathcal{B}_{\varepsilon, k} w^{\perp}, w^{\perp}\right\rangle\right\rangle=\left\langle\left\langle\left(w^{\dagger}\right)^{\perp}, w^{\perp}\right\rangle,\right.
$$

and it follows from Lemma 4.9 that

$$
\left\|w^{\perp}\right\|_{1} \leq \frac{c}{k^{2}}\left\|\left(w^{\dagger}\right)^{\perp}\right\|_{W} .
$$

On the other hand, taking the inner product of (69) with $w_{\varepsilon, k}$ yields

$$
\left.\left|c_{\varepsilon, k}\right|=\frac{1}{\left|\kappa_{\varepsilon, k}+k^{2}\right|}\left(\left|d_{\varepsilon, k}\right|+\left|\left\langle\mathcal{B}_{0, k} w^{\perp}, w_{\varepsilon, k}\right\rangle\right\rangle|+|\left\langle\mathcal{R}_{\varepsilon, k}\left(\zeta^{\perp}\right), \zeta_{\varepsilon, k}\right\rangle\right\rangle \mid\right),
$$

where $w^{\perp}=\left(\zeta^{\perp}, \psi^{\perp}\right)$, because $\mathcal{B}_{\varepsilon, k}\left(w^{\perp}\right)=\mathcal{B}_{0, k}\left(w^{\perp}\right)+\left(\mathcal{R}_{\varepsilon, k}\left(\zeta^{\perp}\right), 0\right)$. Estimating

$$
\left|\left\langle\mathcal{B}_{0, k} w^{\perp}, w_{\varepsilon, k}\right\rangle\right| \leq c\left\|w^{\perp}\right\|_{1}\left\|w_{\varepsilon, k}\right\|_{1},
$$

where we have integrated by parts,

$$
\mid\left\langle\left\langle\mathcal{R}_{\varepsilon, k}\left(\zeta^{\perp}\right), \zeta_{\varepsilon, k}\right\rangle\right| \leq\left\|\mathcal{R}_{\varepsilon, k}\left(\zeta^{\perp}\right)\right\|_{0}\left\|\zeta_{\varepsilon, k}\right\|_{0} \leq c\left\|\zeta^{\perp}\right\|_{1}\left\|\zeta_{\varepsilon, k}\right\|_{0}
$$

and

$$
\left|\kappa_{\varepsilon, k}+k^{2}\right| \geq k_{0}^{2}-k_{\min }^{2}>0
$$

we find that

$$
\left|c_{\varepsilon, k}\right| \leq c\left(\left|d_{\varepsilon, k}\right|+\left\|w^{\perp}\right\|_{1}\right)
$$

Estimates (70) and (71) show that

$$
\begin{aligned}
\|w\|_{V} & =\left|c_{\varepsilon, k}\right|+\left\|w^{\perp}\right\|_{V} \\
& \leq c\left(\left|d_{\varepsilon, k}\right|+\frac{1}{k^{2}}\left\|\left(w^{\dagger}\right)^{\perp}\right\|_{V}\right) \\
& \leq \frac{c}{k^{2}}\left\|w^{\perp}\right\|_{V} .
\end{aligned}
$$

\subsection{Solution of the eigenvalue equation}

In this section we determine the solution set of the equation

$$
\kappa_{\varepsilon, k}+k^{2}=0
$$

in the interval $\left[k_{\min }, k_{\max }\right]$. The solution set is known explicitly for $\varepsilon=0$, and we approach the problem for $\varepsilon>0$ using a perturbation argument. Inequality (63) yields the necessary estimate for $\kappa_{\varepsilon, k}$, and to obtain the corresponding estimate for $\kappa_{\varepsilon, k}^{\prime}$ we show that the linear mapping $\mathcal{R}_{\varepsilon, k}: H^{2}(\mathbb{R}) \rightarrow L^{2}(\mathbb{R})$ is a continuously differentiable function of $k>0$ which satisfies the estimate

$$
\left\|\mathcal{R}_{\varepsilon, k}(\zeta)^{\prime}\right\|_{0} \leq c \varepsilon
$$

uniformly over $k \in\left[k_{\min }, k_{\max }\right]$ (which implies the corresponding result for $\tilde{\mathcal{B}}_{\varepsilon, k}$ ). This property of $\mathcal{R}_{\varepsilon, k}$ is established by systematically examining each step in its derivation in Section 3 and checking the continuous differentiability of the operators appearing there. 
Proposition 4.11 The linear mappings $\tilde{\Gamma}(\cdot, 0), \tilde{\Gamma}^{(j)}, j=1,2,3$, and $\tilde{\Gamma}^{\mathrm{r}}: H^{2}(\mathbb{R}) \rightarrow H^{2}(\Sigma)$ are continuously differentiable functions of $k>0$.

Proof. Recall that

$$
\tilde{\Gamma}\left(\eta, u^{\dagger}\right)=\mathcal{G}^{\star}\left(\tilde{\Gamma}\left(\eta, u^{\dagger}\right), \eta, u^{\dagger}\right)
$$

where

$$
\mathcal{G}^{\star}\left(\eta, \Gamma, u^{\dagger}\right)=\mathcal{G}_{1}\left(F_{1}(\eta, \Gamma), F_{2}(\eta, \Gamma), F_{3}(\eta, \Gamma), \eta\right)+\mathcal{G}_{2}\left(F^{\dagger}\left(u^{\dagger}\right)\right)
$$

so that

$$
\tilde{\Gamma}(\eta, 0)=\left(I-\mathcal{G}^{\star}(0, \cdot, 0)\right)^{-1} \mathcal{G}^{\star}(0, \eta, 0) .
$$

This formula and the fact that $k \mapsto \mathcal{G}^{\star}(\cdot, \cdot, 0)$ is continuously differentiable imply that the map $k \mapsto \tilde{\Gamma}(\cdot, 0)$ is also continuously differentiable. The continuous differentiability of $k \mapsto \tilde{\Gamma}^{(j)}(\cdot)$, $j=1,2,3$, follows from that of $k \mapsto \mathcal{G}_{1}(\cdot, \cdot, \cdot, 0)$ and equations $(42)-(44)$, while the continuous differentiability of $k \mapsto \tilde{\Gamma}^{\mathrm{r}}(\cdot)$ is a consequence of the formula $\Gamma^{\mathrm{r}}(\eta)=\tilde{\Gamma}(\eta, 0)-\tilde{\Gamma}^{(1)}(\eta)-$ $\tilde{\Gamma}^{(2)}(\eta)-\tilde{\Gamma}^{(3)}(\eta)$.

Corollary 4.12 The linear mappings $\tilde{\mathcal{N}}(\cdot, 0), \tilde{\mathcal{N}}^{(2)}, \tilde{\mathcal{N}}^{(3)}$ and $\tilde{\mathcal{N}}^{\mathrm{r}}: H^{2}(\mathbb{R}) \rightarrow L^{2}(\mathbb{R})$ are continuously differentiable.

Lemma 4.13 The linear mappings $\check{\eta}_{2}(\cdot, 0), \check{\eta}_{2}^{(2)}$ and $\check{\eta}_{2}^{\mathrm{r}}: \chi(D) L^{2}(\mathbb{R}) \rightarrow L^{2}(\mathbb{R})$ are continuously differentiable functions of $k>0$.

Proof. Recall that

$$
\check{\eta}_{2}\left(\eta_{1}, 0\right)=\left(g_{\varepsilon}(D, \varepsilon k)-(1-\chi(D)) \tilde{\mathcal{N}}(\cdot)\right)^{-1}(1-\chi(D)) \tilde{\mathcal{N}}\left(\eta_{1}, 0\right) .
$$

The map $k \mapsto g_{\varepsilon}(D, \varepsilon k)-(1-\chi(D)) \tilde{\mathcal{N}}(\cdot):(1-\chi(D)) H^{2}(\mathbb{R}) \rightarrow(1-\chi(D)) L^{2}(\mathbb{R})$ is continuously differentiable and invertible (Lemma 3.5). It follows that its inverse and hence $\check{\eta}_{2}\left(\eta_{1}\right)$ is continuously differentiable with respect to $k$. The continuous differentiability of $k \mapsto$ $\check{\eta}_{2}^{(2)}(\cdot)$ follows from that of $k \mapsto g_{\varepsilon}(D, \varepsilon k)$, and the continuous differentiability of $k \mapsto \check{\eta}_{2}^{\mathrm{r}}(\cdot)$ is a consequence of the formula $\check{\eta}_{2}^{\mathrm{r}}(\cdot)\left(\eta_{1}\right)=\check{\eta}_{2}\left(\eta_{1}, 0\right)-\check{\eta}_{2}^{(2)}\left(\eta_{1}\right)$.

\section{Corollary 4.14}

(i) The linear mappings $\check{\Gamma}^{(j)}, j=1,2,3$ and $\check{\Gamma}^{\mathrm{r}}: \chi(D) L^{2}(\mathbb{R}) \rightarrow H^{2}(\Sigma)$ are continuously differentiable functions of $k>0$.

(ii) The linear mappings $\check{\mathcal{N}}^{(2)}, \check{\mathcal{N}}^{(3)}$ and $\check{\mathcal{N}}^{\mathrm{r}}: \chi(D) L^{2}(\mathbb{R}) \rightarrow L^{2}(\mathbb{R})$ are continuously differentiable functions of $k>0$.

Corollary 4.15 The linear mappings $\tilde{\mathcal{R}}_{\varepsilon, k}, \mathcal{R}_{\varepsilon, k}: H^{2}(\mathbb{R}) \rightarrow L^{2}(\mathbb{R})$ are continuously differentiable functions of $k>0$.

Having established the continuous differentiability of $\tilde{\mathcal{R}}_{\varepsilon, k}(\zeta)$ and $\mathcal{R}_{\varepsilon, k}(\zeta)$, we now turn to the task of estimating their derivatives, again by systematically examining each step in their derivation. The following result, which is established in the same way as Proposition 3.1, is used in the first step. 
Proposition 4.16 The mapping $\left(P_{1}, P_{2}, P_{3}, P_{4}, P_{5}\right) \mapsto \mathcal{H}\left(P_{1}, P_{2}, P_{3}, P_{4}, P_{5}\right)$, where

$$
\begin{aligned}
& \mathcal{H}\left(P_{1}, P_{2}, P_{3}, P_{4}, P_{5}\right) \\
& \quad=\mathcal{F}^{-1}\left[\int_{0}^{1}\left\{G(y, \tilde{y})\left(-2 \varepsilon^{2} k \hat{P}_{1}-\mathrm{i} \mu \hat{P}_{2}-\mathrm{i} \varepsilon \hat{P}_{3}-\mathrm{i} k \varepsilon \hat{P}_{4}\right)+G_{\tilde{y}}(y, \tilde{y}) \hat{P}_{5}\right\} \mathrm{d} \tilde{y}\right]
\end{aligned}
$$

defines a linear function $H^{1}(\Sigma) \times H^{1}(\Sigma) \times H^{1}(\Sigma) \times H^{1}(\Sigma) \times H^{1}(\Sigma) \rightarrow H^{2}(\Sigma)$ which satisfies the estimate

$$
\|\nabla \mathcal{H}\|_{1}+\varepsilon\|\mathcal{H}\|_{1}+\varepsilon^{2}\|\mathcal{H}\|_{0} \leq c\left(\varepsilon\left\|P_{1}\right\|_{1}+\sum_{j=2}^{5}\left\|P_{j}\right\|_{1}+\varepsilon^{2}\left\|P_{1}\right\|_{0}+\varepsilon \sum_{j=2}^{5}\left\|P_{j}\right\|_{0}\right)
$$

Lemma 4.17 The linear mappings $\tilde{\Gamma}(\cdot, 0), \tilde{\Gamma}^{(j)}, j=1,2,3$, and $\tilde{\Gamma}^{\mathrm{r}}: H^{2}(\mathbb{R}) \rightarrow H^{2}(\Sigma)$ satisfy the estimates

$$
\begin{aligned}
& \left\|\nabla \tilde{\Gamma}(\eta, 0)^{\prime}\right\|_{1}+\varepsilon\left\|\tilde{\Gamma}(\eta, 0)^{\prime}\right\|_{0} \leq c\|\eta\|_{2}, \\
& \left\|\nabla \tilde{\Gamma}^{(j)}(\eta)^{\prime}\right\|_{1}+\varepsilon\left\|\tilde{\Gamma}^{(j)}(\eta)^{\prime}\right\|_{0} \leq c \varepsilon^{j-1}\|\eta\|_{2}, \quad j=1,2,3 \\
& \left\|\nabla \tilde{\Gamma}^{\mathrm{r}}(\eta)^{\prime}\right\|_{1}+\varepsilon\left\|\tilde{\Gamma}^{\mathrm{r}}(\eta)^{\prime}\right\|_{0} \leq c \varepsilon^{3}\|\eta\|_{2}
\end{aligned}
$$

uniformly over $k \in\left[k_{\min }, k_{\max }\right]$.

Proof. Differentiating the boundary-value problem $(33)-(35)$ with respect to $k$, we find that

$$
\begin{aligned}
-\hat{\Gamma}_{y y}^{\prime}+q^{2} \hat{\Gamma}^{\prime} & =-2 \varepsilon^{2} k \hat{\Gamma}-\mathrm{i} \mu \hat{F}_{1}(\eta, \Gamma)^{\prime}-\mathrm{i} \varepsilon F_{2}(\eta, \Gamma)-\mathrm{i} k \varepsilon \hat{F}_{2}(\eta, \Gamma)^{\prime}-\left(\hat{F}_{3}(\eta, \Gamma)\right)_{y}^{\prime}, & & 0<y<1, \\
\hat{\Gamma}_{y}^{\prime} & =0 & & \text { on } y=0, \\
\hat{\Gamma}_{y}^{\prime} & =\hat{F}_{3}(\eta, \Gamma)^{\prime} & & \text { on } y=1,
\end{aligned}
$$

where $\Gamma^{\prime}$ is an abbreviation for $\tilde{\Gamma}(\eta, 0)^{\prime}$ and $F_{j}(\eta, \Gamma)^{\prime}$ is also obtained from $F_{j}(\eta, \Gamma), j=1,2,3$, by differentiating with respect to $k$. It follows that

$$
\tilde{\Gamma}(\eta, 0)^{\prime}=\mathcal{H}\left(\tilde{\Gamma}(\eta, 0), F_{1}(\eta, \tilde{\Gamma}(\eta, 0))^{\prime}, F_{2}(\eta, \tilde{\Gamma}(\eta, 0)), F_{2}(\eta, \tilde{\Gamma}(\eta, 0))^{\prime}, F_{3}(\eta, \tilde{\Gamma}(\eta, 0))^{\prime}\right)
$$

and similarly

$$
\begin{aligned}
\tilde{\Gamma}^{(1)}(\eta)^{\prime} & =\mathcal{H}\left(\tilde{\Gamma}^{(1)}(\eta), 0,0,0,0\right), \\
\tilde{\Gamma}^{(2)}(\eta)^{\prime} & =\mathcal{H}\left(\tilde{\Gamma}^{(2)}(\eta), F_{1}^{(2)}(\eta)^{\prime}, F_{2}^{(2)}(\eta), F_{2}^{(2)}(\eta)^{\prime}, F_{3}^{(2)}(\eta)^{\prime}\right), \\
\tilde{\Gamma}^{(3)}(\eta)^{\prime} & =\mathcal{H}\left(\tilde{\Gamma}^{(3)}(\eta), F_{1}^{(3)}(\eta)^{\prime}, F_{2}^{(3)}(\eta), F_{2}^{(3)}(\eta)^{\prime}, F_{3}^{(3)}(\eta)^{\prime}\right), \\
\tilde{\Gamma}^{\mathrm{r}}(\eta)^{\prime} & =\mathcal{H}\left(\tilde{\Gamma}^{\mathrm{r}}(\eta), F_{1}^{\mathrm{r}}(\eta)^{\prime}, F_{2}^{\mathrm{r}}(\eta), F_{2}^{\mathrm{r}}(\eta)^{\prime}, F_{3}^{\mathrm{r}}(\eta)^{\prime}\right),
\end{aligned}
$$

where $F_{j}^{(2)}(\eta)^{\prime}, F_{j}^{(3)}(\eta)^{\prime}, F_{j}^{\mathrm{r}}(\eta)^{\prime}$ are obtained from $F_{j}^{(2)}(\eta), F_{j}^{(3)}(\eta), F_{j}^{\mathrm{r}}(\eta), j=1,2,3$, by differentiating with respect to $k$. The estimates stated in the lemma are obtained by applying Proposition 4.16 to these formulae (estimates for $\tilde{\Gamma}(\eta, 0)$ and $\tilde{\Gamma}^{(j)}(\eta), \tilde{\Gamma}^{\mathrm{r}}(\eta)$ are given in respectively Theorem 3.2 and Lemma 3.7. 
Corollary 4.18 The linear mappings $\tilde{\mathcal{N}}(\cdot, 0), \tilde{\mathcal{N}}^{(2)}, \tilde{\mathcal{N}}^{(3)}$ and $\tilde{\mathcal{N}}^{\mathrm{r}}: H^{2}(\mathbb{R}) \rightarrow L^{2}(\mathbb{R})$ satisfy the estimates

$$
\begin{aligned}
\left\|\tilde{\mathcal{N}}(\eta, 0)^{\prime}\right\|_{0} & \leq c \varepsilon\|\eta\|_{2}, \\
\left\|\tilde{\mathcal{N}}^{(j)}(\eta)^{\prime}\right\|_{0} & \leq c \varepsilon^{j-1}\|\eta\|_{2}, \quad j=2,3 \\
\left\|\tilde{\mathcal{N}}^{\mathrm{r}}(\eta)^{\prime}\right\|_{0} & \leq c \varepsilon^{3}\|\eta\|_{2}
\end{aligned}
$$

uniformly over $k \in\left[k_{\min }, k_{\max }\right]$.

The next estimates are obtained by differentiating the formulae defining $\check{\eta}_{2}\left(\eta_{1}, 0\right), \check{\eta}_{2}^{(2)}\left(\eta_{1}\right)$ and $\check{\eta}_{2}^{\mathrm{r}}\left(\eta_{1}\right)$ with respect to $k$ and using Lemma 3.8. Proposition 3.9(i), Corollary 4.18 and the fact that $\left|\partial_{k} g_{\varepsilon}(\mu, \varepsilon k)\right| \leq c$ for $k \in\left[k_{\min }, k_{\max }\right]$.

Lemma 4.19 The linear mappings $\check{\eta}_{2}(\cdot, 0), \check{\eta}_{2}^{(2)}$ and $\check{\eta}_{2}^{\mathrm{r}}: \chi(D) L^{2}(\mathbb{R}) \rightarrow L^{2}(\mathbb{R})$ satisfy the estimates

$$
\left\|\left(\check{\eta}_{2}\left(\eta_{1}, 0\right)\right)^{\prime}\right\|_{2} \leq c \varepsilon\left\|\eta_{1}\right\|_{0}, \quad\left\|\left(\check{\eta}_{2}^{(2)}\left(\eta_{1}\right)\right)^{\prime}\right\|_{2} \leq c \varepsilon\left\|\eta_{1}\right\|_{0}, \quad\left\|\left(\check{\eta}_{2}^{\mathrm{r}}\left(\eta_{1}\right)\right)^{\prime}\right\|_{2} \leq c \varepsilon^{2}\left\|\eta_{1}\right\|_{0}
$$

uniformly over $k \in\left[k_{\min }, k_{\max }\right]$.

\section{Corollary 4.20}

(i) The linear mappings $\check{\Gamma}^{(j)}, j=1,2,3$ and $\check{\Gamma}^{\mathrm{r}}: \chi(D) L^{2}(\mathbb{R}) \rightarrow H^{2}(\Sigma)$ satisfy the estimates

$$
\begin{gathered}
\left\|\nabla \check{\Gamma}^{(j)}\left(\eta_{1}\right)^{\prime}\right\|_{1}+\varepsilon\left\|\check{\Gamma}^{(j)}\left(\eta_{1}\right)^{\prime}\right\|_{0} \leq c \varepsilon^{j-1}\left\|\eta_{1}\right\|_{0}, \quad j=1,2,3 \\
\left\|\nabla \check{\Gamma}^{\mathrm{r}}\left(\eta_{1}\right)^{\prime}\right\|_{1}+\varepsilon\left\|\check{\Gamma}^{\mathrm{r}}\left(\eta_{1}\right)^{\prime}\right\|_{0} \leq c \varepsilon^{3}\left\|\eta_{1}\right\|_{0}
\end{gathered}
$$

uniformly over $k \in\left[k_{\min }, k_{\max }\right]$.

(ii) The linear mappings $\check{\mathcal{N}}^{(2)}, \check{\mathcal{N}}^{(3)}$ and $\check{\mathcal{N}}^{\mathrm{r}}: \chi(D) L^{2}(\mathbb{R}) \rightarrow L^{2}(\mathbb{R})$ satisfy the estimates

$$
\begin{aligned}
\left\|\check{\mathcal{N}}^{(j)}\left(\eta_{1}\right)^{\prime}\right\|_{0} & \leq c \varepsilon^{j-1}\left\|\eta_{1}\right\|_{0}, \quad j=2,3 \\
\left\|\check{\mathcal{N}}^{\mathrm{r}}\left(\eta_{1}\right)^{\prime}\right\|_{0} & \leq c \varepsilon^{3}\left\|\eta_{1}\right\|_{0}
\end{aligned}
$$

uniformly over $k \in\left[k_{\min }, k_{\max }\right]$.

An estimate for $\check{\mathcal{N}}^{(2)}\left(\eta_{1}\right)^{\prime}$ as a function of $\zeta$ is given in Lemma 4.22. This result is established by differentiating (49) and using Propositions 3.14, 3.16 and 4.21 below, which is derived from the formulae

$$
\check{\Gamma}^{(1) \prime}=\mathcal{F}^{-1}\left[\int_{0}^{1} G(y, \tilde{y})\left(-2 \varepsilon^{2} k \mathcal{F}\left[\check{\Gamma}_{1}\right]\right) \mathrm{d} \tilde{y}\right]
$$

and

$$
\begin{gathered}
\tilde{\Gamma}^{(2)}\left(\eta_{1}\right)^{\prime}=\mathcal{F}^{-1}\left[\int _ { 0 } ^ { 1 } \left\{G(y, \tilde{y})\left(-2 \varepsilon^{2} k \mathcal{F}\left[\tilde{\Gamma}^{(2)}\left(\eta_{1}\right)\right]-\mathrm{i} \mu \mathcal{F}\left[\check{F}_{1}^{(2) \prime}\right]-\mathrm{i} \varepsilon \mathcal{F}\left[\check{F}_{2}^{(2)}\right]-\mathrm{i} k \varepsilon \mathcal{F}\left[\check{F}_{2}^{(2) \prime}\right]\right)\right.\right. \\
\left.\left.+G_{\tilde{y}}(y, \tilde{y}) \mathcal{F}\left[F_{3}^{(2) \prime}\right]\right\} \mathrm{d} \tilde{y}\right]
\end{gathered}
$$

by the methods used in Section 3.3 . 
Proposition 4.21 The quantities $\check{\Gamma}^{(1) \prime}$ and $\tilde{\Gamma}^{(2)}\left(\eta_{1}\right)^{\prime}$ satisfy the estimates

$$
\begin{aligned}
\check{\Gamma}^{(1) \prime} & =S, \\
\tilde{\Gamma}^{(2)}\left(\eta_{1}\right)^{\prime} & =-2 \varepsilon \mathcal{F}^{-1}\left[\frac{\mathrm{i} \mu k}{\left(\mu^{2}+k^{2}\right)^{2}} \mathcal{F}\left[\operatorname{Re}\left(\zeta^{\star} \zeta\right)\right](\mu)\right](\varepsilon x) \mu_{0} \operatorname{coth} \mu_{0}+\varepsilon S
\end{aligned}
$$

uniformly over $k \in\left[k_{\min }, k_{\max }\right]$.

Lemma 4.22 The quantity $\check{\mathcal{N}}^{(2)}\left(\eta_{1}\right)^{\prime}$ satisfies the estimate

$$
\check{\mathcal{N}}^{(2)}\left(\eta_{1}\right)^{\prime}=2 \varepsilon^{2} \mathcal{F}^{-1}\left[\frac{\mu^{2} k}{\left(\mu^{2}+k^{2}\right)^{2}} \mathcal{F}\left[\operatorname{Re}\left(\zeta_{\delta}^{\star} \zeta\right)\right](\mu)\right](\varepsilon x) \frac{\mu_{0} \cosh \left(\mu_{0}\right)}{\sinh \left(\mu_{0}\right)}+\varepsilon R_{0}
$$

uniformly over $k \in\left[k_{\min }, k_{\max }\right]$.

Lemma 4.23 The quantity $\check{\eta}_{2}^{(2) \prime}$ satisfies the estimate

$$
\check{\eta}_{2}^{(2) \prime}=2 \varepsilon^{2} B_{3} \mathcal{F}^{-1}\left[\frac{\mu^{2} k}{\left(\left(1-\alpha_{0}^{-1}\right) \mu^{2}+k^{2}\right)^{2}} \mathcal{F}\left[2 \operatorname{Re}\left(\zeta_{\delta}^{\star} \zeta\right)\right](\mu)\right](\varepsilon x)+\varepsilon R_{2}
$$

uniformly over $k \in\left[k_{\min }, k_{\max }\right]$.

Proof. Differentiating the formula

$$
g_{\varepsilon}(\mu, \varepsilon k) \mathcal{F}\left[\check{\eta}^{(2)}\right]=(1-\chi) \mathcal{F}\left[\check{\mathcal{N}}^{(2)}\left(\eta_{1}\right)\right]=\mathcal{F}\left[\check{\mathcal{N}}^{(2)}\left(\eta_{1}\right)\right]
$$

with respect to $k$ yields

$$
\begin{aligned}
& g(\mu, \varepsilon k) \mathcal{F}\left[\check{\eta}_{2}^{(2) '}\right]=\mathcal{F}\left[\check{\mathcal{N}}^{(2)}\left(\eta_{1}\right)^{\prime}\right]-\partial_{k} g_{\varepsilon}(\mu, \varepsilon k) \mathcal{F}\left[\check{\eta}^{(2)}\left(\eta_{1}\right)\right] \\
&=2 \varepsilon \frac{\left(\varepsilon^{-1} \mu\right)^{2} k}{\left(\left(\varepsilon^{-1} \mu\right)^{2}+k^{2}\right)^{2}} \mathcal{F}\left[\operatorname{Re}\left(\zeta_{\delta}^{\star} \zeta\right)\right]\left(\varepsilon^{-1} \mu\right) \frac{\mu_{0} \cosh \left(\mu_{0}\right)}{\sinh \left(\mu_{0}\right)} \\
&+\varepsilon \partial_{k} g_{\varepsilon}(\mu, \varepsilon k)\left(\frac{1}{2} B_{1} \mathcal{F}\left[\zeta_{\delta}^{\star} \zeta\right]\left(\varepsilon^{-1}\left(\mu-2 \mu_{0}\right)\right)+\frac{1}{2} B_{1} \mathcal{F}\left[\zeta_{\delta}^{\star} \bar{\zeta}\right]\left(\varepsilon^{-1}\left(\mu+2 \mu_{0}\right)\right)\right) \\
&+\varepsilon \partial_{k} g_{\varepsilon}(\mu, \varepsilon k)\left(B_{2}+B_{3} \frac{2\left(\varepsilon^{-1} \mu\right)^{2}}{\left(1-\alpha_{0}^{-1}\right)\left(\varepsilon^{-1} \mu\right)^{2}+k^{2}}\right) \mathcal{F}\left[\operatorname{Re}\left(\zeta_{\delta}^{\star} \zeta\right)\right]\left(\varepsilon^{-1} \mu\right) \\
&+\varepsilon(1-\chi) \hat{R}_{0},
\end{aligned}
$$

in which we have used Proposition 3.18 and Lemma 4.22. Noting that

$$
\partial_{k} g_{\varepsilon}(\mu, \varepsilon k)=\frac{2 k\left(\varepsilon^{-1} \mu\right)^{2}}{\left(\left(\varepsilon^{-1} \mu\right)^{2}+k^{2}\right)^{2}}+O(\varepsilon)
$$

uniformly over $\left\{|\mu| \in\left[0, \mu_{\max }\right]\right\}$ for each fixed $\mu_{\max }>0$ (in particular for $\mu_{\max }=2 \delta$ ), whence $\partial_{k} g_{\varepsilon}(\mu, \varepsilon k)=O(\varepsilon)$ uniformly over $\left\{|\mu| \in\left[\mu_{\min }, \mu_{\max }\right]\right\}$ for each fixed $\mu_{\min }, \mu_{\max }>0$ (in particular for $\left.\mu_{\min }=2 \mu_{0}-2 \delta, \mu_{\max }=2 \mu_{0}+2 \delta\right)$, we find that

$$
\begin{aligned}
& g(\mu, \varepsilon k) \mathcal{F}\left[\check{\eta}_{2}^{(2)^{\prime}}\right] \\
& =2 \varepsilon \frac{\left(\varepsilon^{-1} \mu\right)^{2} k}{\left(\left(\varepsilon^{-1} \mu\right)^{2}+k^{2}\right)^{2}}\left(\frac{\mu_{0} \cosh \left(\mu_{0}\right)}{\sinh \left(\mu_{0}\right)}+B_{2}+B_{3} \frac{2\left(\varepsilon^{-1} \mu\right)^{2}}{\left(\alpha_{0}-1\right)\left(\varepsilon^{-1} \mu\right)^{2}+\alpha_{0} k^{2}}\right) \mathcal{F}\left[\operatorname{Re}\left(\zeta_{\delta}^{\star} \zeta\right)\right]\left(\varepsilon^{-1} \mu\right) \\
& \quad+\varepsilon(1-\chi) \hat{R}_{0} .
\end{aligned}
$$


Finally, we use the estimate

$$
g_{\varepsilon}(\mu, k \varepsilon)^{-1}=\frac{\left(\varepsilon^{-1} \mu\right)^{2}+k^{2}}{\left(\alpha_{0}-1\right)\left(\varepsilon^{-1} \mu\right)^{2}+\alpha_{0} k^{2}}+T_{2}(\mu, \varepsilon),
$$

where

$$
\left|T_{2}(\mu, \varepsilon)\right| \leq c\left(\mu^{2}+\varepsilon^{2}\right), \quad|\mu|<2 \delta,
$$

and the fact that

$$
g_{\varepsilon}(D, \lambda)^{-1}(1-\chi(D)) R_{0}=R_{2}
$$

because $g_{\varepsilon}(\mu, k \varepsilon)>c\left(1+q^{2}\right)$ for $\mu \in \operatorname{supp}(1-\chi)$ (see Proposition 3.3.

Finally, we compute estimates for $\check{\Gamma}^{(2) \prime}$ and $\tilde{\Gamma}^{(3)}\left(\eta_{1}\right)^{\prime}+\tilde{\Gamma}^{(2)}\left(\check{\eta}_{2}^{(2)}\right)^{\prime}$ using the formulae

$$
\check{\Gamma}^{(2) \prime}=\tilde{\Gamma}^{(2)}\left(\eta_{1}\right)^{\prime}+\tilde{\Gamma}^{(1)}\left(\check{\eta}_{2}^{(2)}\right)^{\prime}, \quad \tilde{\Gamma}^{(1)}\left(\check{\eta}_{2}^{(2)}\right)^{\prime}=\mathcal{F}^{-1}\left[\int_{0}^{1} G(y, \tilde{y})\left(-2 \varepsilon^{2} k \mathcal{F}\left[\tilde{\Gamma}^{(1)}\left(\check{\eta}_{2}^{(2)}\right)\right]\right) \mathrm{d} \tilde{y}\right]
$$

and

$$
\begin{aligned}
& \tilde{\Gamma}^{(3)}\left(\eta_{1}\right)^{\prime}+\tilde{\Gamma}^{(2)}\left(\check{\eta}_{2}^{(2)}\right)^{\prime} \\
& =\mathcal{F}^{-1}\left[\int _ { 0 } ^ { 1 } \left\{G(y, \tilde{y})\left(-2 \varepsilon^{2} k \mathcal{F}\left[\tilde{\Gamma}^{(3)}\left(\eta_{1}\right)+\tilde{\Gamma}^{(2)}\left(\check{\eta}_{2}^{(2)}\right)\right]-\mathrm{i} \mu \mathcal{F}\left[\check{F}_{1}^{(3) \prime}\right]-\mathrm{i} \varepsilon \mathcal{F}\left[\check{F}_{2}^{(3)}\right]-\mathrm{i} k \varepsilon \mathcal{F}\left[\check{F}_{2}^{(3) \prime}\right]\right)\right.\right. \\
& \left.\left.\quad+G_{\tilde{y}}(y, \tilde{y}) \mathcal{F}\left[F_{3}^{(3) \prime}\right]\right\} \mathrm{d} \tilde{y}\right]
\end{aligned}
$$

and deduce an estimate for $\check{\mathcal{N}}\left(\eta_{1}\right)^{\prime}$ by differentiating equation 55 .

\section{Proposition 4.24}

(i) The quantity $\check{\Gamma}^{(2)}\left(\eta_{1}\right)^{\prime}$ satisfies the estimate

$$
\check{\Gamma}^{(2)}\left(\eta_{1}\right)^{\prime}=-\varepsilon\left(\vartheta_{\delta}(\varepsilon x)\right)^{\prime} M_{2}+\varepsilon S
$$

uniformly over $k \in\left[k_{\min }, k_{\max }\right]$, where

$$
\left(\vartheta_{\delta}(x)\right)^{\prime}=-2 A_{4} \mathcal{F}^{-1}\left[\frac{\mathrm{i} \mu k}{\left(\left(1-\alpha_{0}^{-1}\right) \mu^{2}+k^{2}\right)^{2}} \mathcal{F}\left[2 \operatorname{Re}\left(\zeta_{\delta}^{\star} \zeta\right)\right](\mu)\right](x) .
$$

(ii) The quantity $\tilde{\Gamma}_{x}^{(3)}\left(\eta_{1}\right)^{\prime}+\left.\tilde{\Gamma}_{x}^{(2)}\left(\check{\eta}_{2}^{(2)}\right)^{\prime}\right|_{y=1}$ satisfies the estimate

$$
\tilde{\Gamma}_{x}^{(3)}\left(\eta_{1}\right)^{\prime}+\left.\tilde{\Gamma}_{x}^{(2)}\left(\check{\eta}_{2}^{(2)}\right)^{\prime}\right|_{y=1}=\mu_{0} \varepsilon^{3} \zeta_{\delta}^{\star}(\varepsilon x) \vartheta_{\delta x}(\varepsilon x)^{\prime} N_{3}\left(\frac{1}{2} \mathrm{e}^{\mathrm{i} \mu_{0} x}+\frac{1}{2} \mathrm{e}^{-\mathrm{i} \mu_{0} x}\right)+\varepsilon^{2} T
$$

uniformly over $k \in\left[k_{\min }, k_{\max }\right]$.

Lemma 4.25 The quantity $\check{\mathcal{N}}^{(3)}\left(\eta_{1}\right)^{\prime}$ is given by the formula

$$
\check{\mathcal{N}}^{(3)}\left(\eta_{1}\right)^{\prime}=-4 \varepsilon^{3} A_{4} \zeta_{\delta}^{\star}(\varepsilon x) \vartheta_{\delta x}(\varepsilon x)^{\prime}\left(\frac{1}{2} \mathrm{e}^{\mathrm{i} \mu_{0} x}+\frac{1}{2} \mathrm{e}^{-\mathrm{i} \mu_{0} x}\right)+\varepsilon^{2} R
$$

uniformly over $k \in\left[k_{\min }, k_{\max }\right]$. 
We now prove our main result using Lemmata 3.17, 3.22, 4.22 and 4.25 .

Theorem 4.26 The linear mappings $\tilde{\mathcal{R}}_{\varepsilon, k}, \mathcal{R}_{\varepsilon, k}: H^{2}(\mathbb{R}) \rightarrow L^{2}(\mathbb{R})$ satisfy the estimate

$$
\left\|\tilde{\mathcal{R}}_{\varepsilon, k}(\zeta)^{\prime}\right\|_{0},\left\|\mathcal{R}_{\varepsilon, k}(\zeta)^{\prime}\right\|_{0} \leq c \varepsilon\|\zeta\|_{1}
$$

uniformly over $k \in\left[k_{\min }, k_{\max }\right]$.

Proof. Recall that

$$
\begin{aligned}
\tilde{\mathcal{R}}_{\varepsilon, k}(\zeta)= & A_{2}^{-1} \mathcal{F}^{-1}\left[2 \frac{\tilde{g}_{\varepsilon}\left(\mu_{0}+\varepsilon \tilde{\mu}, \varepsilon k\right)}{g_{\varepsilon}\left(\mu_{0}+\varepsilon \tilde{\mu}, \varepsilon k\right)} \chi_{0}(\varepsilon \tilde{\mu}) \varepsilon^{-2} \mathcal{F}\left[\check{\mathcal{N}}^{(3)}\left(\chi(D) \eta_{1}\right)+\check{\mathcal{N}}^{\mathrm{r}}\left(\chi(D) \eta_{1}\right)\right]\left(\mu_{0}+\varepsilon \tilde{\mu}\right)\right] \\
& -A_{2}^{-1} E(x)
\end{aligned}
$$

so that

$$
\begin{aligned}
\tilde{\mathcal{R}}_{\varepsilon, k}(\zeta)^{\prime}= & A_{2}^{-1} \mathcal{F}^{-1}\left[2\left(\frac{\tilde{g}_{\varepsilon}\left(\mu_{0}+\varepsilon \tilde{\mu}, \varepsilon k\right)}{g_{\varepsilon}\left(\mu_{0}+\varepsilon \tilde{\mu}, \varepsilon k\right)}\right)^{\prime} \chi_{0}(\varepsilon \tilde{\mu}) \varepsilon^{-2} \mathcal{F}\left[\check{\mathcal{N}}^{(3)}\left(\chi(D) \eta_{1}\right)+\check{\mathcal{N}}^{\mathrm{r}}\left(\chi(D) \eta_{1}\right)\right]\left(\mu_{0}+\varepsilon \tilde{\mu}\right)\right] \\
& +A_{2}^{-1} \mathcal{F}^{-1}\left[2 \frac{\tilde{g}_{\varepsilon}\left(\mu_{0}+\varepsilon \tilde{\mu}, \varepsilon k\right)}{g_{\varepsilon}\left(\mu_{0}+\varepsilon \tilde{\mu}, \varepsilon k\right)} \chi_{0}(\varepsilon \tilde{\mu}) \varepsilon^{-2} \mathcal{F}\left[\check{\mathcal{N}}^{(3)}\left(\chi(D) \eta_{1}\right)^{\prime}+\check{\mathcal{N}}^{\mathrm{r}}\left(\chi(D) \eta_{1}\right)^{\prime}\right]\left(\mu_{0}+\varepsilon \tilde{\mu}\right)\right] \\
& -A_{2}^{-1} E(x)^{\prime} .
\end{aligned}
$$

Noting that

$$
\check{\mathcal{N}}^{(3)}\left(\chi(D) \eta_{1}\right)^{\prime}=\frac{\varepsilon^{3}}{2} E(\varepsilon x)^{\prime} \mathrm{e}^{\mathrm{i} \mu_{0} x}+\frac{\varepsilon^{3}}{2} F(\varepsilon x)^{\prime} \mathrm{e}^{-\mathrm{i} \mu_{0} x}+\varepsilon^{2} T,
$$

one finds by repeating the arguments below equation $(60)$ that

$$
\begin{aligned}
& \left\|\mathcal{F}^{-1}\left[2 \frac{\tilde{g}_{\varepsilon}\left(\mu_{0}+\varepsilon \tilde{\mu}, \varepsilon k\right)}{g_{\varepsilon}\left(\mu_{0}+\varepsilon \tilde{\mu}, \varepsilon k\right)} \chi_{0}(\varepsilon \tilde{\mu}) \varepsilon^{-2} \mathcal{F}\left[\check{\mathcal{N}}^{(3)}\left(\chi(D) \eta_{1}\right)^{\prime}+\check{\mathcal{N}}^{\mathrm{r}}\left(\chi(D) \eta_{1}\right)^{\prime}\right]\left(\mu_{0}+\varepsilon \tilde{\mu}\right)\right]-A_{2}^{-1} E(x)^{\prime}\right\|_{0} \\
& \leq c\|\zeta\|_{1} .
\end{aligned}
$$

Moreover, the calculation

$$
\left(\frac{\tilde{g}_{\varepsilon}(\mu, \varepsilon k)}{g_{\varepsilon}(\mu, \varepsilon k)}\right)^{\prime}=\frac{1}{g_{\varepsilon}(\mu, \varepsilon k)}\left(\left(1-\frac{\tilde{g}_{\varepsilon}(\mu, \varepsilon k)}{g_{\varepsilon}(\mu, \varepsilon k)}\right) \partial_{k} g_{\varepsilon}(\mu, \varepsilon k)+\partial_{k} g_{\varepsilon}(\mu, \varepsilon k)-\partial_{k} \tilde{g}_{\varepsilon}(\mu, \varepsilon k)\right)
$$

and the estimates

$$
\begin{gathered}
c\left|\left(\mu-\mu_{0}, \varepsilon k\right)\right|^{2} \leq g_{\varepsilon}(\mu, \varepsilon k) \leq \frac{1}{c}\left|\left(\mu-\mu_{0}, \varepsilon k\right)\right|^{2}, \quad\left|\frac{\tilde{g}_{\varepsilon}(\mu, \varepsilon k)}{g_{\varepsilon}(\mu, \varepsilon k)}-1\right| \leq c\left|\left(\mu-\mu_{0}, \varepsilon k\right)\right|^{2} \\
\left|\partial_{k} g(\mu, \varepsilon k)\right| \leq c \varepsilon, \quad\left|\partial_{k} g_{\varepsilon}(\mu, \varepsilon k)-\partial_{k} \tilde{g}_{\varepsilon}(\mu, \varepsilon k)\right| \leq c \varepsilon\left|\left(\mu-\mu_{0}, \varepsilon k\right)\right|^{3}
\end{gathered}
$$

for $\left|\mu-\mu_{0}\right|<\delta$ imply that

$$
\left|\left(\frac{\tilde{g}_{\varepsilon}\left(\mu_{0}+\varepsilon \tilde{\mu}, \varepsilon k\right)}{g_{\varepsilon}\left(\mu_{0}+\varepsilon \tilde{\mu}, \varepsilon k\right)}\right)^{\prime} \chi_{0}(\varepsilon \tilde{\mu})\right| \leq c \varepsilon,
$$


whence

$$
\begin{aligned}
& \left\|\mathcal{F}^{-1}\left[2\left(\frac{\tilde{g}_{\varepsilon}\left(\mu_{0}+\varepsilon \tilde{\mu}, \varepsilon k\right)}{g_{\varepsilon}\left(\mu_{0}+\varepsilon \tilde{\mu}, \varepsilon k\right)}\right)^{\prime} \chi_{0}(\varepsilon \tilde{\mu}) \varepsilon^{-2} \mathcal{F}\left[\check{\mathcal{N}}^{(3)}\left(\eta_{1}\right)+\check{\mathcal{N}}^{\mathrm{r}}\left(\eta_{1}\right)\right]\left(\mu_{0}+\varepsilon \tilde{\mu}\right)\right]\right\|_{0} \\
& \leq c \varepsilon^{-3 / 2}\left(\left\|\check{\mathcal{N}}^{(3)}\left(\chi(D) \eta_{1}\right)\right\|_{0}+\left\|\check{\mathcal{N}}^{\mathrm{r}}\left(\chi(D) \eta_{1}\right)\right\|_{0}\right) \\
& \leq c \varepsilon^{1 / 2}\left\|\eta_{1}\right\|_{1} \\
& \leq c \varepsilon\|\zeta\|_{1} .
\end{aligned}
$$

According to the above calculation

$$
\left\|\tilde{\mathcal{R}}_{\varepsilon, k}(\zeta)^{\prime}\right\|_{0} \leq c \varepsilon\|\zeta\|_{1},
$$

and the observation

$$
\mathcal{R}_{\varepsilon, k}(\zeta)^{\prime}-\tilde{\mathcal{R}}_{\varepsilon, k}^{\prime}(\zeta)=A_{4}\left(\zeta_{\delta}^{\star} \vartheta_{\delta x}^{\prime}-\zeta \vartheta_{x}^{\prime}\right)
$$

shows that

$$
\left\|\mathcal{R}_{\varepsilon, k}(\zeta)^{\prime}-\tilde{\mathcal{R}}_{\varepsilon, k}^{\prime}(\zeta)\right\|_{0} \leq c \varepsilon\|\zeta\|_{1}
$$

Corollary 4.27 The linear mapping $\tilde{\mathcal{B}}_{\varepsilon, k}: \mathcal{D}_{\mathcal{B}} \rightarrow V$ satisfies the estimate

$$
\left\|\tilde{\mathcal{B}}_{\varepsilon, k}^{\prime}\right\|_{\mathcal{L}\left(\mathcal{D}_{\mathcal{B}}, V\right)} \leq c \varepsilon
$$

uniformly over $k \in\left[k_{\min }, k_{\max }\right]$.

The results in the next lemma are deduced from the estimates

$$
\left\|\tilde{\mathcal{B}}_{\varepsilon, k}-\tilde{\mathcal{B}}_{0, k}\right\|_{\mathcal{L}\left(\mathcal{D}_{\mathcal{B}}, V\right)} \leq c \varepsilon, \quad\left\|\tilde{\mathcal{B}}_{\varepsilon, k}^{\prime}\right\|_{\mathcal{L}\left(\mathcal{D}_{\mathcal{B}}, V\right)} \leq c \varepsilon
$$

using the method given by Groves, Haragus \& Sun [14, Lemma 3.15].

Lemma 4.28 The eigenvalue $\kappa_{\varepsilon, k}$ of $\tilde{\mathcal{B}}_{\varepsilon, k}: \mathcal{D}_{\mathcal{B}} \subseteq V \rightarrow V$ is a differentiable function of $k$ and satisfies the estimates

$$
\left|\kappa_{\varepsilon, k}-\kappa_{0, k}\right| \leq c^{\star} \varepsilon, \quad\left|\kappa_{\varepsilon, k}^{\prime}\right| \leq c^{\star} \varepsilon
$$

uniformly in $k \in\left[k_{\min }, k_{\max }\right]$.

Finally, the solution set of $(72)$ in the interval $\left[k_{\min }, k_{\max }\right]$ can be determined from the estimates presented in the previous lemma using a perturbation argument and the contractionmapping principle (cf. Groves, Haragus \& Sun [14, Theorem 3.16]).

Theorem 4.29 Equation (72) has precisely one solution $k_{\varepsilon}$ in the interval $\left[k_{\min }, k_{\max }\right]$. This solution lies in the set

$$
\mathcal{S}=\left\{k:\left|k-k_{0}\right| \leq \frac{c^{\star} \varepsilon}{k_{0}}\right\}
$$




\section{Appendix: The Iooss-Kirchgässner line solitary waves}

An asymptotic expansion of the Iooss-Kirchgässner line solitary waves in powers of $\varepsilon$ may be computed in the framework of their existence theory (see Dias \& Iooss [7], noting the omission of certain terms in equation (3.41)). One finds that

$$
\eta^{\star}(x)=\eta_{1}^{\star}(x)+\eta_{2}^{\star}(x)+\eta_{\mathrm{r}}^{\star}(x), \quad \Phi^{\star}(x, y)=\Phi_{1}^{\star}(x, y)+\Phi_{2}^{\star}(x, y)+\Phi_{\mathrm{r}}^{\star}(x, y),
$$

where

$$
\begin{aligned}
\eta_{1}^{\star}(x)= & \varepsilon \zeta^{\star}(\varepsilon x) \cos \left(\mu_{0} x\right), \\
\eta_{2}^{\star}(x)= & C_{0} \varepsilon^{2} \zeta^{\star}(\varepsilon x) \xi^{\star}(\varepsilon x) \sin \left(\mu_{0} x\right)-C_{1} \varepsilon^{2}\left(\zeta^{\star}\right)^{2}(\varepsilon x) \cos \left(2 \mu_{0} x\right) \\
& \quad-C_{2} \varepsilon^{2}\left(\zeta^{\star}\right)^{2}(\varepsilon x)+C_{3} \varepsilon^{2}\left(\zeta^{\star}\right)^{\prime}(\varepsilon x) \sin \left(\mu_{0} x\right), \\
\Phi_{1}^{\star}(x, y)= & \varepsilon \zeta^{\star}(\varepsilon x) \sin \left(\mu_{0} x\right) \frac{\cosh \left(\mu_{0} y\right)}{\sinh \left(\mu_{0}\right)}, \\
\Phi_{2}^{\star}(x, y)= & -C_{0} \varepsilon^{2} \xi^{\star}(\varepsilon x) \cos \left(\mu_{0} x\right) \frac{\cosh \left(\mu_{0} y\right)}{\sinh \mu_{0}}+\varepsilon^{2}\left(\zeta^{\star}\right)^{2}(\varepsilon x) \sin \left(2 \mu_{0} x\right) \frac{\mu_{0} y \sinh \left(\mu_{0} y\right)}{2 \sinh \left(\mu_{0}\right)} \\
& \quad-C_{4} \varepsilon \partial_{x}^{-1}\left(\zeta^{\star 2}\right)(\varepsilon x)-C_{5} \varepsilon^{2}\left(\zeta^{\star}\right)^{2}(\varepsilon x) \sin \left(2 \mu_{0} x\right) \frac{\cosh \left(2 \mu_{0} y\right)}{\sinh \left(2 \mu_{0}\right)},
\end{aligned}
$$

and $\eta_{\mathrm{r}}^{\star}, \nabla \Gamma_{\mathrm{r}}^{\star}=\underline{O}\left(\varepsilon^{3}\right)$, the symbol $\underline{O}\left(\varepsilon^{\alpha}\right)$ denoting a smooth quantity whose derivatives are all $O\left(\varepsilon^{\alpha} \mathrm{e}^{-\rho \varepsilon|x|}\right)$ for some $\rho>0$ (uniformly over $y \in[0,1]$ ). The functions $\zeta^{\star}$ and $\xi^{\star}$ are given by the formulae

$$
\zeta^{\star}(x)=\left(\frac{2}{A_{5}}\right)^{1 / 2} \operatorname{sech}\left(\frac{x}{A_{1}^{1 / 2}}\right), \quad \xi^{\star}(x)=x \zeta^{\star}(x)
$$

with positive constants

$$
A_{1}=\beta_{0}+\left(1-\mu_{0} \operatorname{coth}\left(\mu_{0}\right)\right) \operatorname{cosech}^{2}\left(\mu_{0}\right), \quad A_{5}=A_{3}+4\left(1-\alpha_{0}^{-1}\right)^{-1} A_{4}^{2},
$$

where

$$
\begin{aligned}
& A_{3}=-\frac{\mu_{0}^{3}}{8 \sigma^{3}}\left(\frac{\left(1-\sigma^{2}\right)\left(9-\sigma^{2}\right) \alpha_{0}+\beta_{0} \mu_{0}^{2}\left(3-\sigma^{2}\right)\left(7-\sigma^{2}\right)}{\alpha_{0} \sigma^{2}-\beta_{0} \mu_{0}^{2}\left(3-\sigma^{2}\right)}+8 \sigma^{2}-\frac{2 \mu_{0}}{\alpha_{0} \sigma}\left(1-\sigma^{2}\right)^{2}-3 \beta_{0} \mu_{0} \sigma^{3}\right), \\
& A_{4}=\frac{\mu_{0}\left(\alpha_{0} \sinh \left(2 \mu_{0}\right)+\mu_{0}\right)}{4 \alpha_{0} \sinh ^{2}\left(\mu_{0}\right)}
\end{aligned}
$$

and

$$
\sigma=\tanh \left(\mu_{0}\right)=\frac{\mu_{0}}{\alpha_{0}+\beta_{0} \mu_{0}^{2}}
$$

Finally, the positive coefficients $C_{1}, C_{2}, C_{4}$ and $C_{5}$ are given by

$$
\begin{aligned}
C_{1} & =\frac{\mu_{0}^{2}\left(\cosh \left(2 \mu_{0}\right)+2\right)}{4 \sinh ^{2}\left(\mu_{0}\right) g_{0}\left(2 \mu_{0}, 0\right)}, & C_{2} & =\frac{\mu_{0}\left(\sinh \left(2 \mu_{0}\right)+\mu_{0}\right)}{4 \sinh ^{2}\left(\mu_{0}\right)\left(\alpha_{0}-1\right)}, \\
C_{4} & =\frac{\mu_{0}\left(\alpha_{0} \sinh \left(2 \mu_{0}\right)+\mu_{0}\right)}{4 \sinh ^{2}\left(\mu_{0}\right)\left(\alpha_{0}-1\right)}, & C_{5} & =\frac{\mu_{0}^{2}\left(\cosh \left(2 \mu_{0}\right)+2\right)}{4 \sinh ^{2}\left(\mu_{0}\right) g_{0}\left(2 \mu_{0}, 0\right)}+\frac{\mu_{0} \sinh \left(2 \mu_{0}\right)}{4 \sinh ^{2}\left(\mu_{0}\right)},
\end{aligned}
$$

while the values (and signs) of the coefficients $C_{0}$ and $C_{3}$ are unimportant.

Acknowledgements. E. Wahlén was supported by the Swedish Research Council (grant no. 6212012-3753). We would like to thank Mariana Haragus (Université de Franche-Comté Besançon) for many helpful discussions during the preparation of this article. 


\section{References}

[1] Ablowitz, M. J. \& Segur, H. 1979 On the evolution of packets of water waves. J. Fluid Mech. 92, 691-715.

[2] AMICK, C. J. \& KIRCHGÄSSNER, K. 1989 A theory of solitary water waves in the presence of surface tension. Arch. Rat. Mech. Anal. 105, 1-49.

[3] BAgri, G. \& Groves, M. D. 2014 A spatial dynamics theory for doubly periodic travelling gravity-capillary surface waves on water of infinite depth. Preprint.

[4] Buffoni, B. 2005 Conditional energetic stability of gravity solitary waves in the presence of weak surface tension. Topol. Meth. Nonlinear Anal. 25, 41-68.

[5] Buffoni, B. 2009 Gravity solitary waves by minimization: an uncountable family. Topol. Meth. Nonlinear Anal. 34, 339-352.

[6] Devaney, R. L. 1976 Reversible diffeomorphisms and flows. Trans. Amer. Math. Soc. 218, 89-113.

[7] Dias, F. \& Iooss, G. 1993 Capillary-gravity solitary waves with damped oscillations. Physica D 65, 399-423.

[8] Djordjevic, V. D. \& Redekopp, L. G. 1977 On two-dimensional packets of capillarygravity waves. J. Fluid Mech. 79, 703-714.

[9] Drazin, P. G. 1983 Solitons. London Mathematical Society Lecture Note Series 85. Cambridge: Cambridge University Press.

[10] Godey, C. 2014 A simple criterion for transverse linear instability of nonlinear waves. Preprint.

[11] Groves, M. D. 2004 Steady water waves. J. Nonlinear Math. Phys. 11, 435-460.

[12] Groves, M. D. \& Haragus, M. 2003 A bifurcation theory for three-dimensional oblique travelling gravity-capillary water waves. J. Nonlinear Sci. 13, 397-447.

[13] Groves, M. D., Haragus, M. \& Sun, S.-M. 2001 Transverse instability of gravitycapillary line solitary water waves. C. R. Acad. Sci. Paris, Sér. 1 333, 421-426.

[14] Groves, M. D., Haragus, M. \& Sun, S.-M. 2002 A dimension-breaking phenomenon in the theory of gravity-capillary water waves. Phil. Trans. Roy. Soc. Lond. A 360, 2189 2243.

[15] Groves, M. D. \& Mielke, A. 2001 A spatial dynamics approach to three-dimensional gravity-capillary steady water waves. Proc. Roy. Soc. Edin. A 131, 83-136.

[16] Groves, M. D. \& WAhlén, E. 2010 On the existence and conditional energetic stability of solitary water waves with weak surface tension. C. R. Math. Acad. Sci. Paris 348, 397402. 
[17] Haragus, M. \& KirChG ÄSsner, K. 1995 Breaking the dimension of a steady wave: some examples. In Nonlinear Dynamics and Pattern Formation in the Natural Environment (eds. Doelman, A. \& van Harten, A.). Pitman Res. Notes Math. Ser. 335, 119-129.

[18] Iooss, G. 1999 Gravity and capillary-gravity periodic travelling waves for two superposed fluid layers, one being of infinite depth. J. Math. Fluid Mech. 1, 24-63.

[19] Iooss, G. \& KIRCHGÄSSnER, K. 1990 Bifurcation d'ondes solitaires en présence d'une faible tension superficielle. C. R. Acad. Sci. Paris, Sér. 1 311, 265-268.

[20] Iooss, G. \& PÉrouème, M. C. 1993 Perturbed homoclinic solutions in reversible 1:1 resonance vector fields. J. Diff. Eqns. 102, 62-88.

[21] Kato, T. 1976 Perturbation Theory for Linear Operators, 2nd edn. New York: SpringerVerlag.

[22] KIRCHGÄSSNER, K. 1982 Wave solutions of reversible systems and applications. J. Diff. Eqns. 45, 113-127.

[23] KIRCHGÄSSNER, K. 1988 Nonlinearly resonant surface waves and homoclinic bifurcation. Adv. Appl. Mech. 26, 135-181.

[24] Milewski, P. A. \& WANG, Z. 2014 Transversally periodic solitary gravity-capillary waves. Proc. Roy. Soc. Lond. A 470, 20130537.

[25] Pego, R. L. \& Sun, S.-M. 2004 On the transverse linear instability of solitary water waves with large surface tension. Proc. Roy. Soc. Edin. A 134, 733-752.

[26] Rousset, F. \& Tzvetkov, N. 2011 Transverse instability of the line solitary waterwaves. Invent. Math. 184, 257-388.

[27] Sulem, C. \& Sulem, P. L. 1999 The Nonlinear Schrödinger Equation. Applied Mathematical Sciences 139. New York: Springer-Verlag.

[28] TAJiRI, M. \& Murakami, Y. 1990 The periodic solution resonance: solutions of the Kadomtsev-Petviashvili equation with positive dispersion. Phys. Lett. A 143, 217-220. 